Aus der Klinik für Kardiologie und Pneumologie

(Prof. Dr. med. G. Hasenfuß)

der Medizinischen Fakultät der Universität Göttingen

\title{
Einfluss des obstruktiven Schlafapnoesyndroms auf die Langzeitprognose von Patienten mit kardiovaskulären Risikofaktoren
}

\author{
INAUGURAL-DISSERTATION \\ zur Erlangung des Doktorgrades \\ der Medizinischen Fakultät der \\ Georg-August-Universität Göttingen
}

vorgelegt von

Jennifer Koch, geb. Labes

aus

Witzenhausen

Göttingen 2018 
Dekan:

Prof. Dr. rer. nat. H. K. Kroemer

Referent:

Prof. Dr. med. R. Wachter

Ko-Referent:

PD Dr. med. I. F. Brandes

Datum der mündlichen Prüfung: $\quad$ 04.12.2018 
Hiermit erkläre ich, die Dissertation mit dem Titel „Einfluss des obstruktiven Schlafapnoesyndroms auf die Langzeitprognose von Patienten mit kardiovaskulären Risikofaktoren“ eigenständig angefertigt und keine anderen als die von mir angegebenen Quellen und Hilfsmittel verwendet zu haben.

Göttingen, den

(Unterschrift) 


\section{Inhaltsverzeichnis}

Abbildungsverzeichnis.......................................................................................................................

Tabellenverzeichnis.................................................................................................................................

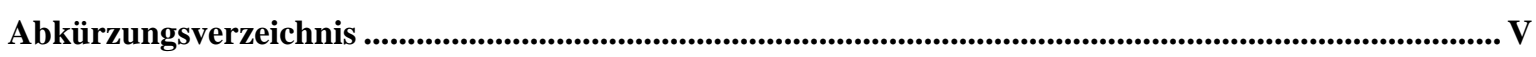

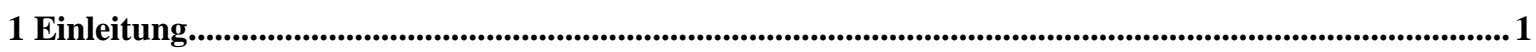

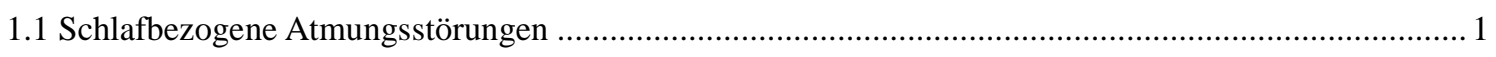

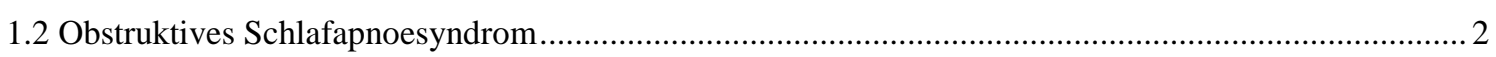

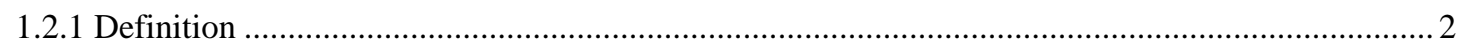

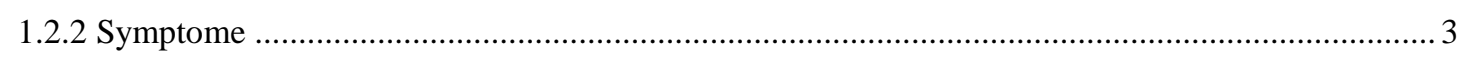

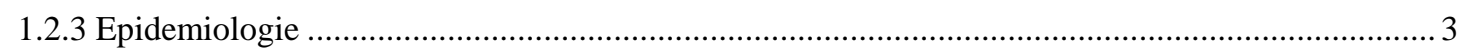

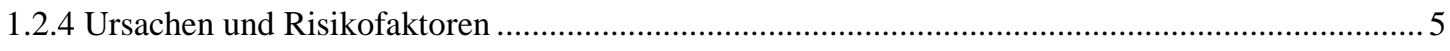

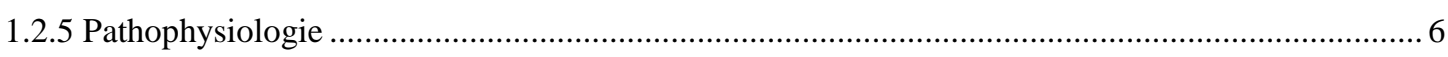

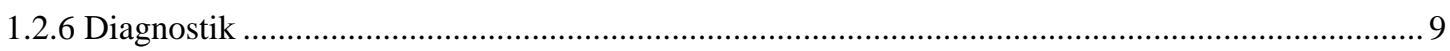

1.2 .7 Therapie

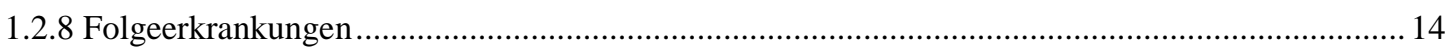

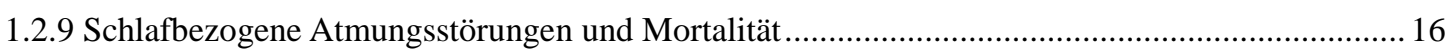

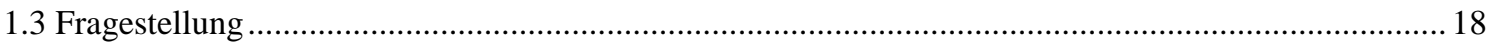

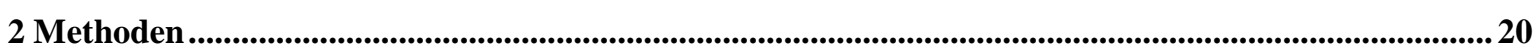

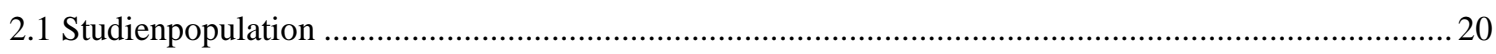

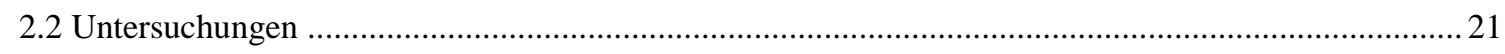

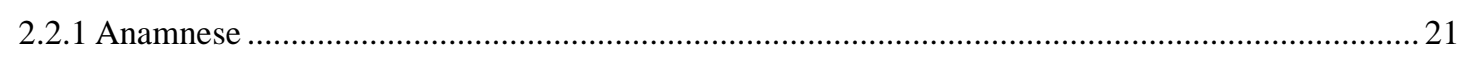

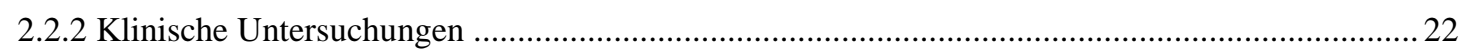

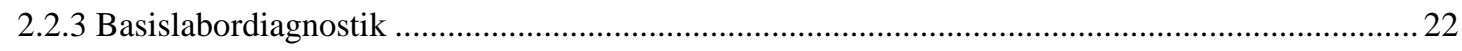

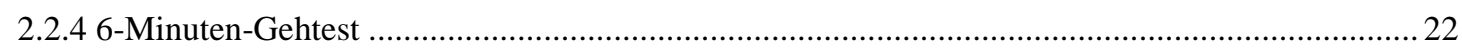

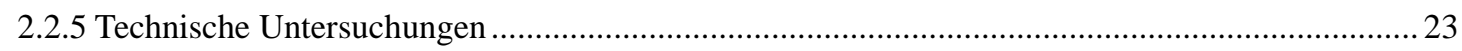

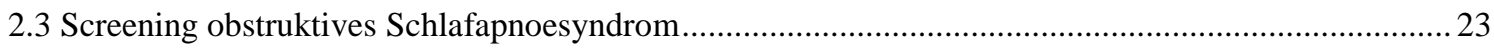

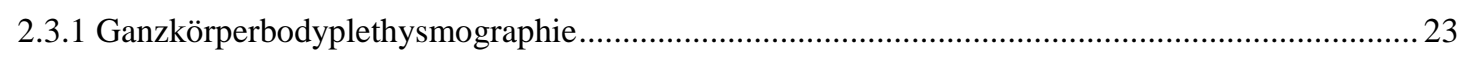

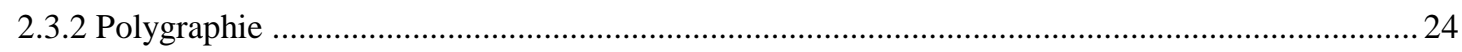

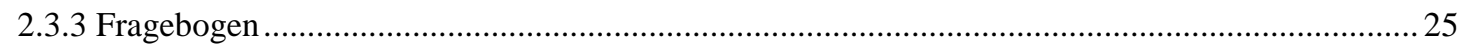

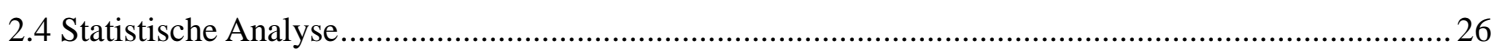

3 Ergebnisse.................................................................................................................................................... 28

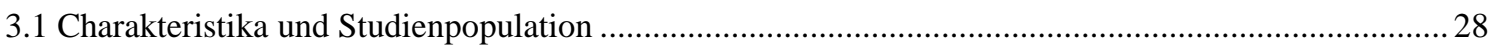

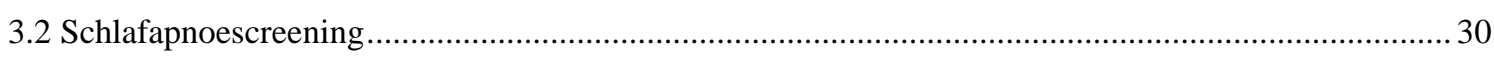

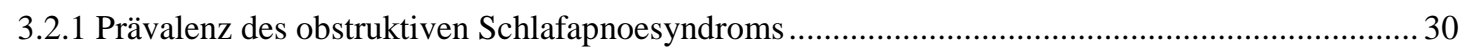

3.2.2 Vergleich des Screeningkollektivs nach AHI ………............................................................. 31

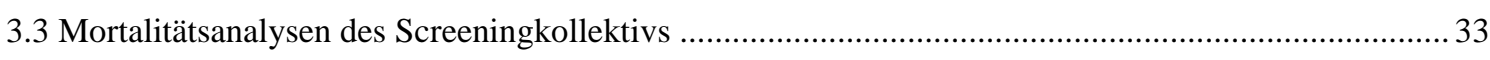

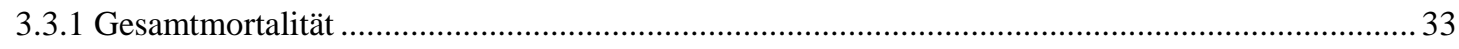

3.3.2 Vergleich und Einfluss des Geschlechts auf die Überlebenszeit.................................................... 36

3.3.3 Einfluss der Variablen Geschlecht und Alter auf die Überlebenszeit ............................................... 37 
3.4 Inzidenzen von kardiovaskulären Ereignissen (MACE) .38

3.5 Prognostischer Einfluss von polygraphisch messbaren Parametern ................................................ 43

3.5.1 Mittlere Sauerstoffsättigung 44

3.5.2 Kumulative Entsättigungszeit, Sauerstoffentsättigungsindex und minimale Sauerstoffsättigung . 46

4 Diskussion 47

4.1 Prävalenz des OSAS im Vergleich mit anderen Studien 47

4.2 Gesamtmortalität des Screeningkollektivs im Vergleich mit anderen Studien ................................55

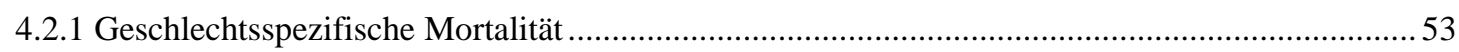

4.2.2 Einfluss des Alters auf den AHI und die Mortalität............................................................... 54

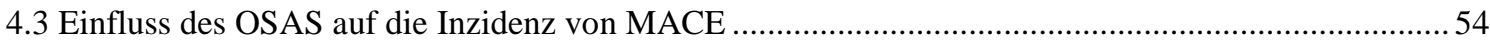

4.4 Evaluation eines prognostischen Parameters für die Mortalität .....................................................5 57

4.4.1 Einfluss der schlafbezogenen Hypoxie auf die Mortalität..........................................................5 57

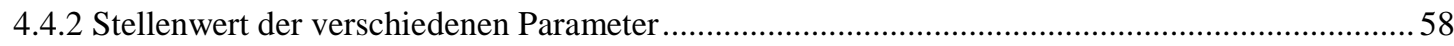

4.4.3 Einfluss der mittleren Sauerstoffsättigung und kumulativen Entsättigungszeit auf die Mortalität 59

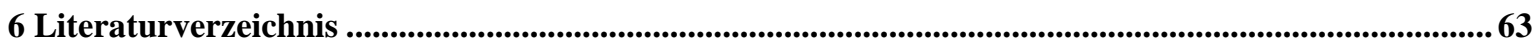




\section{Abbildungsverzeichnis}

Abbildung 1: Schematische Darstellung der Atemwege bei einer obstruktiven

Hypopnoe und Apnoe im Vergleich zu freien Atemwegen

Abbildung 2: Unterscheidung der verschiedenen Apnoeformen anhand der

Polysomnographie

Abbildung 3: Polygraphie-Messgerät am Patienten

Abbildung 4: Kaplan-Meier-Kurven für das Screeningkollektiv nach

Schlafapnoestadium

Abbildung 5: Kaplan-Meier-Kurven für das Screeningkollektiv nach

Geschlecht.

Abbildung 6: Inzidenz von Todesfällen und MACE für das Screeningkollektiv nach AHI als Balkendiagramm dargestellt 38

Abbildung 7: Inverse Kaplan-Meier-Kurven für die Inzidenz von MACEs für das Screeningkollektiv nach Schlafapnoestadium.

Abbildung 8: Inverse Kaplan-Meier-Kurven von kumulativen Inzidenzen der einzelnen MACE für Studienteilnehmer mit und ohne OSAS.

Abbildung 9: Kaplan-Meier-Kurven für das Screeningkollektiv nach mittlerer Sauerstoffsättigung. 45 


\section{Tabellenverzeichnis}

Tabelle 1: Klassifikation der schlafbezogenen Atmungsstörungen .................................. 1

Tabelle 2: Klinische Charakteristika der Studienteilnehmer mit und ohne Schlafapnoescreening

Tabelle 3: Prävalenz für das OSAS des Screeningkollektivs $(n=378)$ in vier Gruppen nach AHI eingeteilt, getrennt nach Geschlecht

Tabelle 4: Klinische Charakteristika des Screeningkollektivs nach Schweregrad des OSAS.

Tabelle 5: Anzahl der verstorbenen Patienten nach AHI und getrennt nach Geschlecht

Tabelle 6: Cox-Regressionsanalyse, adjustiert auf Alter und Geschlecht 34

Tabelle 7: Ergebnisse der Cox-Regression für die Überlebenszeit, adjustiert auf Geschlecht

Tabelle 8: Ergebnisse der Cox-Regression für die Überlebenszeit, adjustiert auf Alter und Geschlecht

Tabelle 9: Inzidenzen der einzelnen MACE des Screeningkollektivs sowohl nach AHI getrennt als auch innerhalb des Gesamtkollektivs dargestellt

Tabelle 10: Cox-Regressionsanalyse für MACE, adjustiert auf Alter und Geschlecht

Tabelle 11: Anzahl der Patienten mit validem bzw. fehlerhaftem Messergebnis sowie $25 \%$ - und $75 \%$ - Perzentile und Median der gemessenen Parameter

Tabelle 12: Vergleich der mittleren Sauerstoffsättigung der Teilnehmer nach Perzentile; je Anzahl und Todesfälle.

Tabelle 13: Vergleich der Ergebnisse der t90\%, des ODI und der minimalen Sauerstoffsättigung der Teilnehmer nach Perzentile; je Anzahl und Todesfälle 


\section{Abkürzungsverzeichnis}

AASM

Abb.

AHI

BMI

CPAP

CRP

EEG

EKG

EMG

ESS

HDL

$\mathrm{HNO}$

HR

IQR

KHK

LDL

MACE

$\mathrm{mmHg}$

NREM

NT - proBNP

ODI

OSAS

$\mathrm{O}_{2}$

pAVK

REM

SBAS

SHHS

TIA

TSH

t90\%

vs.
American Academy of Sleep Medicine

Abbildung

Apnoe-Hypopnoe-Index

Body-Mass-Index

continuous positive airway pressure

C-reaktives Protein

Elektroenzephalogramm

Elektrokardiogramm

Elektromyogramm

Epworth-Schläfrigkeitsskala

high-density lipoprotein

Hals-Nasen-Ohren-Heilkunde

Hazard Ratio

interquartile range

koronare Herzkrankheit

low-density lipoprotein

major adverse cardiac event

Millimeter Quecksilbersäule

non rapid eye movement

$N$-terminal pro brain natriuretic peptide

oxygen desaturation index

obstruktives Schlafapnoesyndrom

chemische Formel für Sauerstoff

periphere arterielle Verschlusskrankheit

rapid eye movement

schlafbezogene Atmungsstörungen

Sleep Heart Health Study

transitorische ischämische Attacke

Thyreoidea-stimulierendes Hormon

kumulative Entsättigungszeit mit Sauerstoffsättigungen $<90 \%$

versus 


\section{Einleitung}

\subsection{Schlafbezogene Atmungsstörungen}

Schlafbezogene Atmungsstörungen (im Folgenden als SBAS abgekürzt) und die daraus resultierenden Erkrankungen sind aufgrund der steigenden Inzidenz und des sozioökonomischen Stellenwertes in der westlichen Bevölkerung in den letzten Jahren zunehmend in den Fokus der Wissenschaft gerückt (Lam et al. 2010; Mannarino et al. 2012). SBAS können zu beträchtlichen Störungen der Schlafqualität führen, was eine ausgeprägte Tagesmüdigkeit, erhöhte Unfallrate und reduzierte Lebensqualität nach sich ziehen kann.

Die schlafbezogenen Atmungsstörungen, die den intrinsischen Dyssomnien zugeordnet werden, werden in zwei Gruppen eingeteilt. Man unterscheidet die schlafbezogenen Atmungsstörungen mit Obstruktion der oberen Atemwege von solchen ohne das Vorliegen einer Obstruktion. Der Anteil der SBAS mit Obstruktion machen den deutlich größeren Anteil mit > $90 \%$ aus (Herold 2016). In der folgenden Tabelle sind diese zwei Gruppen mit den jeweilig unterschiedenen Krankheitsbildern aufgelistet.

Tabelle 1: Klassifikation der schlafbezogenen Atmungsstörungen, Einteilung modifiziert nach Hader et al. 2004 und Herold 2016

\begin{tabular}{|c|c|}
\hline $\begin{array}{c}\text { SBAS mit Obstruktion der oberen } \\
\text { Atemwege }\end{array}$ & $\begin{array}{c}\text { SBAS ohne Obstruktion der oberen } \\
\text { Atemwege }\end{array}$ \\
\hline $\begin{array}{l}\text { - Obstruktives Schnarchen } \\
\text { - Obstruktives Schlafapnoesyndrom } \\
\text { - Upper airway resistance syndrome }\end{array}$ & $\begin{array}{l}\text { - Zentrales Schlafapnoesyndrom und } \\
\text { Cheyne-Stokes-Atmung } \\
\text { - } \quad \text { primäre alveoläre Hypoventilation } \\
\text { - } \quad \text { sekundäre alveoläre Hypoventilation }\end{array}$ \\
\hline
\end{tabular}

In den letzten Jahren hat insbesondere die Erkennung und Behandlung des obstruktiven Schlafapnoesyndroms, das die häufigste Form der SBAS darstellt, an Bedeutung gewonnen. Obwohl das obstruktive Schlafapnoesyndrom (im Weiteren als OSAS abgekürzt) eine verbreitete Schlafstörung ist und die diagnostischen Möglichkeiten zuletzt große Fortschritte gemacht haben, bleibt es jedoch oftmals unerkannt (Mannarino et al. 2012).

Es gibt zunehmend überzeugende Daten, dass das OSAS als eigenständiger Risikofaktor für die Entstehung von kardiovaskulären und neurokognitiven Erkrankungen sowie psychi- 
schen Störungen angesehen werden kann (Malhotra et al. 2015). Zudem gibt es Hinweise, dass die unbehandelte Erkrankung mit einer gesteigerten kardio- und zerebrovaskulären Morbidität und Mortalität assoziiert ist (Lam et al. 2010; Punjabi 2008; Redline 2017; Young et al. 2008). Daher ist die frühzeitige Diagnose des Schlafapnoesyndroms entscheidend, um durch adäquate Therapie lebensbedrohliche Folgeerkrankungen zu vermeiden (Mannarino et al. 2012).

Die vorliegende Arbeit befasst sich mit dem Einfluss des OSAS auf die Langzeitprognose von Patienten mit kardiovaskulären Risikofaktoren.

In den folgenden Abschnitten soll zunächst das OSAS näher dargestellt werden.

\subsection{Obstruktives Schlafapnoesyndrom}

\subsubsection{Definition}

Das OSAS ist durch wiederholte Obstruktionen der oberen Atemwege charakterisiert, die zu Sauerstoffentsättigungen und Aufwachreaktionen führen (American Academy of Sleep Medicine Task Force 1999). Die Ursache ist ein Kollaps der Pharynxmuskulatur während des Schlafs mit kompletter oder teilweiser Obstruktion der oberen Atemwege (Lam et al. 2010; Mannarino et al. 2012). Folge der Obstruktion ist ein reduzierter oder komplett sistierter inspiratorischer Atemfluss (im Folgenden als Hypopnoe oder Apnoe definiert) bei fortbestehenden Atemexkursionen des Thorax, der eine insuffiziente alveoläre Ventilation bedingt. Diese Hypoventilation führt wiederum zu Sauerstoffentsättigungen sowie einem sukzessiven Anstieg des $\mathrm{PaCO}_{2}{ }^{1}$ und resultiert schließlich in einer als Arousal bezeichneten Aufwachreaktion (American Academy of Sleep Medicine Task Force 1999; Hader et al. 2004).

Der Schweregrad des OSAS wird durch die Anzahl an Apnoen und Hypopnoen pro Stunde Schlaf bestimmt und wird als sogenannter Apnoe-Hypopnoe-Index (im Folgenden als AHI abgekürzt) bezeichnet.

Während dem obstruktiven Schlafapnoesyndrom eine mechanische Ursache zugrunde liegt, ist das zentrale Schlafapnoesyndrom dagegen durch einen aufgehobenen zentralen Atemantrieb bedingt.

\footnotetext{
${ }^{1} \mathrm{PaCO}_{2}=$ arterieller Kohlenstoffdioxid-Partialdruck
} 


\subsubsection{Symptome}

Als Leitsymptom des OSAS findet sich ein lautes und unregelmäßiges Schnarchen mit Atemstillständen, das meist vom Partner wahrgenommen wird (Andreas 2012). Daneben ist das OSAS durch wiederholtes Erwachen, gestörten Schlaf und konsekutive ausgeprägte Tagesmüdigkeit mit Einschlafneigung bei monotonen Tätigkeiten charakterisiert (Jordan et al. 2014).

Als dramatische Folge führt die Tagesschläfrigkeit bei Patienten mit OSAS zu einem erhöhten Unfallrisiko (Andreas 2012; Douglas und Polo 1994; Lam et al. 2010; Young et al. 2002a).

Weitere häufige Symptome des OSAS sind in der folgenden Tabelle zusammengefasst und werden nach Tages- und Nachtsymptomatik unterschieden.

Tabelle 1: Symptome des obstruktiven Schlafapnoesyndroms (modifiziert nach Andreas 2012; Bradley und Floras 2003; Herold 2016; Mannarino et al. 2012)

\begin{tabular}{|c|c|}
\hline Nächtliche Symptomatik & Tagessymptomatik \\
\hline $\begin{array}{l}\text { - } \text { Apnoen } \\
\text { - } \text { Lautes, unregelmäßiges Schnarchen } \\
\text { - Unterbrochener, nichterholsamer } \\
\text { - Schlaf } \\
\text { - } \text { Durst } \\
\text { - Nykturie } \\
\text { - Nachtschweiß } \\
\text { - Gastro-ösophagealer Reflux } \\
\text { - Erhöhter Speichelfluss } \\
\text { - Libido- und Potenzstörungen }\end{array}$ & $\begin{array}{l}\text { - } \text { Ausgeprägte Tagesschläfrigkeit } \\
\text { - } \text { Fatigue } \\
\text { - } \text { Morgendliche Mundtrockenheit } \\
\text { - } \text { Morgendliche Kopfschmerzen } \\
\text { - Konzentrationsstörungen } \\
\text { - } \text { Depressive Verstimmung }\end{array}$ \\
\hline
\end{tabular}

\subsubsection{Epidemiologie}

Das obstruktive Schlafapnoesyndrom ist ein verbreitetes Krankheitsbild und stellt die häufigste Form der schlafbezogenen Atmungsstörungen dar (Herold 2016).

Multiple Faktoren führen jedoch dazu, dass das OSAS insgesamt unterdiagnostiziert ist und die Prävalenz damit deutlich unterschätzt wird (Punjabi 2008; Young et al. 2004). Hier spielen beispielsweise Probleme der Infrastruktur (mangelnde Verfügbarkeit der Schlafla- 
bore) eine Rolle. Daneben ist die primäre Krankheitserkennung durch mangelnde Sensibilisierung für das Krankheitsbild eingeschränkt, wenn eine typische Symptomatik wie Schnarchen oder Tagesmüdigkeit nicht vorhanden ist oder nicht beklagt wird (Young et al. 2002a).

Die Prävalenz des OSAS mit einem AHI $\geq 5 / \mathrm{h}$ und Symptomatik der Tagesschläfrigkeit beträgt im Mittel 3 bis $7 \%$ der erwachsenen Männer und 2 bis $5 \%$ der Frauen (Punjabi 2008; Young et al. 1993). Young et al. (1993), die ein US-amerikanisches Kollektiv im Alter von 30 bis 60 Jahren in einer großen Querschnittsstudie (die sogenannte Wisconsin Cohort Sleep Study) untersuchten, fanden bei $9 \%$ der Frauen und $24 \%$ der Männer einen AHI $\geq 5 / h$. Die Prävalenz des asymptomatischen OSAS liegt schätzungsweise bei 20 bis $30 \%$ der Bevölkerung (Young et al. 2004). Eine aktuellere Arbeit von Peppard zeigte, dass die Prävalenz der Schlafapnoe (AHI $\geq 15 / \mathrm{h}$ bei $13 \%$ der Männer und $6 \%$ der Frauen gegenüber $9 \%$ und $4 \%$ von 1994) in den vergangenen zwei Dekaden bei Personen mittleren Alters bereits deutlich zugenommen hat (Peppard et al. 2013).

Männer erkranken häufiger an einem OSAS als Frauen. In der Literatur wird eine zwei- bis dreifach erhöhte Prävalenz des OSAS für das männliche Geschlecht angegeben (Malhotra et al. 2015; Young et al. 1993). Die Häufigkeit des OSAS für das weibliche Geschlecht wird oftmals unterschätzt, da Frauen eher über eine unspezifische Klinik wie Erschöpfung berichten und somit nicht diagnostiziert werden (Punjabi 2008).

Die Prävalenz des OSAS steigt mit dem Alter; in der Gruppe der über 65-jährigen fand sich eine etwa zwei- bis dreifach erhöhte Prävalenz (Bixler et al. 1998; Young et al. 2004). Ähnliche Ergebnisse zeigten auch Young et al. (2002b) in der großen multizentrischen Sleep Heart Health Study (SHHS) sowie die Arbeit von Duran an einem spanischen Patientenkollektiv (Duran et al. 2001). Die Häufigkeit des OSAS steigt mit zunehmendem Alter und erreicht im Alter über 65 Jahren ein Plateau (Duran et al. 2001; Young et al. 2002a; Young et al. 2004).

Wie im folgenden Abschnitt gezeigt werden wird, ist Adipositas der stärkste Risikofaktor für die Entwicklung eines OSAS. Mit einer erwarteten Zunahme der Prävalenz der Adipositas geht auch eine ansteigende Häufigkeit des OSAS einher (Malhotra et al. 2015; Mannarino et al. 2012; Peppard et al. 2013; Schwartz et al. 2008).

Ferner führen Verbesserungen in der technischen Diagnostik und das Älterwerden der Bevölkerung zu einer ansteigenden Prävalenz (Malhotra et al. 2015). 
In diesem Abschnitt wurde die Epidemiologie des OSAS dargestellt; im folgenden Abschnitt soll näher auf die Ursachen und Risikofaktoren des OSAS eingegangen werden.

\subsubsection{Ursachen und Risikofaktoren}

Die Ursachen, die zu einem Kollaps der Pharynxmuskulatur führen und somit die Entstehung eines OSAS begünstigen, sind multifaktoriell. Im Vordergrund stehen morphologische (Adipositas, Verengungen des pharyngealen Raums), demographische (männliches Geschlecht, höheres Alter, genetische Prädisposition), metabolische und endokrine Faktoren (Hypothyreose, Akromegalie, Menopause). Ferner inhibieren Alkoholkonsum, Nikotinabusus und Sedativaeinnahme die Funktion der dilatierend wirkenden Pharynxmuskulatur (American Academy of Sleep Medicine Task Force 1999; Lam et al. 2010; Punjabi 2008).

Adipositas ist der bedeutendste Risikofaktor für die Entstehung eines OSAS (Bradley und Floras 2003; Punjabi 2008; Young et al. 2002a). Peppard et al. zeigten, dass eine Gewichtsänderung von $10 \%$ einen Anstieg bzw. eine Reduzierung des AHI zur Folge hat. Durch Gewichtszunahme steigt das Risiko, ein OSAS zu entwickeln, um ein Sechsfaches an (Peppard et al. 2000b). Die durch Übergewicht bedingte erhöhte pharyngeale Fettgewebsanlagerung verengt die oberen Atemwege, wodurch die Kollapsneigung zunimmt (Douglas und Polo 1994; Eckert und Malhotra 2008; Mannarino et al. 2012; Schwartz et al. 2008). Durch die abdominellen Fettgewebsanlagerungen entsteht eine Reduzierung der funktionellen Residualkapazität, die zu einer Verminderung des Lungenvolumens sowie einem erhöhtem Sauerstoffbedarf des Körpers führen kann (Eckert und Malhotra 2008; Lam et al. 2010; Young et al. 2002a). Ferner ist Adipositas mit einer funktionellen Beeinträchtigung der oberen Atemwegsmuskulatur assoziiert (Carrera et al. 2004).

Weiterhin zeigten epidemiologische Studien, dass das männliche Geschlecht als ein Risikofaktor für das OSAS gilt. In allen untersuchten Altersklassen haben Männer im Vergleich zu Frauen ein zwei- bis dreifach erhöhtes Risiko, an einem Schlafapnoesyndrom zu erkranken (Punjabi 2008; Young et al. 1993). Eine mögliche Erklärung könnte die unterschiedliche Verteilung des Körperfettgewebes sein, das unter anderem hormonell bedingt ist (Lam et al. 2010). Männer entwickeln in der Regel mehr Fettgewebe um den Hals und die pharyngealen Atemwege herum sowie am Thorax und Abdomen, das wiederum zu der oben beschrieben Funktionseinschränkung der Atmung führt. 
Der hormonelle Einfluss auf die Entwicklung eines OSAS zeigt sich auch darin, dass die Prävalenz bei postmenopausalen Frauen gegenüber prämenopausalen Frauen höher ist (Young et al. 2004). Bixler et al. zeigten, dass postmenopausale Frauen mit Hormonersatztherapie kein erhöhtes Risiko für die Entstehung eines OSAS aufwiesen, wohingegen bei Frauen ohne Hormonersatztherapie ein vierfach erhöhtes Risiko für ein OSAS gegenüber prämenopausalen Frauen zu beobachten war (Bixler et al. 2001).

Auch anatomische Veränderungen wie kraniofaziale Fehlbildungen können eine Verengung der oberen Atemwege begünstigen und die Kollapsneigung während des Schlafs erhöhen. Dazu sind beispielsweise Retrognathie, Makroglossie, Tonsillenhyperplasie sowie maxilläre oder mandibuläre Retroposition zu zählen (Lam et al. 2010; Mannarino et al. 2012; Punjabi 2008).

\subsubsection{Pathophysiologie}

Wie bereits im Kapitel Ursachen und Risikofaktoren beschrieben, ist die Hauptursache des OSAS ein Kollaps der Pharynxmuskulatur mit folgender Obstruktion während der Inspiration (Bradley und Floras 2003; Deegan und McNicholas 1995).

Während der Inspiration entsteht durch die Ausdehnung des Thorax ein Unterdruck in den Atemwegen. Der pharyngeale Abschnitt der Atemwege dient als kollapsibiles Segment der Atemwege, da er nur durch die Aktivität der Pharynxmuskulatur offen gehalten werden kann. Falls dieses pharyngeale Segment während der Inspiration nicht mehr ausreichend offen gehalten werden kann, erhöht sich der Atemwegswiderstand und es entstehen Vibrationen (Schnarchen) oder zunehmende Verengung des Atemweges bis hin zur obstruktiven Apnoe (vgl. Abbildung 1) (Bradley und Floras 2003; Stuck und Maurer 2013). 


\section{Freie Atemwege}

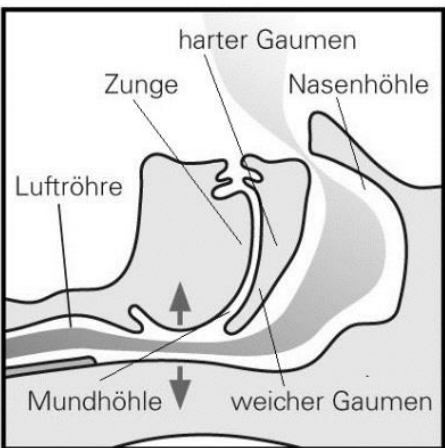

Atemfluss

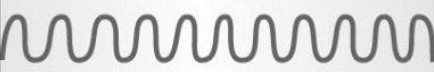

Normale Atmung
Verengte Atemwege bei

einer obstruktiven Hypopnoe
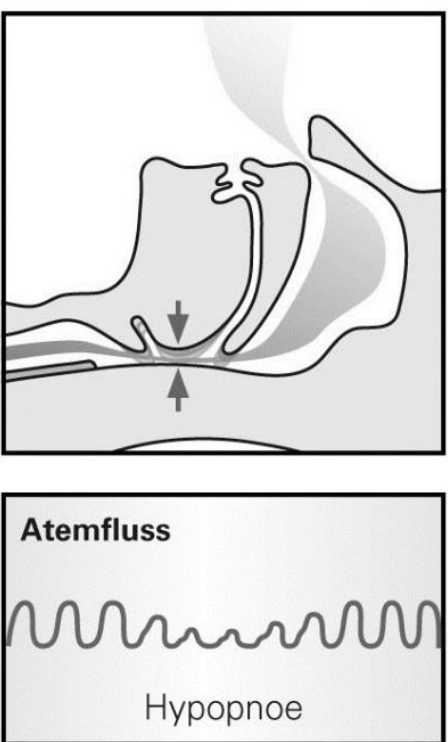

Verschlossene Atemwege

bei einer obstruktiven Apnoe

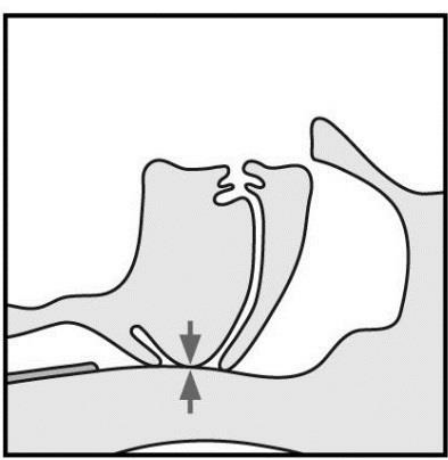

Atemfluss

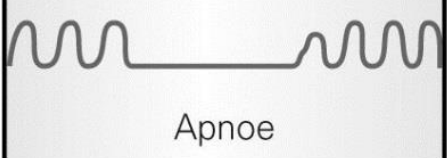

Abbildung 1: Schematische Darstellung der Atemwege bei einer obstruktiven Hypopnoe und Apnoe im Vergleich zu freien Atemwegen (modifiziert nach https://www.resmedshop.de/themenwelt/schlafapnoe 2017)

Als unmittelbare Folge der Obstruktion treten eine erhöhte Atemarbeit, eine Hypoxämie, seltener auch eine Hyperkapnie und intrathorakale Druckschwankungen auf (Quack et al. 2010). Durch die erhöhte Atemarbeit werden über Chemorezeptorstimulation Arousals hervorgerufen, die zu einer Unterbrechung der Schlafstruktur führen. Durch die Arousals wird die Aktivität und Empfindlichkeit der erschlafften pharyngealen Dilatatormuskeln der oberen Atemwege gesteigert und es kommt zu einer Wiederöffnung der Atemwege. Der Patient wacht auf (Schnarchgeräusch), hyperventiliert zur Kompensation der Hypoxämie und Hyperkapnie und schläft wieder ein. Im Folgenden sinken die Chemosensitivität, das Lungenvolumen und die Aktivität der Dilatatormuskeln (Reduzierung der Atemarbeit). Es folgt eine Hypoventilation und schließlich resultiert erneut ein Kollaps der Pharynxmuskulatur mit den damit verbundenen obstruktiven Apnoen/Hypopnoen. Dieser Circulus vitiosus wird einige hundert Mal pro Nacht wiederholt. Die Schlafunterbrechungen behindern das Erreichen der Tiefschlafphasen (Eckert und Malhotra 2008).

Im Schlaf kommt es zu einer physiologischen kardiovaskulären Relaxation. Während des NREM-Schlafs, der ca. $85 \%$ der Schlafzeit ausmacht, sinken der Blutdruck und die Herzfrequenz durch die erhöhte Vagusaktivität und den verminderten Sympathikotonus ab (Brisco und Goldberg 2010). Durch die nächtlichen Apnoen wird dieser physiologische Ablauf unterbrochen (Kasai 2012). 
Die obstruktiven Apnoen rufen eine Reihe von mechanischen, hämodynamischen, chemischen, neuralen und inflammatorischen Prozessen hervor, die zur Entwicklung von kardiovaskulären Erkrankungen beitragen und in den folgenden Abschnitten näher betrachtet werden (Bradley und Floras 2003; McNicholas et al. 2007; Meier und Andreas 2012).

- Mechanische und hämodynamische Effekte

Die Atemarbeit gegen den verengten Pharynx verursacht einen erhöhten negativen intrathorakalen Druck (Bradley und Floras 2003; Hader et al. 2004; Siegenthaler und Blum 2006). Dadurch wird der linksventrikuläre transmurale Druck und infolgedessen die Nachlast erhöht (Bradley und Floras 2009). Gleichzeitig ist auch der venöse Rückstrom zum rechten Ventrikel erhöht, was letztendlich die diastolische linksventrikuläre Füllung behindert (durch vermehrte Dehnung des rechten Ventrikels und linksseitige Verschiebung des interventrikulären Septums). Das Zusammenspiel aus erniedrigter linksventrikulärer Vorlast und erhöhter linksventrikulärer Nachlast reduziert das Schlagvolumen sowie die Herzleistung und begünstigt somit die Entstehung einer Herzinsuffizienz (Bradley und Floras 2003).

- Neurohumorale Effekte

Obstruktive Schlafapnoen führen zu einer gesteigerten Aktivität des sympathischen Nervensystems und zu einer Abnahme des Vagotonus. Hypoxämie, Hyperkapnie und Arousals unterstützen diesen Vorgang durch Stimulation von peripheren und zentralen Chemorezeptoren (Hedner et al. 2006; Lüthje und Andreas 2008; O'Connor et al. 2009; Young et al. 2002a). Folgen sind eine Erhöhung des peripheren Widerstandes durch Vasokonstriktion mit Blutdruckanstieg sowie eine gesteigerte Herzfrequenz, die mit einer erhöhten Herzfrequenzvariabilität durch die kardiale sympathische Erregung einhergeht (Bradley und Floras 2009; Kasai und Bradley 2011). Die Effekte der obstruktiven Apnoen auf das kardiovaskuläre System sind jedoch nicht auf den Schlaf begrenzt; auch tagsüber werden eine erhöhte Sympathikusaktivität und Blutdruckerhöhung registriert (Lüthje und Andreas 2008).

Das Zusammenspiel aus erhöhter linksventrikulärer Nachlast und erhöhter Herzfrequenz steigert den Sauerstoffbedarf des Myokards bei apnoebedingtem reduzierten Sauerstoffangebot (Golbin et al. 2008; Steiner et al. 2005). Hieraus resultiert die Prädisposition dieser Patienten für die Entwicklung kardialer Ischämien, Arrhythmien, linksventrikulärer Hypertrophie und Herzinsuffizienz (Bradley und Floras 2009; Brisco und Goldberg 2010). 
- Endotheliale Dysfunktion und inflammatorische Effekte

Die intermittierende Hypoxämie führt zu oxidativem Stress und zur endothelialen Dysfunktion (Kendzerska et al. 2014). Die Beeinträchtigung der endothelialen Funktion tritt bei OSAS-Patienten mit einer Reduktion der stickstoffmonoxidabhängigen Vasodilatation sowie mit erhöhten Endothelinspiegeln (andauernde Vasokonstriktion) auf und verursacht Schäden an den Gefäßwänden (McNicholas et al. 2007). Die endotheliale Dysfunktion beschleunigt die Entstehung von Atherosklerose, stellt eine Vorstufe der arteriellen Hypertonie dar und ist mit einem erhöhten Risiko für ein Auftreten von kardiovaskulären Erkrankungen vergesellschaftet (Lüthje und Andreas 2008). Daneben gibt es zunehmend Beweise, dass die intermittierende Hypoxämie zur Freisetzung proinflammatorischer Mediatoren sowie oxidativem Stress führt und somit eine entscheidende Rolle in der Bildung von Atherosklerose oder arteriellen Thromben spielt. Mehrere Studien wiesen erhöhte Spiegel von Zytokinen, Matrix-Metallproteinasen, Akute-Phase-Proteinen und EndothelAdhäsionsmolekülen im Blut von OSAS-Patienten nach (Lüthje und Andreas 2008). Die Arbeitsgruppe um Shamsuzzaman beobachtete, dass die Erkrankten höhere Plasma-CRPSpiegel aufweisen, die proportional zur Häufigkeit von Apnoen und Hypopnoen sind (Shamsuzzaman et al. 2003).

\subsubsection{Diagnostik}

Am Anfang der Diagnostik steht die ausführliche, standardisierte Anamnese mit Erhebung von Begleiterkrankungen, des Schlaf-/Wachverhaltens und Fragen nach den in Kapitel 1.2.2 dargestellten Symptomen (Hader et al. 2004). Zusätzlich werden psychometrische Tests wie die Epworth-Schläfrigkeitsskala (ESS) verwendet. Zur objektiven Messung der Tagesschläfrigkeit kann ein Multipler Schlaflatenztest durchgeführt werden (McNicholas 2008). Anschließend folgt die klinische Untersuchung, die neben der körperlichen Untersuchung auch noch ergänzende apparative oder laborchemische Untersuchungen beinhalten kann (S3-Leitlinie Schlafstörungen 2009).

Der Goldstandard zur Diagnostik eines OSAS ist die Polysomnographie; ein computergestütztes Analyseverfahren zur kontinuierlichen nächtlichen Registrierung und Analyse zahlreicher biologischer Signale während des Schlafes. Hierzu gehören die Aufzeichnung eines Elektroenzephalogramms (EEG), Elektrookulogramms, Elektromyogramms (EMG), Elektrokardiogramms (EKG) sowie des Atemflusses, der Thorax- und Abdominalexkursionen, Pulsoxymetrie, Schlafposition, Bewegung und Geräusche (McNicholas 2008). Bis- 
her war für das nächtliche Monitoring die Übernachtung in einem Schlaflabor notwendig; inzwischen sind auch ambulante Schlaftests mit mobilen Screeninggeräten (kardiorespiratorische Polygraphie) verfügbar (Jordan et al. 2014). Diese Methode ist häufiger verfügbar und hat ökonomische Vorteile. Als Nachteil ist jedoch anzumerken, dass die optische und akustische Aufzeichnung des Schlafverhaltens fehlt und weder ein EEG noch EMG registriert werden können (Malhotra et al. 2015). Neuere Empfehlungen sehen die Polygraphie an erster diagnostischer Stelle. Nur bei uneindeutiger Befundkonstellation sollte eine stationäre Polysomnographie folgen (Storre 2016).

\subsubsection{Diagnostische Kriterien für das OSAS}

Die Polysomnographie ermöglicht neben der Ermittlung von AHI, Arousals und gestörter Schlafarchitektur auch die Unterscheidung der verschiedenen Apnoeformen.

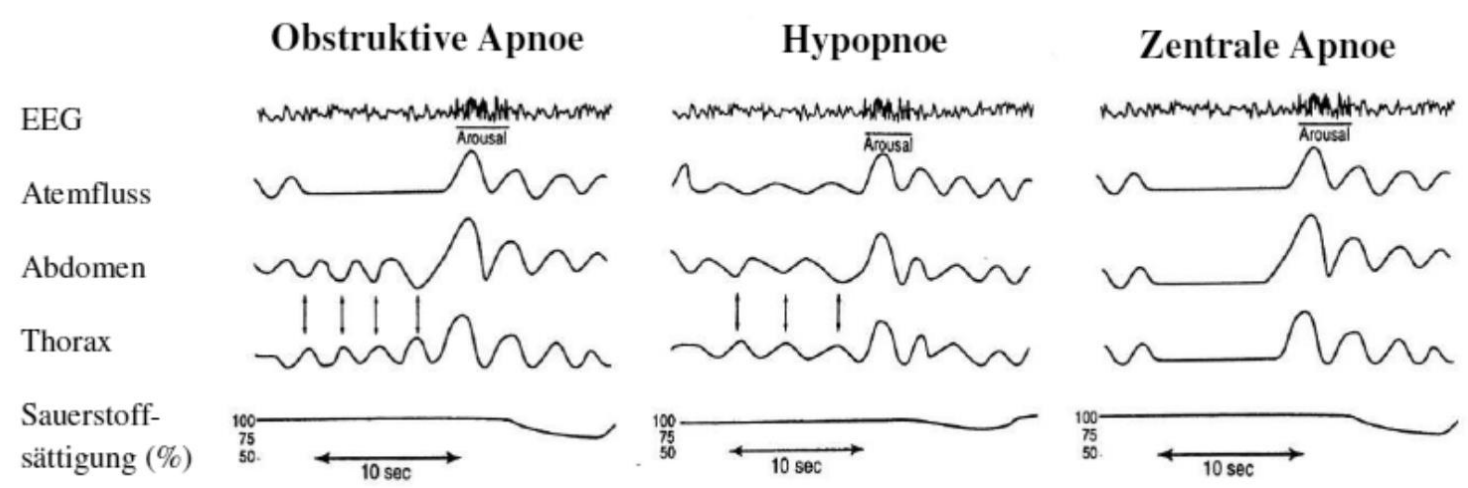

Abbildung 2: Unterscheidung der verschiedenen Apnoeformen anhand der Polysomnographie (modifiziert nach Strollo und Rogers 1996)

Als Apnoen werden Atempausen während des Schlafens mit einer Verminderung des Atemflusses um $\geq 90 \%$ und mit einer Dauer von mindestens 10 Sekunden bezeichnet. Apnoen werden in obstruktive, zentrale oder gemischte Apnoen eingeteilt.

Obstruktive Apnoen sind durch fehlenden Atemfluss bei fortbestehenden inspiratorischen Atmungsanstrengungen und damit verbundenen Thoraxexkursionen gekennzeichnet. Hingegen sind zentrale Apnoen durch eine fehlende Atemarbeit mit einem simultanen Sistieren von Atemexkursionen des Thorax und inspiratorischem Atemfluss definiert (Chowdhury et al. 2010; Rodenbeck 2013).

Hypopnoen sind definiert als Verminderung des Atemflusses um mindestens $50 \%$ für mindestens 10 Sekunden, die mit einem Abfall der Sauerstoffsättigung um $\geq 3 \%$ oder einem Arousal einhergehen (American Academy of Sleep Medicine Task Force 1999). 
Ein Arousal ist eine vorübergehende Aufwachreaktion, die weniger als 10 Sekunden andauert (Bradley und Floras 2003; Lam et al. 2010).

Um den Schweregrad der Schlafapnoe zu ermitteln, wird der AHI bestimmt. Der AHI gibt die Anzahl aller Apnoen und/oder Hypopnoen pro Stunde Schlafzeit an (Bradley und Floras 2003; Herold 2016; Punjabi 2008).

Im Folgenden sind die diagnostischen Kriterien laut der American Academy of Sleep Medicine Task Force (abgekürzt als AASM) von 1999 aufgelistet. Demnach ist die Diagnose eines OSAS gesichert, wenn Kriterium A oder B plus Kriterium C (AHI $\geq 5 / \mathrm{h})$ erfüllt ist oder wenn ein $\mathrm{AHI} \geq 15 / \mathrm{h}$ bei fehlenden Symptomen vorliegt (American Academy of Sleep Medicine Task Force 1999; Herold 2016; Mannarino et al. 2012; McNicholas 2008).

A. Ausgeprägte Tagesschläfrigkeit, die nicht durch andere Faktoren erklärt werden kann

B. Zwei oder mehr der folgenden Punkte, die nicht durch andere Faktoren erklärt werden können

- Würgen oder Keuchen während des Schlafes

- Wiederholtes Aufwachen während des Schlafes

- Nicht erholsamer Schlaf

- Tagesmüdigkeit

- Konzentrationsstörungen

C. $\quad \mathrm{AHI} \geq 5 / \mathrm{h}$

\subsubsection{Schweregradeinteilung des OSAS nach dem AHI}

Das OSAS wird nach dem Report der AASM (1999) auf der Grundlage des nächtlichen Monitorings mit Bestimmung des AHI in drei Schweregrade eingeteilt:

- Mild:

- Moderat:

- Schwer:
AHI $5-14 / h$

AHI $15-29 / \mathrm{h}$

$\mathrm{AHI} \geq 30 / \mathrm{h}$ 


\subsubsection{Therapie}

Der AHI allein ist kein geeigneter Indikator zur Therapieeinleitung. Kontrovers diskutiert wird auch die Therapieindikation bei Patienten mit fehlender Tagesschläfrigkeit (Pack und Maislin 2001; Young et al. 2008). Eine Indikation zur Therapie besteht laut Sanner, wenn das OSAS nach ausführlicher Diagnostik die Beschwerdesymptomatik (vor allem Tagesschläfrigkeit) des Patienten erklärt (Sanner et al. 2004).

Ein wichtiges Kriterium für die Therapie (insbesondere die CPAP-Therapie) eines OSAS ist neben der Verbesserung der Symptomatik insbesondere die Reduzierung der kardiovaskulären Morbidität und Mortalität (Marin et al. 2005). Die Behandlung des OSAS beruht auf einem multidisziplinären Ansatz. Als mögliche Behandlungsmodalitäten kann grundlegend zwischen der konservativen, apparativen oder chirurgischen Therapie unterschieden werden.

- Konservative Therapie

Das Prinzip der konservativen Therapie beruht auf Allgemeinmaßnahmen wie Gewichtsreduktion und Optimierung der Schlafhygiene ${ }^{2}$ und sollte stets Teil des Therapiekonzepts sein. Die bei weitem effektivste Maßnahme ist die Gewichtsreduktion (Meier und Andreas 2012). In mehreren prospektiven Studien konnte gezeigt werden, dass bei übergewichtigen Patienten ein Gewichtsverlust von $10 \%$ eine Reduktion des AHI um bis zu $50 \%$ zur Folge hat (Tuomilehto et al. 2013; Young et al. 2002b). Weitere Lebensstiländerungen wie die Umstellung der Ernährungsgewohnheiten und gesteigerte körperliche Aktivität wirken sich positiv aus. Medikamentöse Ansätze zur Therapie des OSAS waren bisher wenig erfolgreich. Die derzeitige Datenlage lässt den Schluss zu, dass die alleinige Pharmakotherapie nicht empfohlen werden kann (Hein und Magnussen 1998; Mason et al. 2013; S3-Leitlinie Schlafstörungen 2009).

\section{- Apparative Therapie}

$\mathrm{Zu}$ den apparativen Behandlungsoptionen zählen die nasale Beatmungstherapie mit Überdruck sowie intraorale Hilfsmittel wie individuell angepasste UnterkieferProtrusionsschienen.

\footnotetext{
${ }^{2}$ Zur Schlafhygiene zählen unter anderem der Verzicht auf Alkoholkonsum, schwere Mahlzeiten und Sedativaeinnahme vor dem Schlafengehen. Zudem wird empfohlen, einen regelmäßigen Schlafrhythmus mit ausreichenden Schlafphasen einzuhalten und das Schlafen in Rückenlage zu vermeiden (Stuck und Maurer 2013).
} 
Die im Jahr 1981 eingeführte nasale CPAP ${ }^{3}$-Therapie nach Sullivan gilt als der Goldstandard in der Therapie für Patienten mit einem symptomatischen OSAS (Malhotra et al. 2015) und reduziert nachweislich das Auftreten von Symptomen (Becker et al. 2003). Neben einer Abnahme der Tagesmüdigkeit kommt es zu einer Verbesserung der neurokognitiven Funktionen und der Lebensqualität (Sanner et al. 2004; Schulz et al. 2006). Zahlreiche Studien haben gezeigt, dass die suffiziente CPAP-Therapie einen positiven Einfluss auf den Blutdruck, nächtliche Herzrhythmusstörungen, Schlaganfallrisiko sowie linksventrikuläre systolische und diastolische Funktion hat (Arias et al. 2005; Becker et al. 2003; Benjamin und Lewis 2008; Gottlieb et al. 2014; Kasai 2012; Lüthje und Andreas 2008; Oliveira et al. 2009). Zudem führt die CPAP-Therapie zu einer Verminderung von myokardialen Ischämien während des Schlafs (Peled et al. 1999). Als weitere günstige Effekte auf pathophysiologischer Ebene konnten eine verminderte Sympathikusaktivität, Normalisierung der endothelialen Dysfunktion und Reduzierung der proinflammatorischen Faktoren beobachtet werden (Steiner et al. 2005). Nach aktueller Datenlage kann durch die CPAP-Therapie neben den genannten günstigen Effekten jedoch keine signifikante Verbesserung des Überlebens bzw. Verminderung von kardiovaskulären Ereignissen erzielt werden (McEvoy et al. 2016; Naughton und Kee 2017).

Die CPAP-Therapie wird über eine auf das Gesicht des Patienten angepasste Nasenmaske oder eine Nasen-Mund-Maske durchgeführt. Das Ziel ist einen kontinuierlichen positiven Druck in den Atemwegen aufzubauen, um den negativen intrapharyngealen Drücken, die zum Kollaps der oberen Atemwege führen, entgegenzuwirken (Sanner et al. 2004). Durch diese pneumatische Schienung werden die nächtlichen Apnoen, Sauerstoffentsättigungen und Arousals fast vollständig beseitigt, was wiederum zu einem verbesserten Schlafprofil mit vermehrten Tief- und REM-Schlafphasen führt. Trotz technischer Verbesserungen ist die Langzeitakzeptanz der CPAP-Therapie unter den Patienten eingeschränkt (Hoshino et al. 2016; Sommer et al. 2016).

- Chirurgische Therapie

Chirurgische Maßnahmen sind nur noch indiziert, wenn eindeutig behebbare Ursachen wie beispielsweise anatomische Deformitäten vorliegen (Polo et al. 1994). Ein operatives Vorgehen als alternative Therapiestrategie kann notwendig sein, wenn die apparativen Methoden vom Patienten nicht toleriert werden (Sommer et al. 2016). Die operativen Behandlungsoptionen reichen von invasiven bis zu minimal-invasiven Operationsverfahren und

${ }^{3} \mathrm{CPAP}=$ continuous positive airway pressure 
richten sich vor allem nach der Lokalisation und dem Ausmaß der Obstruktion der oberen Atemwege.

Die Tracheotomie war bis zum Jahr 1981 die einzig effektive Therapieoption des OSAS (Young et al. 2004). Die durchschnittliche Erfolgsrate liegt bei 96,2\%. Heutzutage wird sie nur noch als Ultima Ratio gewählt, da mit dem Tracheostoma eine Einbuße an Lebensqualität einhergeht (Leitlinie HNO-spezifische Therapie der obstruktiven Schlafapnoe bei Erwachsenen 2015). Heutzutage gewinnt die Adipositaschirurgie an Bedeutung, da das Übergewicht der Hauptrisiko für ein OSAS darstellt. Polysomnographische Kontrollen nach operativen Maßnahmen wie Magenband oder Magenbypass konnten eine deutliche Reduktion des AHI zeigen (Fritscher et al. 2007).

Weitere aktuelle operative Therapieoptionen je nach Indikation sind in der folgenden Liste zusammengefasst (Herold 2016; Mannarino et al. 2012; Sommer et al. 2016):

- Tonsillektomie

- Uvulopalatopharyngoplastik

- Laser-assistierte Uvuloplastik

- laserchirurgische Zungengrundreduktion mittels interstitieller Radiofrequenztherapie

- nasalchirurgische Eingriffe

- maxillomandibuläre Umstellungsosteotomie

Aufgrund der großen Incompliance der CPAP-Therapie wird neben der operativen Behandlungsmöglichkeit nach weiteren alternativen Therapieoptionen für das OSAS geforscht. In aktuellen Studien wird das Verfahren der Nervus hypoglossus-Stimulation untersucht. Dieser Nerv innerviert die Dilatatormuskeln der oberen Atemwege und eine Stimulation soll einen Kollaps verhindern (Leitlinie HNO-spezifische Therapie der obstruktiven Schlafapnoe bei Erwachsenen 2015; Malhotra et al. 2015).

\subsubsection{Folgeerkrankungen}

In den letzten Jahren zeigten mehrere epidemiologische Studien, dass das OSAS als Risikofaktor für die Entstehung von kardio- und zerebrovaskulären Erkrankungen gilt (Javaheri et al. 2017). Dies gilt auch unabhängig von anderen Einflussgrößen mit einem erhöhten Risiko wie z.B. Alter, Körpergewicht oder metabolische Erkrankungen (Bradley 
und Floras 2009; Kahwash et al. 2011; Malhotra et al. 2015; Marin et al. 2005; McNicholas et al. 2007). Die zugrunde liegenden pathophysiologischen Mechanismen wurden bereits im Kapitel 1.2.5 erläutert.

Das OSAS gilt als ein unabhängiger Risikofaktor für die Entwicklung einer arteriellen Hypertonie (Nieto et al. 2000; Peppard et al. 2000a). Bis zu $60 \%$ der OSAS-Patienten leiden an einer arteriellen Hypertonie (Hedner et al. 2006). Das OSAS ist als eine der häufigsten Ursachen der sekundären arteriellen Hypertonie anzusehen, wobei die fehlende zirkadiane Tag-Nacht-Rhythmik in der Langzeit-Blutdruck-Messung auffällig ist (Schulz et al. 2006). Laut Peppard et al. (2000a) steigt das Risiko für die Entstehung einer arteriellen Hypertonie mit zunehmendem Apnoe-Hypopnoe-Index an. Neben der systemarteriellen ist auch eine erhöhte Prävalenz der pulmonalarteriellen Hypertonie mit etwa 17 bis $42 \%$ aller OSAS-Patienten zu beobachten (Mannarino et al. 2012).

Auch Herzrhythmusstörungen werden gehäuft bei OSAS-Patienten beobachtet und treten in direktem Zusammenhang zu den nächtlichen Apnoen auf (Schulz et al. 2006). Am häufigsten lassen sich zyklische Undulationen der Herzfrequenz (Sinusbradykardie/tachykardie) beobachten; Sinusarreste sowie atrioventrikuläre Blockbilder konnten bei bis zu $10 \%$ der betroffenen Patienten aufgezeichnet werden (Mannarino et al. 2012; Schulz et al. 2006). Diese bradykarden Herzrhythmusstörungen treten am häufigsten als Folge einer Reflexantwort der Apnoen und Hypoxämie auf (Shamsuzzaman et al. 2003). Daneben beschrieben einige Studien eine Assoziation des OSAS und dem Auftreten von Vorhofflimmern. In einer Subanalyse der SHHS wurde eine vierfache Erhöhung für die Prävalenz von Vorhofflimmern bei Patienten mit einem AHI > 30/h nachgewiesen (Benjamin und Lewis 2008; McNicholas et al. 2007).

Ergebnisse der SHHS zeigten zudem einen kausalen Zusammenhang zwischen dem OSAS und der koronaren Herzkrankheit (KHK) (Shahar et al. 2001). Die Prävalenz der KHK bei Patienten mit einem OSAS beträgt 20 bis $30 \%$ und ist im Vergleich zur Normalbevölkerung um den Faktor 2-3 erhöht (Schulz et al. 2006). Unter Patienten mit einer KHK ist die Prävalenz des OSAS mit 30 bis $60 \%$ höher als in der Normalbevölkerung (Lüthje und Andreas 2008). Das unbehandelte OSAS gilt insbesondere bei kardial erkrankten Patienten als negativer Prognoseparameter und geht mit einer erhöhten Mortalität einher (McNicholas et al. 2007; Shamsuzzaman et al. 2003). Die Häufigkeit der nächtlichen STStreckensenkungen vereinbar mit myokardialen Ischämien nimmt mit steigendem Schwe- 
regrad des OSAS zu und ist abhängig vom Maß der Sauerstoffentsättigung (Shamsuzzaman et al. 2003).

5 bis $10 \%$ der OSAS-Patienten leiden an einer echokardiographisch nachgewiesenen systolischen Herzinsuffizienz (Laaban et al. 2002). Die Datenauswertung der SHHS zeigte ein 2,38-fach erhöhtes Risiko für eine Herzinsuffizienz bei Vorhandensein eines OSAS (Shahar et al. 2001). In mehreren epidemiologischen Studien wurde insbesondere ein signifikanter Zusammenhang zwischen einem schweren OSAS und einer reduzierten globalen ventrikulären Funktion gezeigt (Fung et al. 2002; Varol et al. 2010). Bei herzinsuffizienten Patienten mit einer linksventrikulären Ejektionsfraktion von $<40 \%$ wurde die Prävalenz für SBAS zwischen 40 und $70 \%$ angegeben (Schulz et al. 2007); für das OSAS liegt die Prävalenz zwischen 12 und 53 \% (Kasai und Bradley 2011).

In einer weiteren Studie konnte nachgewiesen werden, dass das moderat bis schwere OSAS einen unabhängigen Risikofaktor für die diastolische Dysfunktion darstellt. Die Prävalenz der diastolischen Dysfunktion steigt mit zunehmendem Schweregrad des OSAS an (Wachter et al. 2013).

Verschiedene Publikationen beschreiben eine kausalen Zusammenhang zwischen obstruktiver Schlafapnoe und Apoplex (Mannarino et al. 2012). Dabei steigt die Inzidenz mit zunehmender Schwere des OSAS an (Redline et al. 2010; Yaggi et al. 2005). Bei ca. 5 bis 10 $\%$ der OSAS-Patienten findet sich ein Apoplex oder transitorische ischämische Attacke (TIA) in der Anamnese (Schulz et al. 2006). In der Entstehung von zerebrovaskulären Folgeerkrankungen des OSAS muss von einer multifaktoriellen Genese ausgegangen werden. $\mathrm{Zu}$ einem erhöhten Schlaganfallrisiko tragen sicherlich auch der oben erwähnte Zusammenhang zwischen dem OSAS und Hypertonie bzw. Herzrhythmusstörungen sowie die durch die endotheliale Dysfunktion bedingten atherosklerotischen Veränderungen hirnversorgender Gefäße bei (Schulz et al. 2000).

\subsubsection{Schlafbezogene Atmungsstörungen und Mortalität}

In den vergangenen Jahren ist zunehmend das erhöhte OSAS-assoziierte Mortalitätsrisiko in den Fokus gerückt (Punjabi et al. 2009). Die Ursachen dafür sind heterogen und lassen sich auf mehreren Ebenen erklären.

Wie im Kapitel 1.2.8 beschrieben, begünstigt das OSAS als unabhängiger Risikofaktor direkt oder indirekt die Entstehung einer arteriellen Hypertonie sowie kardio- und zerebro- 
vaskulärer Erkrankungen. Daraus resultieren für das unbehandelte OSAS eine erhöhte Morbidität und Mortalität (Javaheri et al. 2017; Young et al. 2008; Young et al. 2002a).

Mehrere größere Studien (darunter die Wisconsin Sleep Cohort Study und SHHS) haben die Mortalität bei Patienten mit schlafbezogenen Atmungsstörungen untersucht.

Die Arbeitsgruppe um Young wertete die Mortalität auf Grundlage der Wisconsin Sleep Cohort Study (n=1522) über einen Follow-up-Zeitraum von 18 Jahren aus. Die Ergebnisse zeigten, dass das Mortalitätsrisiko unabhängig von Einflussgrößen wie Alter, Geschlecht oder BMI mit zunehmender Schwere der SBAS ansteigt. Sie fanden, dass Patienten mit einer schweren schlafbezogenen Atmungsstörung ohne CPAP-Therapie ein fast vierfach erhöhtes Mortalitätsrisiko aufweisen (Young et al. 2008). Vergleichbare Ergebnisse wurden von Lee et al. (2013) nachgewiesen, die die Mortalität innerhalb eines koreanischen Kollektivs untersuchten.

Auch die Ergebnisse der SHHS, in der insgesamt 6441 Teilnehmer über einen mittleren Zeitraum von 8,2 Jahren beobachtet worden, zeigten, dass SBAS sowohl mit einer erhöhten Gesamtmortalität als auch kardiovaskulären Mortalität einhergehen. Insbesondere bei Männern im Alter zwischen 40 und 70 Jahren mit einem schweren OSAS zeigte sich ein erhöhtes Mortalitätsrisiko (Punjabi et al. 2009).

Eine kürzlich veröffentliche Meta-Analyse von zwölf prospektiven Kohortenstudien beschreibt, dass nur das schwere OSAS signifikant mit einem erhöhten Mortalitätsrisiko assoziiert ist. Als mögliche Erklärung wird angeführt, dass ein schweres OSAS eher zu Organschäden führt verglichen mit einem OSAS in milder oder moderater Ausprägung (Pan et al. 2016).

Das OSAS beeinflusst neben dem kardiovaskulären Sterblichkeitsrisiko auch die karzinombedingte Mortalität. Ursächlich sind vermutlich die intermittierenden Hypoxien, die das Tumorwachstum begünstigen (Nieto et al. 2012).

Ferner können eine ausgeprägte Tagesschläfrigkeit, Konzentrationsstörungen und Depression ursächlich für das erhöhte Mortalitätsrisiko sein, die durch ein unbehandeltes OSAS bedingt sind (Gooneratne et al. 2011). Daraus können häufigere tödliche Verkehrs- und Arbeitsunfälle resultieren. Patienten mit Tagesmüdigkeit und depressiver Symptomatik fällt es zudem schwer, einen gesunden Lebensstil aufrechtzuerhalten, und die Therapie ihrer Komorbiditäten wird oftmals vernachlässigt (Lee et al. 2013; Young et al. 2008). 


\subsection{Fragestellung}

Das OSAS gilt als kardiovaskulärer Risikofaktor. Wie bereits im vorherigen Kapitel beschrieben, scheint es laut einigen größeren Studien einen Einfluss des OSAS auf das kardiovaskuläre Mortalitätsrisiko zu geben. Die bisher verfügbaren Daten sind auf Patienten begrenzt, die von typischen Symptomen des OSAS wie Tagesmüdigkeit betroffen sind. Wie bereits erwähnt bleibt jedoch oftmals das OSAS im klinischen Alltag aufgrund der unspezifischen Symptome unerkannt und ist daher unterdiagnostiziert. In der vorliegenden Studie soll eine Subgruppe (378 Patienten) aus einer großen klinischen Kohortenstudie untersucht werden, die bisher hinsichtlich eines OSAS weitestgehend asymptomatisch waren.

Ziel der vorliegenden Arbeit war es, den Einfluss eines ambulant diagnostizierten obstruktiven Schlafapnoesyndroms bei Patienten mit kardiovaskulären Risikofaktoren auf die Langzeitprognose herauszufinden.

Spezielle Fragestellungen waren hierbei insbesondere:

- Wie hoch ist die Gesamtmortalität bei Patienten mit OSAS? Nimmt die Gesamtmortalität mit zunehmendem Schweregrad zu?

- Gibt es Unterschiede in der geschlechtsspezifischen Mortalität?

- Besteht ein potentieller Einfluss der Variablen Alter und Geschlecht auf die Gesamtmortalität des untersuchten Patientenkollektivs?

- Ist mit zunehmendem Schweregrad des OSAS ein häufigeres Auftreten von $\mathrm{MACE}^{4} \mathrm{zu}$ beobachten?

- Bestehen abseits vom AHI in der Polygraphie messbare Parameter, die mit der Mortalität korrelieren und somit als therapeutische Zielgrößen geeignet sein können?

Ausgehend von den forschungsleitenden Fragen sollen anhand der in der Studie Diast$\mathrm{CHF}^{5}$ im Zeitraum 2004 bis 2015 erhobenen Daten folgende Hypothesen auf ihre Gültigkeit hin überprüft werden:

1. Die Gesamtmortalität nimmt mit zunehmendem Schweregrad des OSAS zu.

2. Das männliche Geschlecht weist eine höhere Mortalitätsrate auf.

\footnotetext{
${ }^{4}$ MACE = Major Adverse Cardiac Event, Erläuterung folgt im Kapitel 3.4

${ }^{5}$ Diast-CHF-Studie $=$ Studie über ,Prävalenz und Verlauf der diastolischen Dysfunktion und der diastolischen Herzinsuffizienz", Erläuterung im Kapitel 2.1
} 
3. Mit zunehmendem Alter nehmen sowohl der AHI als auch das kardiovaskuläre Mortalitätsrisiko zu.

4. Mit zunehmendem Schweregrad des OSAS ist ein häufigeres Auftreten von MACE assoziiert.

5. Es bestehen abseits vom AHI in der Polygraphie messbare Parameter, die auf ein erhöhtes Mortalitätsrisiko schließen lassen. 


\section{Methoden}

\subsection{Studienpopulation}

Die Studienteilnehmer wurden im Rahmen der Studie „Prävalenz und Verlauf der diastolischen Dysfunktion und der diastolischen Herzinsuffizienz“" (kurz Diast-CHF) an der Universitätsmedizin Göttingen rekrutiert. Die Studie wird im Rahmen des Teilprojekts 7 des Kompetenznetzes Herzinsuffizienz (http://knhi.de/studies/tp-7/) durchgeführt. Es handelt sich hierbei um eine prospektive, multizentrische Kohortenstudie. Ein Follow-up wurde bislang jeweils nach 12, 24, 60 und 108 Monaten durchgeführt.

Die Rekrutierung der Patienten wurde in der Zeit von Juli 2004 bis Dezember 2006 organisiert. Partner des Kompetenznetzes sowie niedergelassene Hausärzte und Kardiologen wurden gebeten, Patienten mit Risikofaktoren für die Entwicklung einer diastolischen Herzinsuffizienz für die Studie zu rekrutieren. Insgesamt wurden am Standort Göttingen 1283 Patienten für die Studie rekrutiert. Bei 378 Probanden erfolgte zusätzlich ein Schlafapnoescreening, welches im folgenden Kapitel 2.3 ausführlicher dargestellt wird.

In der folgenden Übersicht sind die geltenden Ein- und Ausschlusskriterien für das Screeningkollektiv zusammengefasst.

Einschlusskriterien:

- Alter zwischen 50 und 85 Jahren

- mindestens einer der folgenden Risikofaktoren für diastolische Dysfunktion:

$\circ$ arterielle Hypertonie

○ Diabetes mellitus

- Zeichen der Arteriosklerose ${ }^{6}$ oder

○ dokumentierte, ärztlich gestellte Diagnose Herzinsuffizienz

- schriftliche Einverständniserklärung zur Studienteilnahme

Ausschlusskriterien:

- bekanntes Schlafapnoesyndrom

- mangelnde Compliance

- unzureichende Kommunikationsfähigkeit aufgrund von schlechten Deutschkenntnissen oder Aphasien

\footnotetext{
${ }^{6}$ Hierzu zählten die symptomatische periphere arterielle Verschlusskrankheit (pAVK), die angiographisch nachgewiesene koronare Herzkrankheit, die Carotisstenose sowie ein stattgehabter Apoplex oder Myokardinfarkt.
} 
- Erkrankungen, die die Einwilligungsfähigkeit einschränken

- geographische Gründe, die eine Studienteilnahme unmöglich machen

Im Rahmen der Follow-up-Untersuchungen konnten die Informationen über neu aufgetretene Hospitalisierungen und MACE direkt bei den Studienteilnehmern erfragt werden. Bei Patienten, die persönlich nicht am Follow-up teilnehmen konnten, wurden die Informationen in einem Telefongespräch eingeholt. Weitere Statusmeldungen und Informationen wie beispielsweise über den Zeitpunkt und Ursache des Todes wurden über Angehörige, Hausärzte, Krankenhausberichte und Standesämter zusammengetragen.

\subsection{Untersuchungen}

\subsubsection{Anamnese}

Von jedem Patienten wurde eine ausführliche Anamnese erhoben. Die Anamnese umfasste sowohl Fragen zur aktuellen Symptomatik auch als auch zur vergangenen Krankheitsgeschichte des Patienten. Zunächst wurden Symptome und Zeichen der Herzinsuffizienz des Patienten erfasst sowie Hospitalisierungen im Krankenhaus oder Rehabilitationseinrichtung jedweder Ursache seit dem letzten Follow-up.

Darauffolgend wurde dokumentiert, ob seit dem letzten Follow-up kardiale Erkrankungen (koronare Herzkrankheit, Myokardinfarkt, Vorhofflimmern oder Kardiomyopathie) neu aufgetretenen sind. Des Weiteren wurde zur Vervollständigung der kardiologischen Anamnese erfragt, ob kardiovaskuläre Interventionen ${ }^{7}$ seit dem letzten Follow-up durchgeführt worden sind.

Ergänzend wurden neu aufgetretene, relevante Nebendiagnosen im Zeitraum seit dem zuletzt durchgeführten Follow-up erfasst (pAVK, zerebrovaskuläre Erkrankung, Synkope, chronisch obstruktive Lungenerkrankung, primäre pulmonale Hypertonie, Depression, HIV-Infektion, chronische Hepatitis B oder C, Leberzirrhose, Malignom, Diabetes mellitus sowie bei Frauen Eintritt der Menopause).

Ferner wurde von jedem Patienten die aktuelle regelmäßige Medikation dokumentiert. Dabei wurde der Medikamentenname mit der jeweiligen Anzahl an Tabletten oder Tropfen pro Tag festgehalten.

\footnotetext{
${ }^{7}$ Kardiovaskuläre Interventionen, worunter koronare oder periphere Revaskularisation, Bypass-OP, sonstige Gefäß-Operationen, Herzklappen-OP, Implantation eines Schrittmachers oder Defibrillators, Z.n. Herztransplantation oder Behandlung mit Assist-Devices, kardiopulmonale Reanimations- oder Defibrillationspflicht zählen.
} 


\subsubsection{Klinische Untersuchungen}

Die klinische Untersuchung beinhaltete die Blutdruckmessung (Blutdruckmessgerät der Marke Omron Modell 705 IT Intellisense), die Ermittlung von Körpergröße und Gewicht (mechanischer Teleskop - Messstab der Marke seca Modell 222 und eine digitale Säulenwaage der Marke seca Modell 704) sowie die Messung des Taillen- und Hüftumfangs mittels eines flexiblen Maßbandes mit Berechnung des Quotienten. Mit Zunahme der ermittelten Waist-to-Hip-Ratio ist aufgrund eines ungünstigen Körperfettverhältnisses ein erhöhtes kardiovaskuläres Risiko vergesellschaftet (de Koning et al. 2007). Anschließend erfolgte eine ausführliche körperliche Untersuchung.

\subsubsection{Basislabordiagnostik}

Bei jedem Patienten wurde eine Blutabnahme in liegender Position durchgeführt. Diese sollte am ruhenden Patienten erfolgen, um einen Anstieg der natriuretischen Peptide und anderer Parameter nach körperlicher Belastung zu vermeiden. Dabei wurden eine SerumMonovette, eine Lithium-Heparinat-Monovette und zwei EDTA-Monovetten abgenommen. Im Labor des Universitätsklinikums Göttingen wurden aus den Blutproben folgende Werte bestimmt: NT-proBNP, Hämoglobinkonzentration, Hämatokrit, Leukozyten- und Thrombozytenzahl, Kreatininkonzentration im Serum, Natrium, Kalium, Gesamtcholesterin, LDL- und HDL-Cholesterin, Harnsäure sowie TSH basal.

\subsubsection{6-Minuten-Gehtest}

Zur Objektivierung der Belastungsfähigkeit des Patienten wurde der 6-Minuten-Gehtest durchgeführt. Der Test hat eine hohe Reliabilität, jedoch ist er eher ungeeignet, um klinische Veränderungen zu detektieren (Rasekaba et al. 2009). Als Wegstrecke diente eine definierte Flurlänge von 100 Metern mit Sitzgelegenheiten in regelmäßigen Abständen. Die Patienten wurden aufgefordert, innerhalb von sechs Minuten die für sie größtmögliche Strecke zurückzulegen, ohne dabei zu rennen oder zu joggen. Die Geschwindigkeit konnte selbst bestimmt werden und gegebenenfalls konnten nötige Pausen eingelegt werden. Der Versuchsleiter sagte alle zwei Minuten die verbliebene Zeit an und ermutigte wiederholt den Patienten verbal. Am Ende des Gehtests wurden die zurückgelegte Distanz, mögliche Unterbrechungen oder ein vorzeitiger Abbruch mit Angabe des Grundes (allgemeine Erschöpfung, Dyspnoe, Angina pectoris, Claudicatio, andere Ursache) dokumentiert. 


\subsubsection{Technische Untersuchungen}

- $\mathrm{EKG}$

Bei jedem Patienten wurde in Ruhe ein 12-Kanal-EKG mit den Ableitungen nach Einthoven, Goldberger und Wilson durchgeführt.

- Echokardiographie

Bei jedem Patienten wurde eine ausführliche transthorakale zweidimensionale echokardiographische Untersuchung durchgeführt. Dazu wurde ein Gerät der Firma Hewlett-Packard Sonos 5500 (Hewlett-Packard, Andover, MA, USA) verwendet. Als Untersucher standen zwei Assistenzärzte der Abteilung Kardiologie und Pneumologie des Uniklinikums Göttingen zur Verfügung.

\subsection{Screening obstruktives Schlafapnoesyndrom}

Aus dem gesamten Risikokollektiv (n=1283) am Standort Göttingen wurde 402 zufällig ausgewählten Patienten die Möglichkeit einer zusätzlichen Untersuchung im Sinne des Schlafapnoescreenings mit zugehöriger Ganzkörperbodyplethysmographie und Polygraphie sowie einem Fragebogen angeboten. Insgesamt nahmen 378 Patienten (94 \%) am Screening teil.

\subsubsection{Ganzkörperbodyplethysmographie}

Die Lungenfunktionsprüfung wurde mit dem Bodyplethysmographen „PowerCube Body“ mit zugehöriger Bodyplethysmographiekabine „Body Scope“ der Firma Ganshorn Medizin Electronic (regulärer Gerätestandort Universitätsklinikum Georg-August-Universität Göttingen, Abteilung Kardiologie und Pneumologie) durchgeführt. Die Messungen wurden von zwei speziell geschulten medizinisch-technischen Assistentinnen ausgeführt, um eine größtmögliche Validität der Daten zu erhalten. Durch die Lungenfunktionsprüfung wurden verschiedene Parameter wie die forcierte Einsekundenkapazität, Vitalkapazität, totale Lungenkapazität und die relative Einsekundenkapazität zur Beurteilung der Lungenfunktion erhoben. Anhand dieser Werte konnten obstruktive, restriktive oder kombinierte Ventilationsstörungen als Ursache für nächtliche Atmungsstörungen ausgeschlossen werden. 


\subsubsection{Polygraphie}

Zur Durchführung der Polygraphie wurde in der Studie das diagnostische Schlafaufzeichnungsgerät „Stardust II“ der Firma Philips Respironics verwendet. Mit Hilfe dieses portablen Gerätes ist es möglich ambulant im häuslichen Rahmen obstruktive Apnoen zu detektieren.

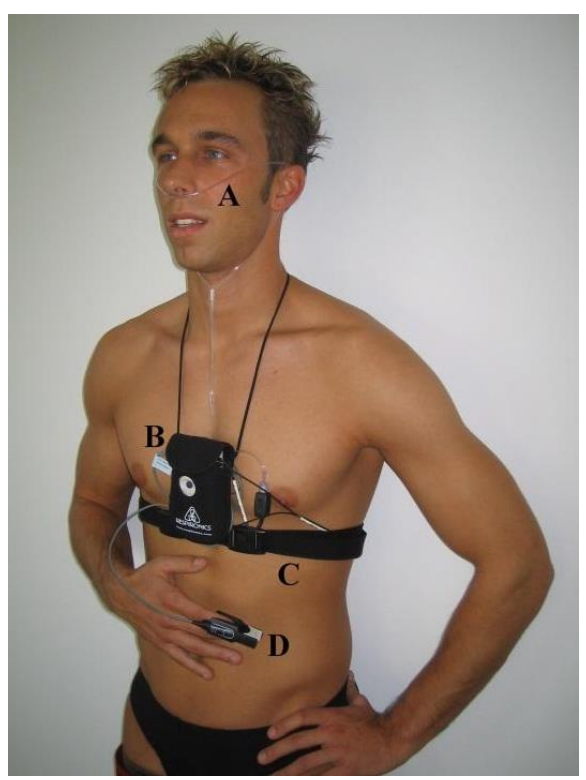

Abbildung 3: Polygraphie-Messgerät am Patienten (mit freundlicher Genehmigung aus Klemmstein 2012)

Das Gerät beinhaltet eine Messeinheit (B) (Größe 11,5 cm x 5,8 cm x 2 cm, Gewicht 102 g), die durch angeschlossene Sensoren kontinuierlich Messwerte aufzeichnet (siehe Abbildung 3). Die Sauerstoffsättigung und Herzfrequenz werden über einen speziellen Fingerclip gemessen (D). Über einen piezoelektrischen Sensor, der sich an einem elastischen Brustgurt befindet, werden Atemexkursionen und Lage des Patienten registriert (C). Der nasale Atemfluss kann mittels einer Nasensonde gemessen und aufgenommen werden (A). Über zwei Sensoren, die sich in den Nasenlöchern befinden, können Schnarchgeräusche aufgezeichnet werden.

Alle gemessenen Daten werden in dem Aufzeichnungsgerät „Stardust II Sleep recorder“ gespeichert. Im Anschluss an den nächtlichen Einsatz werden die gespeicherten Daten über ein angeschlossenes Kabel auf einen handelsüblichen PC überspielt.

Die Auswertung der in der Polygraphie aufgezeichneten Daten erfolgte manuell mit Hilfe eines Auswertungsprogramms der Firma Philips Respironics durch Prof. Dr. med. Lars Lüthje und Dr. med. Daniela Klemmstein.

Für die Analyse wurden Ereignisse von mindestens 10 Sekunden Dauer berücksichtigt.

Ein Ereignis wurde als obstruktive Apnoe gewertet, wenn der über die Nasensonde gemessene Atemfluss unterbrochen wurde bei erhaltenen thorakalen Exkursionen. Davon 
wurde das zentrale Apnoeereignis unterschieden, das durch einen unterbrochenen Atemfluss bei fehlenden Thoraxexkursionen gekennzeichnet ist. Hypopnoen wurden definiert als Reduktion des Atemflusses um $\geq 50 \%$ für mindestens 10 Sekunden mit einer Sauerstoffentsättigung um $\geq 3 \%$ verglichen mit dem vorangegangenen Wert.

Die Patienten wurden gemäß dem Apnoe-Hypopnoe-Index in vier Gruppen eingeteilt:

1. Kein Schlafapnoesyndrom $(\mathrm{AHI}<5 / \mathrm{h})$

2. Mildes Schlafapnoesyndrom (AHI 5-14/h)

3. Moderates Schlafapnoesyndrom (AHI 15-29/h)

4. Schweres Schlafapnoesyndrom (AHI > 30/h)

Zudem beinhaltet die Polygraphie die Messung der Sauerstoffsättigung mittels Pulsoxymetrie. Neben der mittleren Sauerstoffsättigung wurden die kumulative Entsättigungszeit, die minimale Sauerstoffsättigung sowie der Sauerstoffentsättigungsindex berechnet.

\subsubsection{Fragebogen}

Zusätzlich war die Beantwortung eines zweiseitigen Fragebogens Teil des Schlafapnoescreenings. Dieser beinhaltete die subjektive Einschätzung der Tagesschläfrigkeit und Fragen zum Schlafverhalten. Ein besonderes Augenmerk wurde auf die selbstständige Beantwortung der Fragen jedes Studienteilnehmers gelegt.

\section{- Epworth-Schläfrigkeitsskala}

Die ESS ist ein Kurzfragebogen zur Erfassung der Tagesmüdigkeit (entwickelt von dem Australier M. Johns 1991), in dem retrospektiv die Wahrscheinlichkeit für das Einnicken bzw. Einschlafen in acht typischen Alltagssituationen erfragt wird. Hierbei gibt der Patient die subjektive Einschätzung seiner Tagesschläfrigkeit auf einer vierstufigen Skala (von $0=$ „würde niemals einnicken“ bis 3 = „hohe Wahrscheinlichkeit einzunicken“) an. Eine Gesamtpunktzahl von über 10 Punkten ist als pathologisch zu bewerten (Johns 1991).

- Fragebogen zum Schlafverhalten

Ferner wurden die Studienteilnehmer gebeten, einen von Siegrist et al. entworfenen Anamnesebogen zu beantworten. Dieser beinhaltet Fragen zum Schlafverhalten der vergangenen vier Wochen und enthält neben allgemeinen Angaben wie Alter und Geschlecht auch allgemeine und spezielle Fragen zu gestörter Schlafstruktur, nicht erholsamen Schlaf, Tagesmüdigkeit und der Einnahme von Schlafmitteln. Nächtliches 
Schnarchen oder Atemstillstände werden über eine Fremdanamnese angegeben. Die Beantwortung der Fragen erfolgt über eine fünfstufige Skala mit Häufigkeitsangaben von „nie“ (0) bis ,sehr oft“ (5) (Siegrist et al. 1987).

\subsection{Statistische Analyse}

Die erhobenen Daten wurden mit Hilfe der Computersoftware IBM SPSS Statistics Version 24.0 (SPSS Inc., Chicago, IL, USA) statistisch aufbereitet und analysiert. ${ }^{8}$

Vergleiche von Daten wurden in dieser Arbeit mittels Chi-Quadrat-Test oder t-Test (Welch) ermittelt. P-Werte von $<0,05$ wurden als statistisch signifikant definiert.

Für die Überlebenszeitanalysen wurden die Kaplan-Meier-Methode sowie die CoxRegressionsanalyse verwendet.

\section{- Überlebenszeitanalyse}

Mit der Kaplan-Meier-Methode wird die Wahrscheinlichkeit berechnet, dass ein Ereignis bis zu einem bestimmten Zeitpunkt eintritt. Die Zielvariable (in dieser Arbeit der Tod eines Patienten bzw. das Auftreten einer Krankheit) wird nicht zu einem festen Zeitpunkt erhoben, sondern die Ereignisse definieren die Beobachtungsintervalle. Die verschiedenen Zeitintervalle werden dadurch definiert, dass ein Patient verstirbt. Mit Hilfe des KaplanMeier-Schätzers wird die Wahrscheinlichkeit berechnet, dass der Patient das Beobachtungsintervall überlebt. Wenn am Ende des Beobachtungszeitraums das Ereignis nicht eingetreten ist, wird von einer zensierten Beobachtungszeit gesprochen. Die Überlebenszeiten können mit der Kaplan-Meier-Kurve graphisch dargestellt werden. Daraus lassen sich Überlebensraten bestimmen, die angeben, bei wie vielen Patienten bis zu einem bestimmten Zeitpunkt noch kein Ereignis eingetreten ist (Zwiener et al. 2011).

In dieser Arbeit wurden folgende Analysen durch die Kaplan-Meier-Methode erstellt:

○ Überlebensfunktionen für das Screeningkollektiv nach Schlafapnoestadium

○ Überlebensfunktionen für das Screeningkollektiv nach Geschlecht

○ Überlebensfunktionen für die Inzidenz von MACEs für das Screeningkollektiv nach Schlafapnoestadium

○ Überlebensfunktionen von kumulativen Inzidenzen der einzelnen MACE für Studienteilnehmer mit und ohne OSAS

○ Überlebensfunktionen für das Screeningkollektiv nach mittlerer Sauerstoffsättigung

${ }^{8}$ Die Auswertung erfolgte in Zusammenarbeit mit dem Zentrum für Klinische Studien Leipzig Koordinationszentrum für Klinische Studien, Universität Leipzig. 
Zum deskriptiven Vergleich der Überlebenszeiten dienen die Kaplan-Meier-Kurven. Zum statistischen Vergleich der Überlebenszeiten von den Gruppen in einem Beobachtungszeitraum wurde der Log-rank-Test angewendet.

Um den gleichzeitigen Einfluss von mehreren Variablen auf die Überlebenszeit zu untersuchen, wurde eine Cox-Regressionsanalyse verwendet.

Grundlage der Cox-Regressionsanalyse stellt die Hazard-Funktion dar, die das Risiko angibt, genau zum Zeitpunkt t ein Ereignis zu erleiden (entspricht dem Ereignisrisiko, in dieser Arbeit der Tod eines Patienten). Zum Vergleich zweier Gruppen wird der Quotient aus den beiden Hazard-Funktionen gebildet. Daraus errechnet sich die Hazard Ratio (abgekürzt als HR), welches als das Maß, wie groß das Ereignisrisiko in Gruppe 2 im Verhältnis zum Ereignisrisiko in Gruppe 1, zu werten ist.

Voraussetzung für die Cox-Regression ist, dass das Hazard Ratio über die Zeit konstant ist. Dies ist der Fall, wenn das Ereignisrisiko zweier Gruppen proportional zueinander verläuft (entspricht der Annahme von proportionalen Hazards).

Die in der Cox-Regression analysierten Einflussgrößen wurden aufgrund klinischer Zusammenhänge und Literaturrecherchen bestimmt.

- Vergleich von kategorialen und kontinuierlichen Daten

Kontinuierliche Daten wurden als Mittelwert \pm Standardabweichung präsentiert, kategoriale Daten als absolute Zahlen (Prozent). Der Vergleich von kontinuierlichen, normalverteilten Daten erfolgte mittels t-Test (Welch). Für den Vergleich von kategorialen Daten wurde der Chi-Quadrat-Test verwendet. Nicht normalverteilte, kontinuierliche Daten wurden als Median (Interquartile range (IQR)) dargestellt, logarithmiert und mittels t-Test (Welch) verglichen.

Um verschiedene Aspekte des OSAS herauszuarbeiten wurden jeweils Subgruppen aus der am meisten vom Normalwert abweichenden Perzentile der kumulativen Entsättigungszeit, der mittleren und minimalen Sauerstoffsättigung sowie dem Sauerstoffentsättigungsindex gebildet und mit den übrigen $75 \%$ der Patienten verglichen (vgl. Kapitel 3.5). 


\section{Ergebnisse}

\subsection{Charakteristika und Studienpopulation}

Im Rahmen der großen klinischen prospektiven Kohortenstudie (Diast-CHF-Studie) wurden über das Kompetenznetz Herzinsuffizienz 1281 Patienten aus einem hausärztlichen Risikokollektiv am Standort Göttingen rekrutiert. Aus diesem Kollektiv wurde 402 zufällig ausgewählten Teilnehmern zusätzlich die Möglichkeit eines ambulanten Schlafapnoescreenings mit zugehöriger Polygraphie und Ganzkörperbodyplethysmographie angeboten. Insgesamt nahmen 378 Personen am Screening teil; Männer und Frauen waren hier gleich häufig vertreten. Im Folgenden wird diese Gruppe als Screeningkollektiv bezeichnet. Im Vergleich zur Gesamtkohorte der Diast-CHF-Studie waren die teilnehmenden Personen des Screeningkollektivs im Durchschnitt etwas jünger $(66 \pm 7$ Jahre gegenüber $68 \pm 8$ Jahre; p<0,001). Die Verteilung der Geschlechter war in beiden Kollektiven annährend ausgeglichen (weibliches Geschlecht 48 bzw. $50 \%$ ) und hat sich nicht unterschieden.

Das Screeningkollektiv wies sowohl einen niedrigeren Body-Mass-Index als auch signifikant bessere Ergebnisse im 6-Minuten-Gehtest auf. Der Wert des bestimmten NTproBNP fiel in der Gruppe mit Schlafapnoescreening niedriger aus.

Die Teilnehmer des Schlafapnoescreenings wiesen insgesamt weniger Begleiterkrankungen auf. Insbesondere waren sie seltener an Diabetes mellitus erkrankt. Das Vorhandensein von Herzkreislauferkrankungen wie Herzinsuffizienz, arterielle Hypertonie, koronare Herzkrankheit oder Vorhofflimmern war im Screeningkollektiv jeweils signifikant weniger häufig zu beobachten.

In der nachfolgenden Tabelle 2 wird die Kohorte mit Schlafapnoescreening der Kohorte ohne Screening gegenübergestellt. 
Tabelle 2: Klinische Charakteristika der Studienteilnehmer mit und ohne Schlafapnoescreening

\begin{tabular}{|c|c|c|c|}
\hline & $\begin{array}{c}\text { Kein Screening } \\
\quad(\mathbf{n}=903)\end{array}$ & $\begin{array}{l}\text { Screening } \\
(n=378)\end{array}$ & $\mathbf{p}$ \\
\hline \multicolumn{4}{|l|}{ Demographie } \\
\hline Alter (Jahre) & $68 \pm 8$ & $66 \pm 7$ & $<0,001$ \\
\hline Weibliches Geschlecht (\%) & 48 & 50 & 0,388 \\
\hline Body-Mass-Index $\left(\mathrm{kg} / \mathrm{m}^{2}\right)$ & $29,3 \pm 4,9$ & $28,8 \pm 4,5$ & 0,045 \\
\hline \multicolumn{4}{|l|}{ Vitalparameter } \\
\hline Systolischer Blutdruck (mmHg) & $149 \pm 22$ & $152 \pm 20$ & 0,122 \\
\hline Diastolischer Blutdruck (mmHg) & $83 \pm 12$ & $84 \pm 12$ & 0,448 \\
\hline Herzfrequenz (1/min) & $71 \pm 12$ & $71 \pm 12$ & 0,613 \\
\hline $\begin{array}{l}\text { NT-proBNP (pg/ml) } \\
\text { als Median (IQR) }\end{array}$ & $\begin{array}{c}126 \\
(61,9 ; 256)\end{array}$ & $\begin{array}{c}99,3 \\
(51,8 ; 192)\end{array}$ & 0,001 \\
\hline 6-Minuten-Gehtest (m) & $511 \pm 108$ & $554 \pm 84$ & 0,002 \\
\hline \multicolumn{4}{|l|}{ Begleiterkrankungen } \\
\hline Herzinsuffizienz (n, \%) & $128(14 \%)$ & $24(6 \%)$ & $<0,001$ \\
\hline Diabetes mellitus (n, \%) & $248(28 \%)$ & $58(15 \%)$ & $<0,001$ \\
\hline Art. Hypertonie (n, \%) & $825(91 \%)$ & $331(88 \%)$ & 0,037 \\
\hline Hyperlipidämie (n, \%) & $402(45 \%)$ & $149(39 \%)$ & 0,093 \\
\hline Hyperurikämie (n, \%) & $139(15 \%)$ & $68(18 \%)$ & 0,568 \\
\hline Nikotinabusus (n, \%) & $77(9 \%)$ & $34(9 \%)$ & 1,000 \\
\hline Nikotinkarenz (n, \%) & $401(45 \%)$ & $138(37 \%)$ & 0,250 \\
\hline Koronare Herzkrankheit (n, \%) & $221(25 \%)$ & $59(16 \%)$ & $<0,001$ \\
\hline Vorhofflimmern $(\mathrm{n}, \%)$ & $97(11 \%)$ & $4(1 \%)$ & $<0,001$ \\
\hline
\end{tabular}




\subsection{Schlafapnoescreening}

Im Folgenden werden die Ergebnisse des Screeningkollektivs $(n=378)$ detailliert dargestellt. Zunächst wurde die Prävalenz des OSAS bei Patienten mit kardiovaskulären Risikofaktoren ermittelt.

Die in Kapitel 3.2.1 und 3.2.2 vorgestellten Ergebnisse wurden in der Dissertation von Frau Dr. med. Daniela Klemmstein in ähnlicher Darstellung beschrieben (Klemmstein 2012). Sie sind hier der Vollständigkeit halber erwähnt, um ein besseres Verständnis der Basischarakteristika für die Langzeitdaten dieser Patienten zu ermöglichen.

\subsubsection{Prävalenz des obstruktiven Schlafapnoesyndroms}

Bei 238 Patienten $(63 \%)$ des Screeningkollektivs $(n=378)$ wurde ein OSAS (AHI > 5/h) diagnostiziert, wobei in dieser Gruppe das männliche Geschlecht dominierte (57\% der Frauen, 69 \% der Männer). Die 238 OSAS-Patienten wurden je nach Schweregrad des Schlafapnoesyndroms gemäß dem Apnoe-Hypopnoe-Index in drei Gruppen unterteilt. Ein mildes OSAS mit einem AHI zwischen 5 und 14/h zeigte sich bei 149 Teilnehmern (39\%), ein moderates OSAS mit einem AHI zwischen 15 und 29/h wurde bei 59 Patienten (16\%) diagnostiziert und bei 30 Patienten ( $8 \%$ ) lag ein schweres OSAS (AHI $\geq 30 / \mathrm{h}$ ) vor.

Bei 140 Studienteilnehmern (37 \%) fand sich ein AHI $<5 / \mathrm{h}$ und somit konnte ein Schlafapnoesyndrom ausgeschlossen werden (Tabelle 3).

Tabelle 3: Prävalenz für das OSAS des Screeningkollektivs $(n=378)$ in vier Gruppen nach AHI eingeteilt, getrennt nach Geschlecht

\begin{tabular}{|l|c|c|c|c|}
\hline & \multicolumn{3}{|c|}{ Anzahl (n) } & Häufigkeit (\%) \\
\hline AHI (1/h) & gesamt & $\hat{0}$ & + & \\
\hline$<\mathbf{5}$ & $\mathbf{1 4 0}$ & $\mathbf{5 9}$ & $\mathbf{8 1}$ & $\mathbf{3 7}$ \\
\hline$\geq \mathbf{5}$ & $\mathbf{2 3 8}$ & $\mathbf{1 2 9}$ & $\mathbf{1 0 9}$ & $\mathbf{6 3}$ \\
\hline $\mathbf{5 - 1 4}$ & 149 & 69 & 80 & 39 \\
\hline $\mathbf{1 5 - 2 9}$ & 59 & 35 & 24 & 16 \\
\hline$\geq 30$ & 30 & 25 & 5 & 8 \\
\hline gesamt & $\mathbf{3 7 8}$ & $\mathbf{1 8 8}$ & $\mathbf{1 9 0}$ & $\mathbf{1 0 0}$ \\
\hline
\end{tabular}




\subsubsection{Vergleich des Screeningkollektivs nach AHI}

In der folgenden Tabelle 4 wurde das Screeningkollektiv in vier Gruppen (kein OSAS sowie mildes, moderates und schweres OSAS gemäß dem AHI) eingeteilt und jeweils Demographie, Vitalparameter und Begleiterkrankungen verglichen.

Studienteilnehmer mit diagnostiziertem OSAS waren signifikant älter $(p=0,005)$. Im Vergleich der OSAS-Gruppen fiel auf, dass mit steigendem Apnoe-Hypopnoe-Index das Alter der Patienten zunahm. Weiterhin zeigte sich, dass mit zunehmendem Schweregrad die Patienten häufiger männlich waren. Insbesondere waren deutlich mehr Männer an einem schweren OSAS erkrankt (83\%). Im Vergleich des Body-Mass-Index konnte kein signifikanter Unterschied zwischen den Gruppen festgestellt werden. Jedoch zeigte sich bei den Patienten mit obstruktivem Schlafapnoesyndrom eine erhöhte Waist-to-Hip-Ratio $(\mathrm{p}=0,005)$.

Die gemessenen Werte für den Herzinsuffizienzmarker NT-proBNP waren bei den Teilnehmern mit OSAS tendenziell höher als bei denjenigen ohne ein vorliegendes OSAS.

Der Vergleich der zurückgelegten Gehstrecke im 6-Minuten-Gehtest erbrachte keinen signifikanten Unterschied zwischen den Gruppen ( $\mathrm{p}=0,677)$.

Mit zunehmender Schwere des OSAS stieg die Prävalenz der Begleiterkrankungen Herzinsuffizienz, arterielle Hypertonie und Vorhofflimmern, wobei sich hier kein signifikanter Unterschied fand. 
Tabelle 4: Klinische Charakteristika des Screeningkollektivs nach Schweregrad des OSAS

\begin{tabular}{|c|c|c|c|c|c|}
\hline & $\begin{array}{c}\text { AHI }<5 / h \\
(n=140)\end{array}$ & $\begin{array}{c}\text { AHI 5- } \\
14 / h \\
(n=149)\end{array}$ & $\begin{array}{l}\text { AHI 15-29/h } \\
\quad(n=59)\end{array}$ & $\begin{array}{c}\mathbf{A H I} \geq \mathbf{3 0} / \mathbf{h} \\
(\mathbf{n}=\mathbf{3 0})\end{array}$ & $\mathbf{p}$ \\
\hline \multicolumn{6}{|l|}{ Demographie } \\
\hline Alter (Jahre) & $64 \pm 7$ & $67 \pm 6$ & $67 \pm 7$ & $68 \pm 7$ & 0,005 \\
\hline $\begin{array}{l}\text { Weibliches Geschlecht } \\
(\%)\end{array}$ & 58 & 54 & 41 & 17 & $\begin{array}{c}< \\
0,001\end{array}$ \\
\hline BMI $\left(\mathrm{kg} / \mathrm{m}^{2}\right)$ & $28,2 \pm 4,6$ & $28,7 \pm 4,1$ & $29,4 \pm 4,2$ & $28,8 \pm 4,5$ & 0,195 \\
\hline Waist-to-Hip-Ratio & $0,92 \pm 0,08$ & $0,93 \pm 0,08$ & $1,06 \pm 0,77$ & $0,98 \pm 0,08$ & 0,005 \\
\hline \multicolumn{6}{|l|}{ Vitalparameter } \\
\hline $\begin{array}{l}\text { Systolischer Blutdruck } \\
(\mathrm{mmHg})\end{array}$ & $149 \pm 20$ & $154 \pm 21$ & $153 \pm 19$ & $152 \pm 17$ & 0,246 \\
\hline $\begin{array}{l}\text { Diastolischer Blutdruck } \\
(\mathrm{mmHg})\end{array}$ & $83 \pm 12$ & $85 \pm 12$ & $86 \pm 11$ & $85 \pm 10$ & 0,207 \\
\hline Herzfrequenz (1/min) & $71 \pm 12$ & $71 \pm 12$ & $69 \pm 13$ & $67 \pm 12$ & 0,225 \\
\hline $\begin{array}{l}\text { NT-proBNP (pg/ml) } \\
\text { als Median (IQR) }\end{array}$ & $\begin{array}{c}84,3 \\
(44,6 ; 174)\end{array}$ & $\begin{array}{c}94,5 \\
(56,3 ; 190)\end{array}$ & $\begin{array}{c}131 \\
(58,8 ; 292)\end{array}$ & $\begin{array}{c}112 \\
(85,6 ; 194)\end{array}$ & 0,022 \\
\hline $\begin{array}{l}\text { 6-Minuten-Gehtest } \\
\text { (m) }\end{array}$ & $560 \pm 81$ & $552 \pm 85$ & $545 \pm 88$ & $549 \pm 82$ & 0,677 \\
\hline \multicolumn{6}{|l|}{ Begleiterkrankungen } \\
\hline Herzinsuffizienz (n, \%) & $7(5 \%)$ & $8(5 \%)$ & $5(9 \%)$ & $4(13 \%)$ & 0,311 \\
\hline NYHA-Klasse I (\%) & 43 & 25 & 20 & 75 & \\
\hline NYHA-Klasse II (\%) & 57 & 50 & 60 & 0 & \\
\hline NYHA-Klasse III (\%) & 0 & 25 & 20 & 25 & \\
\hline NYHA-Klasse IV (\%) & 0 & 0 & 0 & 0 & \\
\hline Diabetes mellitus $(\mathrm{n}, \%)$ & $20(14 \%)$ & $25(17 \%)$ & $8(14 \%)$ & $5(17 \%)$ & 0,910 \\
\hline Art. Hypertonie (n, \%) & $121(86 \%)$ & $127(85 \%)$ & $55(93 \%)$ & $28(93 \%)$ & 0,313 \\
\hline Hyperlipidämie (n, \%) & $58(41 \%)$ & $52(35 \%)$ & $29(49 \%)$ & $10(33 \%)$ & 0,229 \\
\hline Hyperurikämie (n, \%) & $25(18 \%)$ & $22(15 \%)$ & $10(17 \%)$ & $11(37 \%)$ & 0,042 \\
\hline Nikotinabusus (n, \%) & $15(11 \%)$ & $12(8 \%)$ & $3(5 \%)$ & $4(13 \%)$ & 0,173 \\
\hline Nikotinkarenz (n, \%) & $50(36 \%)$ & $46(31 \%)$ & $28(48 \%)$ & $14(47 \%)$ & \\
\hline $\mathrm{KHK}(\mathrm{n}, \%)$ & $26(19 \%)$ & $19(13 \%)$ & $11(19 \%)$ & $3(10 \%)$ & 0,394 \\
\hline Vorhofflimmern (n, \%) & $2(1 \%)$ & $1(1 \%)$ & 0 & $1(3 \%)$ & 0,473 \\
\hline
\end{tabular}




\subsection{Mortalitätsanalysen des Screeningkollektivs}

Die in den folgenden Kapiteln abgebildeten Kaplan-Meier-Kurven bilden den Beobachtungszeitraum bis 108 Monate (= 9 Jahre) ab. Ereignisse, die nach dieser Zeit aufgetreten sind, wurden nicht mehr berücksichtigt.

Ferner wird darauf hingewiesen, dass in den nachfolgenden Graphiken mit Darstellung des Gesamtüberlebens die Skala der Ordinate auf ein Maß von 50 bis $100 \%$ gekürzt worden ist, um ein anschaulicheres Bild der Kaplan-Meier-Kurven zu erhalten. Zusätzlich sind bei den Kaplan-Meier-Kurven jeweils die Patienten unter Risiko mitaufgeführt.

\subsubsection{Gesamtmortalität}

Ein Ziel der Arbeit war, den Einfluss des ambulant diagnostizierten OSAS bei Patienten mit kardiovaskulären Risikofaktoren auf die 9-Jahres-Mortalität zu untersuchen.

Tabelle 5: Anzahl der verstorbenen Patienten nach AHI und getrennt nach Geschlecht

\begin{tabular}{|l|c|c|c|c|}
\hline & \multicolumn{3}{|c|}{ Tod (n) } & Häufigkeit (\%) \\
\hline AHI (1/h) & gesamt & o & + & \\
\hline$<5$ & $\mathbf{7}$ & $\mathbf{4}$ & $\mathbf{3}$ & $\mathbf{5}$ \\
\hline$\geq \mathbf{5}$ & $\mathbf{2 8}$ & $\mathbf{1 8}$ & $\mathbf{1 0}$ & $\mathbf{1 2}$ \\
\hline $\mathbf{5}-\mathbf{1 4}$ & 20 & 12 & 8 & 13 \\
\hline $\mathbf{1 5}-\mathbf{2 9}$ & 6 & 5 & 1 & 10 \\
\hline$\geq \mathbf{3 0}$ & 2 & 1 & 1 & 7 \\
\hline gesamt & $\mathbf{3 5}$ & $\mathbf{2 2}$ & $\mathbf{1 3}$ & $\mathbf{9}$ \\
\hline
\end{tabular}

Zunächst wurde die Gesamtmortalitätsrate im betroffenen Screeningkollektiv $(n=378)$ in dem Beobachtungszeitraum von 9 Jahren (36483 Patienten-Monate) ermittelt. In der Tabelle 5 ist für das Screeningkollektiv die Anzahl der Todesfälle nach AHI und Geschlecht getrennt dargestellt. Insgesamt sind 35 der 378 Studienteilnehmer (9 \%) verstorben.

Am Schlafapnoescreening nahmen Männer $(n=188)$ und Frauen $(n=190)$ in gleicher Häufigkeit teil. Im Beobachtungszeitraum von 9 Jahren verstarben mehr Männer ( $n=22)$ als Frauen $(\mathrm{n}=13)$.

Wie bereits im vorhergehenden Abschnitt erläutert, wurde bei 238 von den insgesamt 378 
Screeningteilnehmern ein OSAS unterschiedlichen Schweregrades diagnostiziert. Von diesen 238 Patienten sind in dem Beobachtungszeitraum von 9 Jahren insgesamt 28 Personen verstorben (12\%). In der Gruppe mit einem milden Schlafapnoesyndrom ( $\mathrm{n}=149)$ sind 20 Personen verstorben (13\%). Innerhalb der Gruppen mit einem moderaten bzw. schweren OSAS starben $6\left(\begin{array}{lllll}10 & \%\end{array}\right)$ bzw. 2 (7 \%) Studienteilnehmer. Unter den Screeningteilnehmern ohne Nachweis eines OSAS $(n=140)$ sind 7 Personen verstorben $(5 \%)$.

Im Vergleich der Studienteilnehmer ohne OSAS (AHI $<5 / \mathrm{h})$ mit den Teilnehmern, bei denen ein OSAS diagnostiziert wurde (AHI $\geq 5 / \mathrm{h}$ ) (Tabelle 5), zeigte sich ein signifikanter Unterschied des Mortalitätsrisikos zwischen den beiden Gruppen. Für die Patienten mit OSAS fand sich eine erhöhte 9-Jahres-Mortalität (HR 1,90, $95 \%$ - Konfidenzintervall $0,82-4,4, \mathrm{p}=0,023)$.

Um den Einfluss des Alters und des Geschlechts auf die Mortalität zu berücksichtigen, wurde eine Cox-Regressionsanalyse durchgeführt (Tabelle 6). Es zeigte sich weiter ein positiver Zusammenhang zwischen dem Schlafapnoesyndrom und der Mortalität, allerdings war keine statistische Signifikanz mehr vorhanden (HR 1,78, Konfidenzintervall $0,76-4,14, p=0,181)$.

Tabelle 6: Cox-Regressionsanalyse, adjustiert auf Alter und Geschlecht

\begin{tabular}{|l|c|c|c|c|}
\hline & p & Hazard Ratio & 95 \% - Konfidenzintervall für HR \\
\hline OSAS (alle Schweregrade) & 0,181 & 1,78 & 0,76 & 4,14 \\
\hline Alter & $<0,001$ & 1,11 & 1,06 & 1,16 \\
\hline Weibliches Geschlecht & 0,138 & 0,59 & 0,30 & 1,18 \\
\hline
\end{tabular}

Interessant im Zusammenhang mit der Mortalitätsanalyse ist die Frage, ob mit zunehmendem Schweregrad des OSAS ein Anstieg der Mortalitätsrate bei Patienten mit kardiovaskulären Risikofaktoren verbunden ist. Wie in Tabelle 5 dargestellt, zeigten die Ergebnisse eine Abnahme der Mortalität bei zunehmendem AHI von 13,4 \% auf 6,7 \%.

Insgesamt konnte kein signifikanter Unterschied für das Mortalitätsrisiko zwischen den vier einzelnen Patientengruppen gefunden werden ( $\mathrm{p}=0,060 \mathrm{im}$ Log-rank-Test); tendenziell war die Mortalitätsrate bei Patienten mit einem schweren Schlafapnoesyndrom sogar niedriger.

Ferner fiel auf, dass die Gruppe mit einem milden OSAS tendenziell das höchste Mortalitätsrisiko und somit die schlechteste Prognose aufwies, wie in der folgenden Graphik der Kaplan-Meier-Kurven (Abbildung 4) dargestellt wird. 


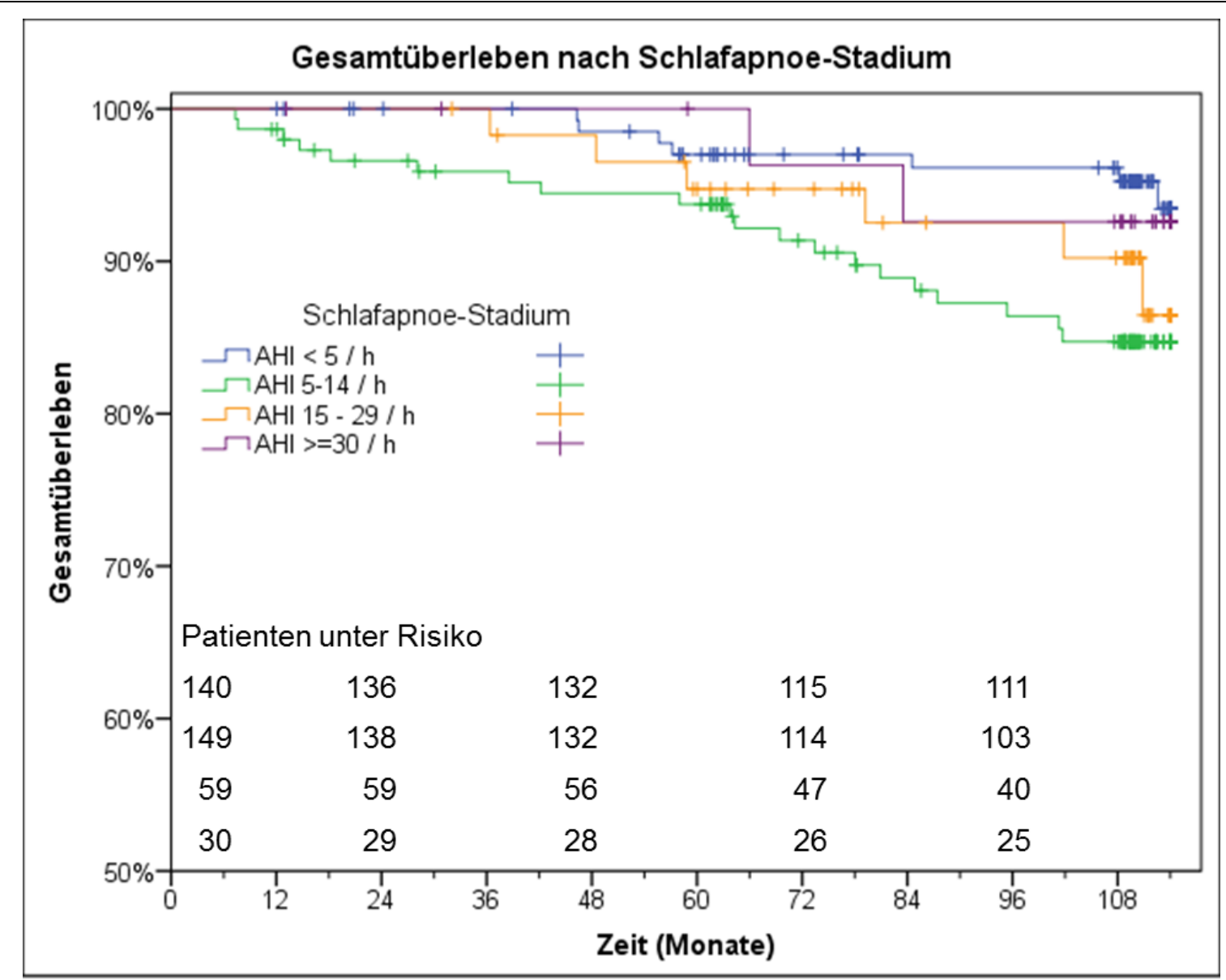

Abbildung 4: Kaplan-Meier-Kurven für das Screeningkollektiv nach Schlafapnoestadium. p=0,060 


\subsubsection{Vergleich und Einfluss des Geschlechts auf die Überlebenszeit}

Wie bereits im vorherigen Abschnitt beschrieben, sind mehr Männer als Frauen innerhalb des Beobachtungszeitraums verstorben (11,7 \% vs. 6,8 \%). Der Vergleich von Männern und Frauen innerhalb des Screeningkollektivs zeigte, dass das weibliche Geschlecht eine tendenziell längere Überlebenszeit aufwies. Dieser Unterschied war jedoch nicht signifikant ( $\mathrm{p}=0,121 \mathrm{im}$ Log-rank-Test). Die folgende Abbildung stellt die beiden KaplanMeier-Kurven der Geschlechter im Vergleich graphisch dar.

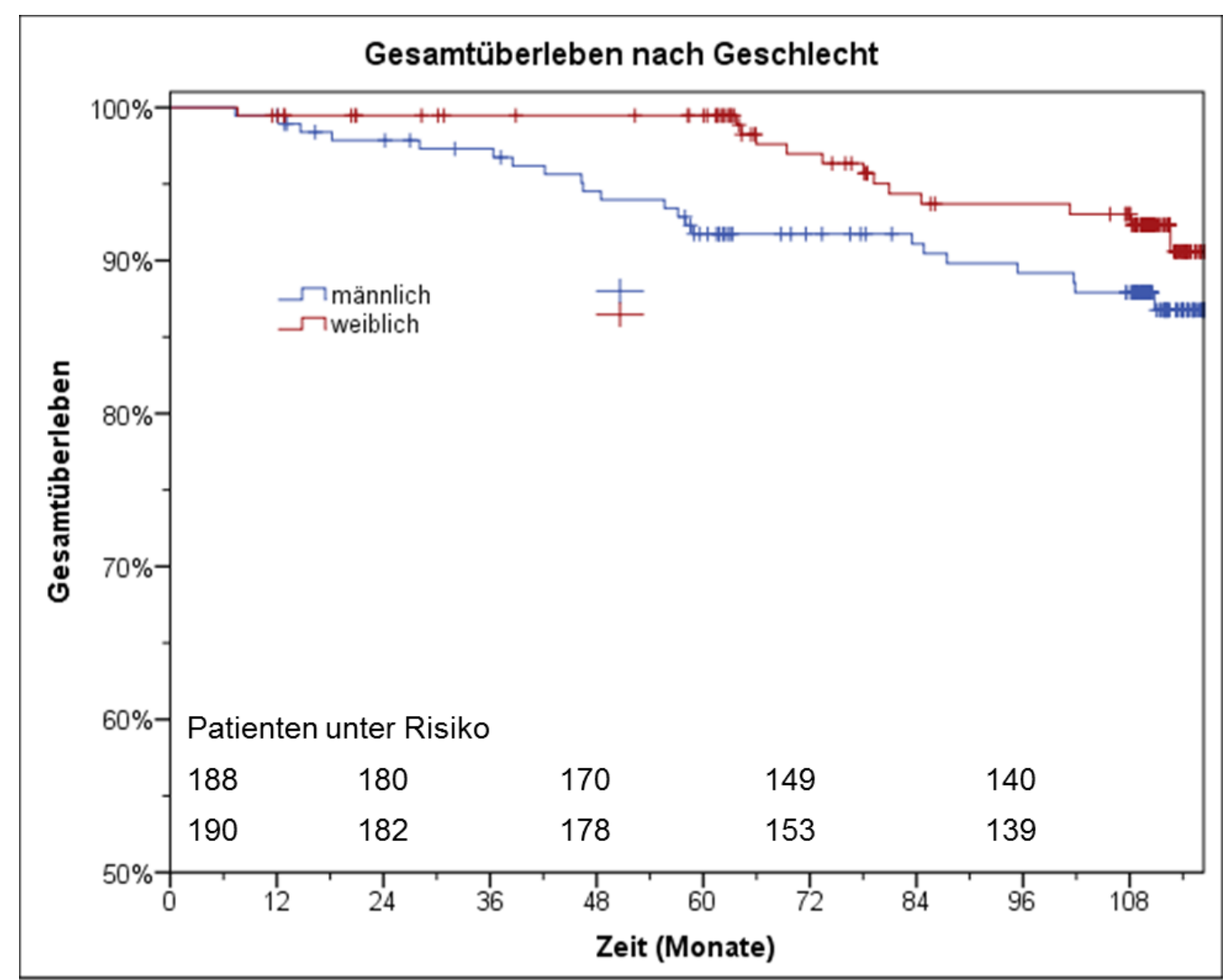

Abbildung 5: Kaplan-Meier-Kurven für das Screeningkollektiv nach Geschlecht. Dargestellt sind alle 378 Teilnehmer am Schlafapnoescreening ( $n=190$ Frauen und n=188 Männer). $p=0,121$

Weiterhin wurde der potentielle Einfluss des Geschlechts auf die Überlebenszeit mittels Cox-Modell untersucht (vgl. Tabelle 7). Das weibliche Geschlecht geht weiterhin mit einem tendenziell geringeren Mortalitätsrisiko einher, jedoch ergab sich keine statistische Signifikanz (HR 0,57, Konfidenzintervall 0,28-1,14, p=0,114).

Ferner war nach Adjustierung auf das Geschlecht das Vorhandensein eines Schlafapnoesyndroms tendenziell mit einem höheren Sterberisiko assoziiert (in allen 3 OSAS-Gruppen HR > 1). Eine statistische Signifikanz für ein erhöhtes Mortalitätsrisiko fand sich allerdings nur für das moderate Schlafapnoesyndrom (HR 2,92, Konfidenzintervall 1,23-6,92, p=0,015). 
Tabelle 7: Ergebnisse der Cox-Regression für die Überlebenszeit, adjustiert auf Geschlecht

\begin{tabular}{|l|c|c|c|c|}
\hline & p & Hazard Ratio & $95 \%$ - Konfidenzintervall für HR \\
\hline OSAS (alle & 0,070 & \multicolumn{3}{|l|}{} \\
Schweregrade) & & & 1,23 & 6,92 \\
$5-14$ & 0,015 & 2,92 & 0,67 & 6,00 \\
$15-29$ & 0,212 & 2,01 & 0,21 & 4,96 \\
$\geq 30$ & 0,989 & 1,01 & 0,28 & 1,14 \\
\hline Weibliches Geschlecht & 0,114 & 0,57 & & \\
\hline
\end{tabular}

\subsubsection{Einfluss der Variablen Geschlecht und Alter auf die Überlebenszeit}

Wie bereits im Kapitel 3.2.2 dargestellt, nahm die Zahl der Apnoen und Hypopnoen mit steigendem Alter zu. Das Alter nahm einen erwartungsgemäß signifikanten Einfluss auf die Schweregradausprägung eines Schlafapnoesyndroms. Zudem gab es einen signifikanten Unterschied zwischen den Geschlechtern, da der Anteil an Männern mit zunehmendem AHI stieg. Wie im vorherigen Kapitel beschrieben, hatten Männer eine kürzere Überlebenszeit als Frauen.

Im Folgenden wurde untersucht, ob ein potentieller, gleichzeitiger Einfluss der Variablen Geschlecht und Alter innerhalb der einzelnen OSAS-Gruppen auf die Überlebenszeit besteht. Die Berechnung erfolgte anhand einer multivariablen Cox-Regressionsanalyse.

Tabelle 8: Ergebnisse der Cox-Regression für die Überlebenszeit, adjustiert auf Alter und Geschlecht

\begin{tabular}{|l|c|c|c|c|}
\hline & p & Hazard Ratio & 95 \% - Konfidenzintervall für HR \\
\hline OSAS (alle Schweregrade) & 0,069 & \multicolumn{3}{|l|}{} \\
\hline AHI (1/h) & & & 1,00 & 5,64 \\
$5-14$ & 0,050 & 2,38 & 0,43 & 4,00 \\
$15-29$ & 0,634 & 1,31 & 0,10 & 2,66 \\
$\geq 30$ & 0,437 & 0,53 & 0,24 & 0,97 \\
\hline Weibliches Geschlecht & 0,040 & 0,48 & 1,07 & 1,18 \\
\hline Alter & $<0,001$ & 1,12 & & \\
\hline
\end{tabular}

Innerhalb der einzelnen OSAS-Gruppen stellte sich insbesondere bei Patienten mit Schlafapnoesyndrom in milder (AHI 5-14/h) und moderater (AHI 15-29/h) Ausprägung ein 2,38- bzw. 1,31-fach erhöhtes Mortalitätsrisiko gegenüber Patienten ohne 
Schlafapnoesyndrom dar. Dieser Effekt war in der Gruppe mit einem milden OSAS grenzwertig signifikant $(\mathrm{p}=0,050)$. Dagegen wiesen Patienten mit einem schweren OSAS ein halb so hohes Mortalitätsrisiko als Patienten ohne OSAS auf (HR 0,53).

Nachdem zusätzlich eine Adjustierung auf das Alter erfolgte, zeigte sich jetzt ein signifikanter Einfluss des Geschlechts auf die Überlebenszeit (HR 0,48, Konfidenzintervall 0,24-0,97, p=0,040). Demnach hatten Frauen gegenüber Männern ein geringeres Risiko zu versterben. Ferner zeigte sich erwartungsgemäß ein signifikanter Einfluss des Alters auf die Überlebenszeit $(\mathrm{p}<0,001)$.

\subsection{Inzidenzen von kardiovaskulären Ereignissen (MACE)}

Nachdem in den vorherigen Kapiteln die Häufigkeit der Schlafapnoe und der Todesfälle innerhalb des Screeningkollektivs dargestellt wurde, soll im folgenden Abschnitt das Auftreten eines MACE (darunter werden in dieser Arbeit die klinischen Ereignisse Tod jedweder Ursache, Hospitalisierung aufgrund einer kardiovaskulären Erkrankung, Myokardinfarkt und Erstdiagnose Herzinsuffizienz zusammengefasst) in dem Beobachtungszeitraum von 9 Jahren untersucht werden. Eine weitere forschungsleitende Frage war, ob mit zunehmendem Schweregrad des OSAS ein häufigeres Auftreten von kardiovaskulären Ereignissen korrelierte. Die folgende Abbildung gibt eine erste Übersicht über die Inzidenz von Todesfällen und MACE in den vier einzelnen OSAS-Gruppen.

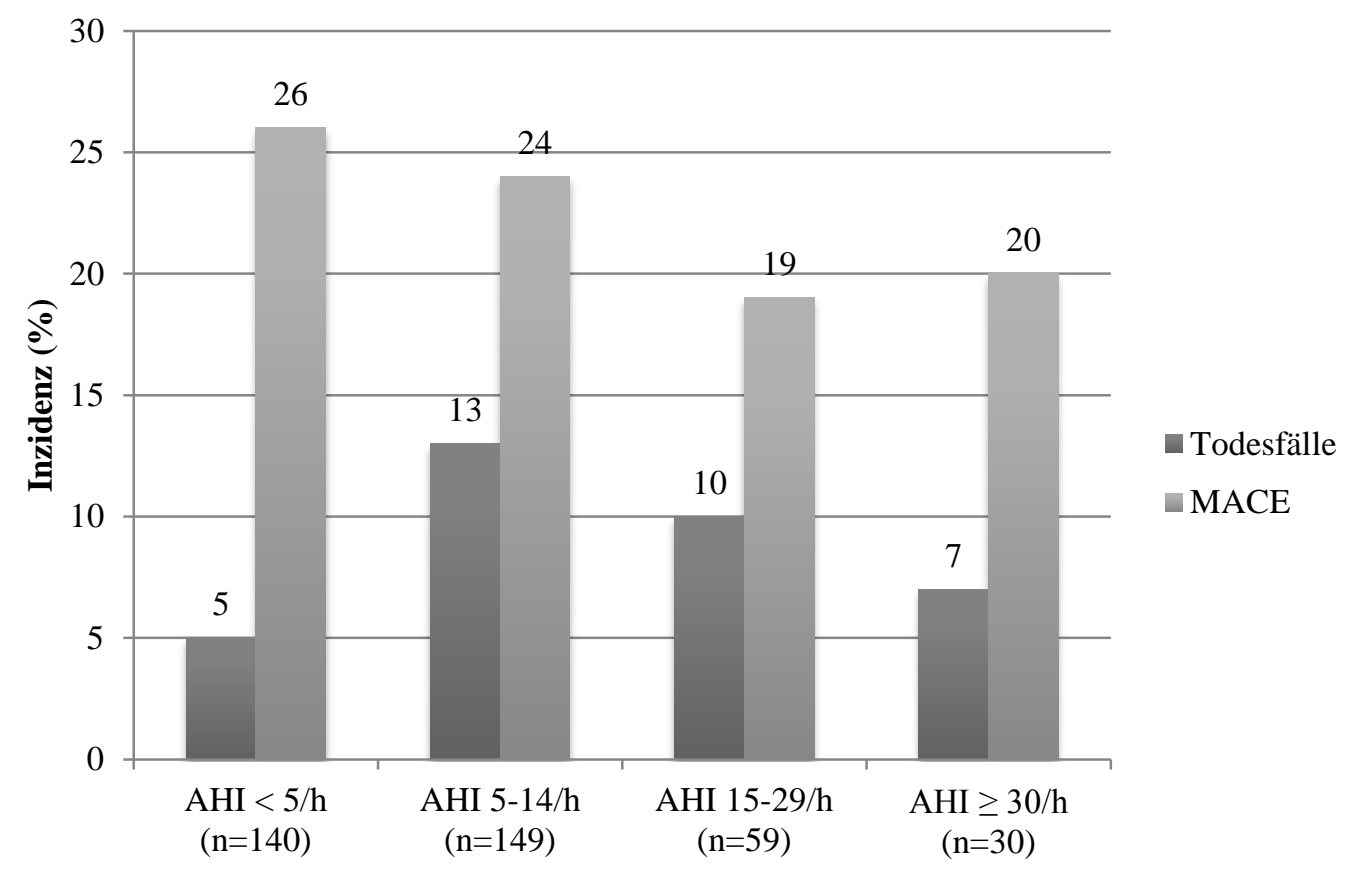

Einteilung des Screeningkollektivs nach AHI

Abbildung 6: Inzidenz von Todesfällen und MACE für das Screeningkollektiv nach AHI als Balkendiagramm dargestellt 
Von den 378 Teilnehmern des Screeningkollektivs erlitten innerhalb des Beobachtungszeitraums von 9 Jahren insgesamt 89 Teilnehmer (24\%) ein MACE. Die relativ häufigsten Ereignisse $(26 \%)$ traten bei Teilnehmern ohne ein OSAS (AHI $<5 / \mathrm{h})$ auf. Während etwa $24 \%$ der Patienten mit einem milden OSAS mit einem MACE symptomatisch wurden, trat ein solches Ereignis bei lediglich jeweils ca. $20 \%$ der Patienten mit einem moderaten sowie schweren OSAS auf (vgl. Tabelle 9 und Abbildung $6)$.

Im Vergleich der Teilnehmer mit und ohne OSAS fiel auf, dass das Vorhandensein eines OSAS keine statistisch signifikante Assoziation für das Auftreten eines MACE aufweist (22\% vs. $26 \%$, HR 0,68, Konfidenzintervall 0,44-1,05, p=0,378).

Tabelle 9: Inzidenzen der einzelnen MACE des Screeningkollektivs sowohl nach AHI getrennt als auch innerhalb des Gesamtkollektivs dargestellt

\begin{tabular}{|c|c|c|c|c|c|}
\hline \multirow[b]{2}{*}{$\begin{array}{l}\text { Erstereignis } \\
\operatorname{MACE}(n, \%)\end{array}$} & \multicolumn{4}{|c|}{ Schlafapnoesyndrom nach AHI (1/h) } & \multirow[b]{2}{*}{$\begin{array}{l}\text { Gesamt } \\
(n=378)\end{array}$} \\
\hline & $\begin{array}{c}<5 \\
(n=140)\end{array}$ & $\begin{array}{c}5-14 \\
(n=149)\end{array}$ & $\begin{array}{l}15-29 \\
(n=59)\end{array}$ & $\begin{array}{c}\geq \mathbf{3 0} \\
(\mathrm{n}=\mathbf{3 0})\end{array}$ & \\
\hline Tod & $7(5 \%)$ & $18(12 \%)$ & $3(5 \%)$ & $2(7 \%)$ & $30(8 \%)$ \\
\hline $\begin{array}{l}\text { Kardiovaskuläre } \\
\text { Hospitalisierung }\end{array}$ & $26(19 \%)$ & $15(10 \%)$ & $5(9 \%)$ & $3(10 \%)$ & $49(13 \%)$ \\
\hline Myokardinfarkt & $2(1 \%)$ & $1(1 \%)$ & $2(3 \%)$ & $1(3 \%)$ & $6(2 \%)$ \\
\hline $\begin{array}{l}\text { Erstdiagnose } \\
\text { Herzinsuffizienz }\end{array}$ & $2(1 \%)$ & $1(1 \%)$ & $1(2 \%)$ & 0 & $4(1 \%)$ \\
\hline \multirow{2}{*}{$\begin{array}{l}\text { Erstereignisse } \\
\text { gesamt }\end{array}$} & \multirow{2}{*}{$\begin{array}{c}37 \\
(26 \%)\end{array}$} & $35(24 \%)$ & $11(19 \%)$ & $6(20 \%)$ & \multirow{2}{*}{$\begin{array}{c}89 \\
(24 \%)\end{array}$} \\
\hline & & & $52(22 \%)$ & & \\
\hline
\end{tabular}


Die in der folgenden Tabelle 10 aufgeführte Cox-Regressionsanalyse, adjustiert auf Alter und Geschlecht, zeigte nun sogar, dass das Vorliegen eines Schlafapnoesyndroms mit einem signifikant niedrigeren Risiko für das Auftreten von MACE korreliert (HR 0,63, 95 \% - Konfidenzintervall 0,41-0,97, p=0,038). Wie zu erwarten, war das Alter mit einem erhöhten Risiko für das Auftreten von kardiovaskulären Ereignissen assoziiert ( $<<0,001)$, während das weibliche Geschlecht das Risiko signifikant reduzierte ( $\mathrm{p}=0,009)$.

Tabelle 10: Cox-Regressionsanalyse für MACE, adjustiert auf Alter und Geschlecht

\begin{tabular}{|l|c|c|c|c|}
\hline & $\mathbf{p}$ & Hazard Ratio & 95 \% - Konfidenzintervall für HR \\
\hline Schlafapnoesyndrom & 0,038 & 0,63 & 0,41 & 0,97 \\
\hline Alter & $<0,001$ & 1,07 & 1,04 & 1,10 \\
\hline Weibliches Geschlecht & 0,009 & 0,57 & 0,37 & 0,87 \\
\hline
\end{tabular}

Wie in Tabelle 9 aufgeführt, wurde als Erstereignis innerhalb des Beobachtungszeitraums von 9 Jahren am häufigsten eine Hospitalisierung aufgrund einer kardiovaskulären Erkrankung beobachtet $(\mathrm{n}=49,13 \%) .6$ Patienten erlitten einen Myokardinfarkt $(2 \%)$ und bei 4 Patienten wurde die Erstdiagnose Herzinsuffizienz (1\%) gestellt. Wie bereits im Kapitel 3.3.1 dargestellt, sind in dem Beobachtungszeitraum insgesamt 35 Patienten des Screeningkollektivs verstorben. Bei 30 Patienten (8\%) trat der Tod ohne vorherige kardiovaskuläre Hospitalisierung, Myokardinfarkt oder Erstdiagnose einer Herzinsuffizienz ein. Bei 289 Teilnehmern (76 \%) trat keines der genannten Ereignisse auf.

Innerhalb der Patientengruppe mit einem ambulant diagnostizierten OSAS (AHI $\geq 5 / \mathrm{h}$ ) war kein Anstieg der kardiovaskulären Ereignisse mit Zunahme des Schweregrads zu verzeichnen. Die folgenden inversen Kaplan-Meier-Kurven (Abbildung 7) veranschaulichen, dass ein höhergradiges Schlafapnoesyndrom nicht mit einem erhöhten Auftreten eines MACE korrelierte.

Zwischen den vier einzelnen Gruppen konnte kein statistischer Unterschied für die Inzidenzen von MACE ermittelt werden ( $\mathrm{p}=0,712 \mathrm{im}$ Log-rank-Test). 


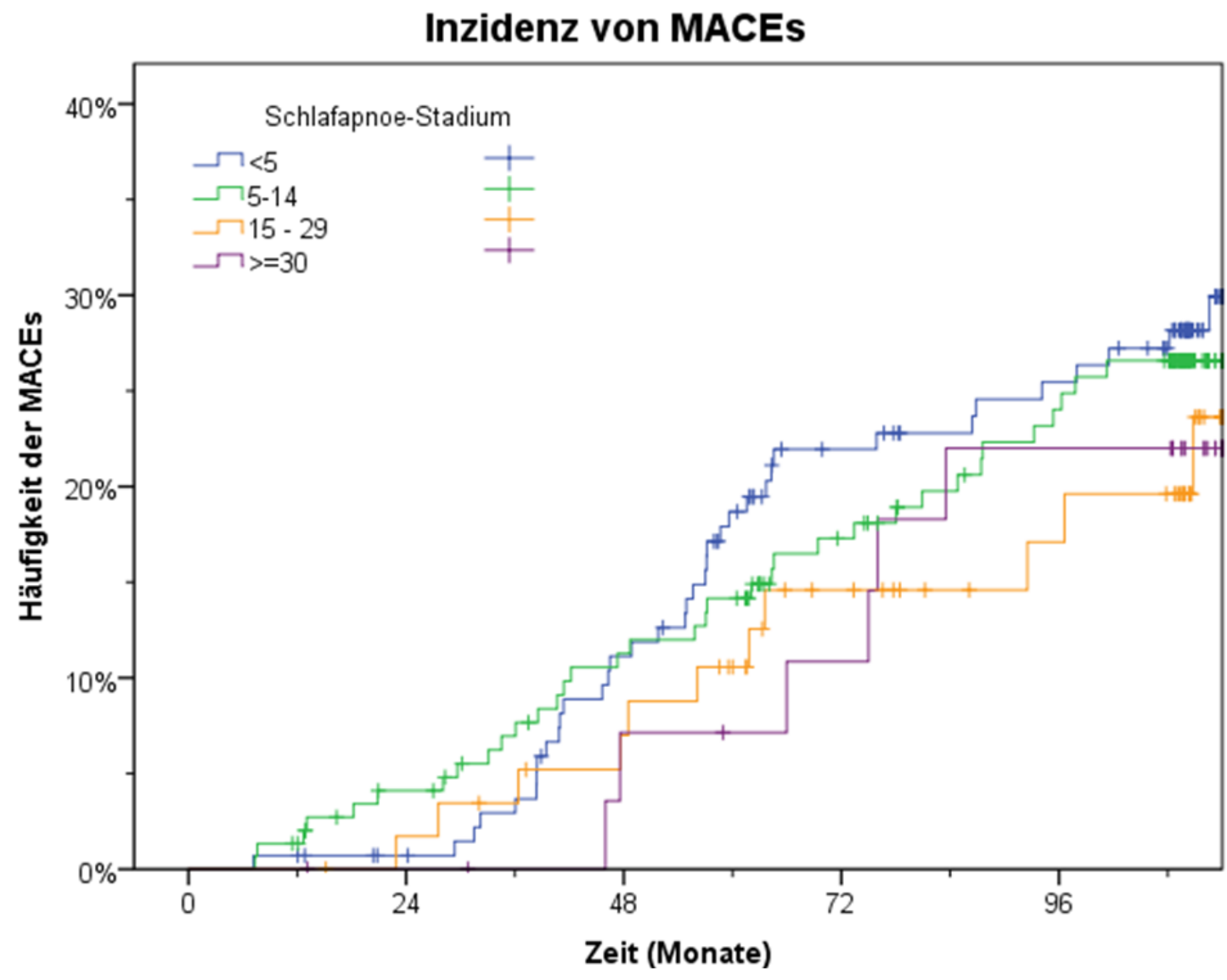

Patienten unter Risiko

$\begin{array}{rrrrr}140 & 135 & 119 & 92 & 84 \\ 149 & 137 & 123 & 103 & 89 \\ 59 & 57 & 52 & 40 & 33 \\ 30 & 29 & 26 & 24 & 21\end{array}$

Abbildung 7: Inverse Kaplan-Meier-Kurven für die Inzidenz von MACEs für das Screeningkollektiv nach Schlafapnoestadium. $\mathrm{p}=0,712$ 
Da die vier betrachteten Ereignisse (Tod, kardiovaskuläre Hospitalisierung, Myokardinfarkt und Erstdiagnose Herzinsuffizienz) als Competing Risks gelten, wurden ergänzend die kumulativen Inzidenzen gerechnet und getestet. Die jeweiligen inversen Kurven der Gruppe mit und ohne OSAS sind in der unten abgebildeten Kaplan-MeierGraphik (Abbildung 8) dargestellt. Ein signifikanter Unterschied zwischen den zwei Gruppen zeigte sich einzig in der Häufigkeit der kardiovaskulären Hospitalisierungen $(\mathrm{p}=0,028)$. Die Studienteilnehmer ohne OSAS waren häufiger hospitalisiert. Dagegen wiesen die Patienten mit OSAS tendenziell eine höhere Mortalität auf. Bezüglich der Häufigkeit von Myokardinfarkten und Erstdiagnose Herzinsuffizienz bestand zwischen den beiden Gruppen kein signifikanter Unterschied.

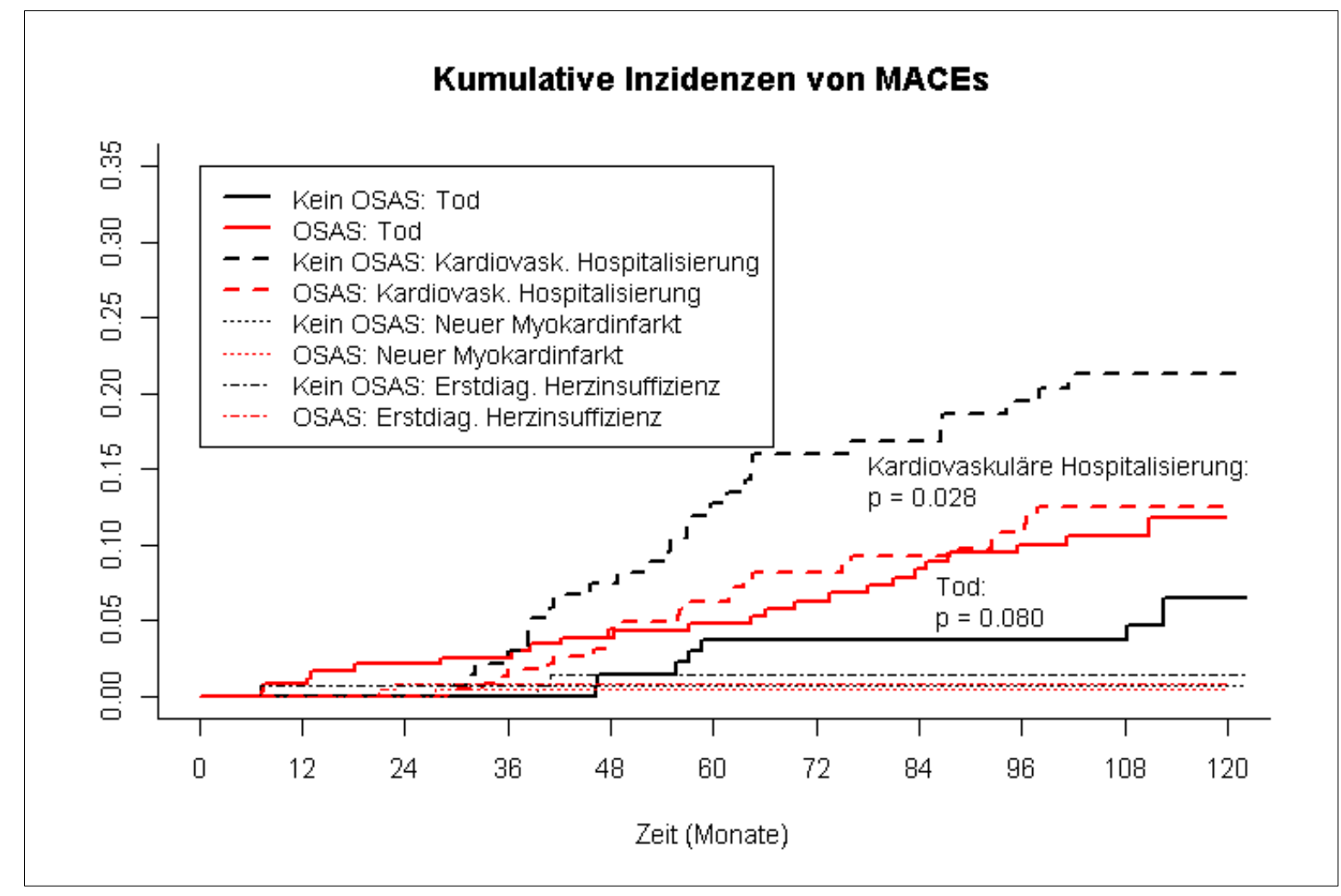

Abbildung 8: Inverse Kaplan-Meier-Kurven von kumulativen Inzidenzen der einzelnen MACE für Studienteilnehmer mit und ohne OSAS. Tod $\mathrm{p}=0,080$, kardiovaskuläre Hospitalisierung $\mathrm{p}=0,028$, Myokardinfarkt $\mathrm{p}=0,712$, Erstdiagnose Herzinsuffizienz $\mathrm{p}=0,595$ 


\subsection{Prognostischer Einfluss von polygraphisch messbaren Parametern}

Im Rahmen der Polygraphie wurden bei den Teilnehmern des Schlafapnoescreenings mittels Pulsoxymetrie die Parameter kumulative Entsättigungszeit (abgekürzt als t90\%) ${ }^{9}$, die mittlere und minimale Sauerstoffsättigung sowie der Sauerstoffentsättigungsindex (abgekürzt als ODI $=$,oxygen desaturation index $\left.{ }^{\text {“ }}\right)^{10}$ erhoben.

Die Ergebnisse des Schlafapnoescreenings inklusive Polygraphie und ESS sowie Bodyplethysmographie der einzelnen AHI-Gruppen wurden ausführlich in der Dissertation von Frau Dr. med. Klemmstein (2012) beschrieben und werden in dieser Arbeit nicht wiederholt dargestellt. Klemmstein beschrieb, dass bei Patienten mit Schlafapnoesyndrom die Sauerstoffentsättigung signifikant ausgeprägter ist. Sowohl die kumulative Entsättigungszeit $190 \%$ als auch der ODI nehmen mit zunehmendem Schweregrad des OSAS zu; kongruent dazu nehmen die mittlere Sauerstoffsättigung sowie die minimale Sauerstoffsättigung ab.

Im folgenden Abschnitt wurde überprüft, ob es neben den klassischen Parametern (z.B. AHI) weitere mittels Polygraphie messbare Parameter gibt, die mit einer erhöhten Gesamtmortalität einhergehen und als therapeutische Zielgröße geeignet sind.

In Tabelle 11 sind die Ergebnisse der $25 \%$ - und $75 \%$ - Perzentilen sowie des Medians der genannten vier relevanten Parameter der Polygraphie aufgeführt. Aufgrund von technisch bedingten Messfehlern in der Polygraphie unterscheidet sich die Anzahl der in die Analyse eingehenden Messergebnisse zwischen den einzelnen Parametern.

\footnotetext{
${ }^{9}$ Die kumulative Entsättigungszeit entspricht der Dauer von Sauerstoffsättigungen $<90 \%$ während der nächtlichen Schlafzeit.

${ }^{10}$ Der Sauerstoffentsättigungsindex ist definiert als die Anzahl von Sauerstoffentsättigungen um mindestens $3 \%$ pro Stunde Schlaf (Hoshino et al. 2016).
} 
Tabelle 11: Anzahl der Patienten mit validem bzw. fehlerhaftem Messergebnis sowie $25 \%$ - und $75 \%$ Perzentile und Median der gemessenen Parameter

\begin{tabular}{|c|c|c|c|c|}
\hline & $\begin{array}{c}25 \%- \\
\text { Perzentil }\end{array}$ & Median & $\begin{array}{c}75 \%- \\
\text { Perzentil }\end{array}$ & $\begin{array}{l}\text { Anzahl der Patienten mit } \\
\text { validem / fehlerhaftem } \\
\text { Messergebnis (n) }\end{array}$ \\
\hline Mittlere $\mathrm{O}_{2}$-Sättigung (\%) & 93 & 94 & 95 & $342 / 36$ \\
\hline $\begin{array}{l}\text { Kumulative } \\
\text { Entsättigungszeit t90\% } \\
\text { (min) }\end{array}$ & 1 & 5 & 30 & $352 / 26$ \\
\hline ODI (1/h) & 4,8 & 10,7 & 20,25 & $352 / 26$ \\
\hline $\begin{array}{l}\text { Minimale } \mathrm{O}_{2} \text { - Sättigung } \\
(\%)\end{array}$ & 75 & 81 & 85 & $332 / 46$ \\
\hline
\end{tabular}

\subsubsection{Mittlere Sauerstoffsättigung}

Tabelle 12: Vergleich der mittleren Sauerstoffsättigung der Teilnehmer nach Perzentile; je Anzahl und Todesfälle. $\mathrm{p}=0,016$

\begin{tabular}{|l|c|c|c|c|}
\hline $\begin{array}{l}\text { Mittlere } \\
\text { O2-Sättigung } \\
(\%)\end{array}$ & $\begin{array}{c}\text { Anzahl } \\
(\mathbf{\%})\end{array}$ & $\begin{array}{c}\text { Verstorben } \\
(\mathbf{n})\end{array}$ & $\begin{array}{c}\text { Häufigkeit } \\
(\%)\end{array}$ & $\begin{array}{c}\text { Zensiert } \\
\text { (n) }\end{array}$ \\
\hline$<93$ & 64 & 11 & 17 & 53 \\
\hline$\geq 93$ & 278 & 22 & 8 & 256 \\
\hline gesamt & 342 & 33 & 10 & 309 \\
\hline
\end{tabular}

Im Rahmen der Polygraphie wurde mittels Pulsoxymetrie die mittlere Sauerstoffsättigung ermittelt. Eine mittlere Sauerstoffsättigung von < $93 \%$ wiesen 64 Patienten auf, von denen 11 Personen verstorben sind (17\%). Dementsprechend wurde bei 278 Studienteilnehmern eine mittlere Sauerstoffsättigung von $\geq 93 \%$ gemessen. Von diesen Teilnehmern sind im Beobachtungszeitraum von 9 Jahren 22 Personen verstorben $(8 \%)$.

Im Vergleich zeigte sich für Studienteilnehmer mit einer mittleren Sauerstoffsättigung von $<93 \%$ ein signifikant erhöhtes Mortalitätsrisiko $(\mathrm{p}=0,016)$. Die folgende Graphik (Abbildung 9) stellt die entsprechenden Kaplan-Meier-Kurven dar. Hinzugefügt werden muss allerdings, dass eine Aufspaltung der Kurven wie dargestellt erst nach 60 Monaten zu beobachten ist. Damit ist die Annahme von proportionalen Hazards nicht gegeben. Daher wurde zusätzlich eine Cox-Regression mit zeitabhängigen Kovariaten berechnet, die keinen statistischen Unterschied zwischen den beiden Gruppen aufzeigte $(p=0,166)$. 


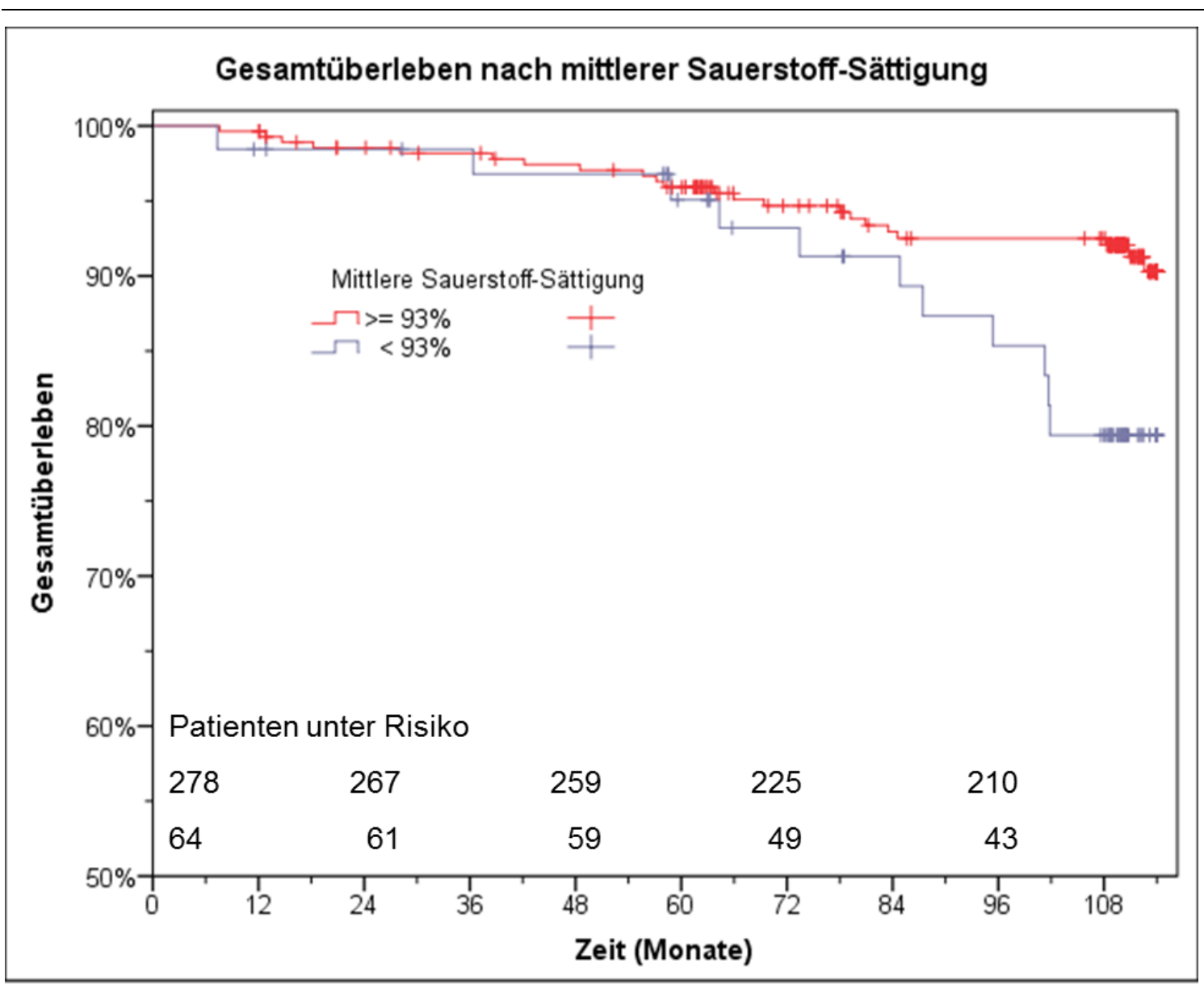

Abbildung 9: Kaplan-Meier-Kurven für das Screeningkollektiv nach mittlerer Sauerstoffsättigung. p=0,016 


\subsubsection{Kumulative Entsättigungszeit, Sauerstoffentsättigungsindex und minimale} Sauerstoffsättigung

Tabelle 13: Vergleich der Ergebnisse der t90\%, des ODI und der minimalen Sauerstoffsättigung der Teilnehmer nach Perzentile; je Anzahl und Todesfälle

\begin{tabular}{|c|c|c|c|c|}
\hline & $\begin{array}{c}\text { Anzahl } \\
\text { (n) }\end{array}$ & $\begin{array}{c}\text { Verstorben } \\
\text { (n) }\end{array}$ & $\begin{array}{c}\text { Häufigkeit } \\
(\%)\end{array}$ & $\begin{array}{c}\text { Zensiert } \\
\text { (n) }\end{array}$ \\
\hline \multicolumn{5}{|c|}{ Kumulative Entsättigungszeit t90\% (min) } \\
\hline$\leq \mathbf{3 0}$ & 264 & 21 & 8 & 243 \\
\hline$>30$ & 88 & 13 & 15 & 75 \\
\hline \multicolumn{5}{|c|}{ ODI (1/h) } \\
\hline$\leq 20,25$ & 264 & 24 & 9 & 240 \\
\hline$>20,25$ & 88 & 10 & 11 & 78 \\
\hline \multicolumn{5}{|c|}{ Minimale Sauerstoffsättigung (\%) } \\
\hline$\geq 75$ & 255 & 23 & 9 & 232 \\
\hline$<75$ & 77 & 8 & 10 & 69 \\
\hline
\end{tabular}

Entsprechende Berechnungen wurden für die übrigen drei Parameter kumulative Entsättigungszeit, ODI und minimale Sauerstoffsättigung durchgeführt.

13 von 88 Patienten (15\%) mit t90\% > 30 Minuten verstarben im Vergleich zu 21 von 264 (8 \%) Patienten mit t90\% von $\leq 30$ Minuten. Für Patienten mit einer kumulativen Entsättigungszeit t90\% von > 30 Minuten wurde ein tendenziell erhöhtes Mortalitätsrisiko gefunden $(\mathrm{p}=0,057)$.

Hingegen wurden im Vergleich für Patienten mit einem ODI von über oder $\leq 20,25 / \mathrm{h}$ $(\mathrm{p}=0,578)$ sowie für Patienten mit einer minimalen Sauerstoffsättigung von unter oder $\geq$ $75 \%(\mathrm{p}=0,830)$ keine Unterschiede in der Überlebenszeit nachgewiesen. 


\section{Diskussion}

In der vorliegenden Arbeit wurde zunächst das OSAS definiert und ein Überblick über die klinischen und demographischen Parameter und Überlebensdaten der Patienten gegeben, die am Standort der Universitätsmedizin in Göttingen innerhalb der letzten 9 Jahre im Rahmen einer großen prospektiven Kohortenstudie diagnostiziert und beobachtet wurden. Neben den Daten der Gesamtkohorte wurden in der Arbeit insbesondere die Daten von 378 Patienten mit Schlafapnoescreening analysiert. Das Schlafapnoescreening erfolgte ambulant mittels Polygraphie bei Patienten mit kardiovaskulären Risikofaktoren.

In der Analyse wurden sowohl die klinischen Charakteristika als auch Überlebensraten der gescreenten Studienpopulation sowie verschiedener Subgruppen ermittelt und mit Hilfe von Log-rank-Tests und Cox-Regressionsanalysen verglichen. Ein weiteres Ziel war es, einen prognostischen Prädiktor für die Mortalität durch einen in der Polygraphie messbaren und reproduzierbaren Parameter zu ermitteln.

In diesem Kapitel sollen die im vorherigen Abschnitt dargestellten Ergebnisse im Hinblick auf die in Kapitel 1.3 vorgestellten forschungsleitenden Fragen und Forschungshypothesen diskutiert werden. Ferner sollen die Ergebnisse in Bezug zu den Ergebnissen anderer Studien gesetzt und in den bisherigen Wissensstand der Forschung zu diesem Thema eingeordnet werden.

\subsection{Prävalenz des OSAS im Vergleich mit anderen Studien}

In der vorliegenden Studienpopulation wurde bei insgesamt $63 \%(n=238)$ der Teilnehmer ein OSAS mit einem AHI $\geq 5 / h$ festgestellt. Bei $39 \%(n=149)$ der Studienteilnehmer fand sich ein mildes OSAS mit einem AHI zwischen 5/h und 14/h. $24 \%(n=89)$ aller gescreenten Patienten (16\% Männer und $8 \%$ Frauen) wiesen ein OSAS in moderater oder schwerer Ausprägung (entsprechend einem AHI $\geq 15 / \mathrm{h})^{11}$ auf.

In anderen vergleichbaren Studien fanden sich Prävalenzen zwischen 1 und $17 \%$ variierend für das moderate OSAS sowie von 3 bis $28 \%$ für das milde OSAS (Bixler et al. 1998; Punjabi et al. 2009; Young et al. 2002a).

In der Wisconsin Sleep Cohort Study von 1993 mit einem im Vergleich zur vorliegenden Arbeit etwas größeren Studienkollektiv ( $\mathrm{n}=625)$ wurde bei $24 \%$ der Männer und $9 \%$ der Frauen im Alter von 30 bis 60 Jahren ein $A H I \geq 5 / h$ bzw. bei $9 \%$ der Männer und $4 \%$ der

\footnotetext{
${ }^{11}$ Die Einteilung AHI $\geq 15 / \mathrm{h}$ wurde gewählt, um die Vergleichbarkeit mit anderen Studien zu erleichtern.
} 
Frauen ein AHI $\geq 15 / \mathrm{h}$ festgestellt (Young et al. 1993). In der weiteren Beobachtungsphase ( $\mathrm{n}=1522)$ stieg die Prävalenz wesentlich an; bis zum Jahr 2011 wiesen $13 \%$ der Männer und $6 \%$ Frauen (30-70 Jahre) einen AHI $\geq 15 / \mathrm{h}$ auf (Malhotra et al. 2015; Peppard et al. 2013). Im Vergleich zu unserem Kollektiv waren die Patienten deutlich jünger (48 vs. 66 Jahre) und wiesen deutlich weniger Begleiterkrankungen bei vergleichbarem BMI und Geschlechterverteilung auf (Young et al. 2008).

Vergleichbar mit dem vorliegenden Ergebnis wurde in einem von Duran untersuchten spanischen Kollektiv im Alter von 30 bis 70 Jahren ein $A H I \geq 15 / h$ bei $14 \%$ der Männer und $7 \%$ der Frauen gefunden (Duran et al. 2001).

Punjabi et al. (2009) ermittelten in der prospektiven SHHS eine ähnliche Prävalenz für ein moderates und schweres OSAS von $17 \%$. Das Studienkollektiv war wesentlich größer, jedoch waren die Charakteristika wie Geschlecht, BMI, Nikotinabusus und Anzahl an Vorerkrankungen wie arterielle Hypertonie und KHK annähernd vergleichbar mit unserem Screeningkollektiv. Ebenso war die mittlere Beobachtungszeit mit 8,2 Jahren von ähnlicher Dauer.

Im Vergleich ist die Prävalenz eines OSAS mit einem AHI $\geq 5 / \mathrm{h}$ in der vorliegenden Kohorte deutlich höher gegenüber den genannten großen Kohortenstudien. Die Häufigkeit eines moderaten oder schweren OSAS liegt ebenso tendenziell höher.

Dabei ist die zugrunde gelegte Definition des Schlafapnoesyndroms im Vergleich der Studien zu berücksichtigen. Unterschiede bestehen darin, ob für die Diagnosestellung eines OSAS neben dem AHI auch eine typische Klinik, wie beispielsweise von der AASM bei einem AHI $<15 / \mathrm{h}$ definiert, gefordert ist (Malhotra et al. 2015). In der vorliegenden Arbeit wurde die Einteilung nur anhand des AHI ohne Berücksichtigung der klinischen Symptomatik vorgenommen. Um die Symptomatik der Patienten zu erfragen, kann als ein Screeninginstrument der ESS zur Erfassung der Tagesschläfrigkeit herangezogen werden. In der vorliegenden Kohorte lag die mittlere erreichte Punktzahl bei 5,5 für Patienten mit mildem OSAS bzw. 7 für Patienten mit moderatem bis schwerem OSAS ${ }^{12}$. Dieses Ergebnis deutet darauf hin, dass im untersuchten Screeningkollektiv keine höhergradige Symptomatik vorliegt und die Patienten in Hinblick auf ein OSAS überwiegend als asymptomatisch anzusehen sind.

Jedoch sind in aktuelleren Studien auch deutliche Abweichungen zu den zuvor genannten Prävalenzangaben zu finden. In einer Studie von Lee et al. (2013) mit 2240 Probanden litten 55,8 \% der Teilnehmer an einem moderaten oder schweren OSAS. Dieses

\footnotetext{
${ }^{12}$ Die Ergebnisse des Schlafapnoescreenings inklusive ESS der einzelnen AHI-Gruppen wurden ausführlich in der Dissertation von Frau Dr. Klemmstein (2012) beschrieben.
} 
Teilnehmerkollektiv war etwas jünger und der BMI niedriger, jedoch war der Anteil an Männern mit 74,5 \% wesentlich größer als in anderen Studien.

In einer in Finnland durchgeführten Studie lag der Anteil der Patienten, bei denen mittels ambulanter Polygraphie ein moderates oder schweres OSAS diagnostiziert wurde, mit 49,6 \% ebenfalls deutlich höher (Muraja-Murro et al. 2013).

Die deutlichen Unterschiede in der Prävalenz des OSAS zu unserer Arbeit (55,8\% bzw. 49,6 \% vs. $24 \%$ ) lassen sich durch die untersuchten Studienpopulationen erklären. Im Gegensatz zu unserem Kollektiv, das als Teil einer großen Studie bisher weitestgehend asymptomatisch bezüglich einer SBAS war, wurden sowohl bei Lee als auch bei MurajaMurro Patienten in die Studie eingeschlossen, bei denen das Symptom Schnarchen vorhanden war oder ein OSAS bereits diagnostiziert bzw. aufgrund einer typischen Anamnese ein OSAS vermutet wurde. Die deutlich höheren Prävalenzen können durch das vorselektierte Studienkollektiv bedingt sein.

Weiterhin wurden unterschiedliche Definitionen für Hypopnoen festgelegt, so dass der Wert des AHI je nach Schlaflabor und Auswertungsmethode variieren kann (Jordan et al. 2014). Für die Einteilung des OSAS nach dem AHI hat sich inzwischen die in dieser Arbeit vorgenommene Einteilung in drei Schweregrade durchgesetzt.

Neben den Definitionen hat auch die gewählte Untersuchungsmethode Einfluss auf die Prävalenz. In den verschiedenen Studien variiert die Art des Schlafapnoescreenings. In den Studien von Young, Bixler, Lee und Duran wurde eine stationäre Polysomnographie durchgeführt. Hingegen erfolgte die Untersuchung der Studienteilnehmer in der SHHS, in den Studien von Muraja-Murro und Marshall sowie in der vorliegenden Arbeit im ambulanten Rahmen mittels portabler Polygraphie (Pan et al. 2016; Quan et al. 1997). Eine Übersichtsarbeit und Metaanalyse zur Diagnose der Schlafapnoe fand eine reduzierte Zuverlässigkeit der Polygraphie gegenüber der Polysomnographie mit häufigeren falschpositiven Ergebnissen (Ross et al. 2000). Dennoch könnte zukünftig die Polygraphie als ambulante Untersuchungsmethode die Polysomnographie im Schlaflabor als Goldstandard ablösen. Dieser Wandel beruht vor allem auf den niedrigeren Kosten sowie der einfacheren Verfügbarkeit der Polygraphie. Inwieweit die Polygraphie auch eine gleichwertige Methode zur Erkennung des OSAS darstellt, bleibt gegenwärtig noch offen (Malhotra et al. 2015).

Der natürliche Verlauf des OSAS impliziert nächtliche Schwankungen der Ausprägung und Anzahl von Apnoen und Hypopnoen (Freedman 2015). Diese Schwankungen bedingen, dass einmalige schlafmedizinische Untersuchungen den Schweregrad eines OSAS 
möglicherweise nicht korrekt erfassen (McNicholas 2008). Eine methodische Limitation dieser Arbeit ist, dass pro Patient nur eine nächtliche Messung zur Diagnosestellung eines OSAS durchgeführt worden ist.

\subsection{Gesamtmortalität des Screeningkollektivs im Vergleich mit anderen Studien}

Ein großer Teil dieser Arbeit fokussiert sich auf die Erhebung der Gesamtmortalität bei Patienten mit kardiovaskulären Risikofaktoren und OSAS in dem Beobachtungszeitraum von 9 Jahren. In der gesamten untersuchten Kohorte beträgt die Mortalitätsrate $9 \%$. Betrachtet man nur die Studienteilnehmer, bei denen ein OSAS diagnostiziert wurde, liegt die Sterblichkeitsrate bei $12 \%$.

In der Literatur sind Angaben für die Mortalitätsrate für Patienten mit einem OSAS zwischen 3,4 \% (Lee et al. 2013) und 19,9 \% zu finden (Punjabi et al. 2009). Die Gesamtmortalität der vorliegenden Arbeit mit $12 \%$ liegt in diesem Bereich. Ein ähnliches Ergebnis zeigte die Studie von Young et al. (2008) mit einer Gesamtmortalität von 9,3\%, wobei hier der Beobachtungszeitraum mit durchschnittlich 13,8 Jahren länger gewesen ist. Bei Lee et al. (2013) betrug der Beobachtungszeitraum nur 5,1 Jahre im Mittel, was mitunter die niedrigere Mortalitätsrate erklären könnte.

Im Vergleich der vorliegenden Daten ergibt sich ein signifikanter Unterschied bezüglich des Mortalitätsrisikos zwischen den Studienteilnehmern ohne und mit diagnostiziertem OSAS (5\% vs. $12 \%$ ). Das Vorhandensein eines OSAS (AHI $\geq 5 / \mathrm{h}$ ) geht mit einem signifikant erhöhten Mortalitätsrisiko einher (HR 1,90, Konfidenzintervall 0,82-4,4, $\mathrm{p}=0,023)$.

Ähnliche Ergebnisse zeigten sich in der Wisconsin Sleep Cohort Study, in der auch ein erhöhtes, jedoch statistisch nicht signifikantes Mortalitätsrisiko für Patienten mit einem milden und moderaten OSAS gegenüber OSAS-freien Patienten ermittelt wurde. Im Gegensatz zu der vorliegenden Arbeit wurde jedoch gezeigt, dass ein schweres OSAS mit einem fast dreifach erhöhten Mortalitätsrisiko signifikant $(\mathrm{p}=0,008)$ assoziiert ist (Young et al. 2008). Die Mehrzahl der bisher publizierten Studien beschreiben eine Assoziation zwischen einer schlafbezogenen Atmungsstörung und erhöhten Gesamtmortalität (Morimoto et al. 2012; Punjabi et al. 2009; Young et al. 2008).

Innerhalb der vorliegenden Studienkohorte mit diagnostiziertem OSAS (AHI $\geq 5 / \mathrm{h}$ ) war entgegen der Forschungshypothese überraschenderweise $\mathrm{zu}$ beobachten, dass die Mortalitätsrate mit zunehmendem Schweregrad des OSAS nicht ansteigt und tendenziell bei Patienten mit einem schweren Schlafapnoesyndrom sogar niedriger ist. 
Interessanterweise zeigt sich das höchste Mortalitätsrisiko bei Patienten mit einem milden OSAS.

Im Gegensatz zu unserer Arbeit zeigte sich in anderen Studien, dass mit zunehmendem Schweregrad des OSAS die Mortalität anstieg. Sowohl bei Young und Mitarbeitern als auch in den Studien von Punjabi, Lee und Muraja-Murro wurde das höchste Mortalitätsrisiko bei Patienten mit einem schweren OSAS gefunden (Lee et al. 2013; Muraja-Murro et al. 2013; Punjabi et al. 2009; Young et al. 2008). Ähnliche Ergebnisse lieferte die Wisconsin Sleep Cohort Study. Patienten mit einem schweren OSAS (AHI $\geq$ 30/h) wiesen im Vergleich zu Patienten mit einem AHI > 5/h ein 2,7-fach erhöhtes Mortalitätsrisiko auf (Young et al. 2008). Noch deutlicher zeigte sich der Zusammenhang zwischen einem moderat bis schweren OSAS und erhöhter Gesamtmortalität in der australischen Busselton Sleep Cohort Study, in der ein HR von 6,2 (95\% Konfidenzintervall 2,0-19,4) ermittelt wurde (Marshall et al. 2008). Weitere Studien unterstützen den Zusammenhang zwischen einem schweren OSAS und einer insbesondere kardiovaskulär bedingten erhöhten Mortalität (Bliwise et al. 1988; Lavie et al. 2005; Muraja-Murro et al. 2013).

Eine aktuelle Meta-Analyse prospektiver Kohortenstudien kommt zu dem Schluss, dass nur das schwere, nicht jedoch das milde und moderate OSAS signifikant mit einem erhöhten Risiko (HR 1,6) für die Gesamtmortalität einhergeht (Pan et al. 2016).

Bei der Analyse der Sterblichkeitsrate muss berücksichtigt werden, dass das Mortalitätsrisiko vom Alter abhängig ist. Zudem existiert ein geschlechtsspezifischer Unterschied für das kardiovaskuläre Risiko. Aus diesen Gründen erfolgte die Durchführung einer Cox-Regressionsanalyse. Die um Alter und Geschlecht bereinigten Ergebnisse zeigten weiterhin eine positive Assoziation zwischen einem OSAS und erhöhten Mortalitätsrisiko (HR 1,78), jedoch war keine statistische Signifikanz mehr vorhanden. Nur in der Gruppe mit einem milden OSAS zeigte sich weiter ein signifikant erhöhtes Mortalitätsrisiko gegenüber den Studienteilnehmern ohne ein OSAS (HR 2,38, Konfidenzintervall 1,00-5,64, $\mathrm{p}=0,050)$.

Das lässt darauf schließen, dass der zuvor ermittelte signifikante Unterschied des Mortalitätsrisikos zwischen Patienten mit und ohne OSAS am ehesten auf die Variable Alter zurückzuführen ist.

Basierend auf den in der vorliegenden Studie erhobenen Daten hat das Vorhandensein eines ambulant diagnostizierten OSAS keinen signifikanten auf das Mortalitätsrisiko bei Patienten mit kardiovaskulären Risikofaktoren. Entgegen der Erwartung nimmt das 
Mortalitätsrisiko mit zunehmendem Schweregrad des OSAS ab.

Die erhobenen Daten unterstützend konnte in einer CPAP-Therapiestudie von McEvoy et al. (2016) gezeigt werden, dass die CPAP-Therapie bei Patienten mit einem moderaten und schweren OSAS keinen signifikanten Effekt auf die primären Endpunkte Tod, Myokardinfarkt, Apoplex oder kardiovaskuläre Hospitalisierung hat.

Bei der Interpretation der Ergebnisse müssen sowohl in der vorliegenden Studie als auch in vergleichbaren Publikationen die relativ geringe Effektgröße (Fallzahl an Verstorbenen) gemessen am großen Stichprobenumfang berücksichtigt werden (Marshall et al. 2008). Als Limitation der Studie kann dies zu Unsicherheiten bei der statistischen CoxRegressionsanalyse führen.

Wie im vorherigen Abschnitt beschrieben, wird die Vergleichbarkeit der einzelnen Studienergebnisse durch heterogene Studiendesigns limitiert (uneinheitliche Definitionen, unterschiedliche Einschlusskriterien bezüglich Alter, Geschlecht, Begleiterkrankungen, variierende Untersuchungstechniken zum Schlaf- und Atemmonitoring, Stichprobenumfang) (Punjabi 2008; Ross et al. 2000). Ein weiteres wesentliches Unterscheidungskriterium der vorliegenden Kohorte $\mathrm{zu}$ anderen Studienpopulationen ist die Art der Rekrutierung der Studienteilnehmer. In der vorliegenden Studie wurde ein zufällig ausgewähltes, bezüglich OSAS weitestgehend asymptomatisches Kollektiv mit vorhandenen kardiovaskulären Risikofaktoren untersucht. Die Patienten wurden aus einer laufenden Studie rekrutiert. Hieraus ergibt sich möglicherweise ein Bias aufgrund der vorbestehenden Grunderkrankungen.

Die Mehrzahl der Studien schlossen Teilnehmer ein, bei denen schon ein Schlafapnoesyndrom bekannt war, dieses teils schon behandelt wurde oder klinisch bereits der Verdacht auf eine SBAS (somit höhere Prätestwahrscheinlichkeit) gestellt wurde (Lee et al. 2013; Marin et al. 2005; Muraja-Murro et al. 2013; Ross et al. 2000). Ferner bestanden Unterschiede in der Beobachtungsdauer, die zwischen 5,1 Jahren (Lee et al. 2013) und 15,6 Jahren lag (Muraja-Murro et al. 2013).

Weiterhin ist zu berücksichtigen, dass SBAS keine akut verlaufenden Erkrankungen sind. Wie lange die Krankheit bereits unentdeckt vor Beginn der Datenerfassung (Baseline) bestand ist somit oftmals unklar, so dass Überlebenszeitangaben nicht zuverlässig bestimmt werden können.

Vergleichbar zu dieser Arbeit wurde in anderen publizierten Studien zur Mortalität mehrheitlich die Gesamtmortalität statt einer kardiovaskulären Mortalität angegeben. Die Erhebung der Todesursachen blieb oftmals durch verschiedene Faktoren unvollständig, so 
dass aufgrund einer fehlenden Aufschlüsselung erneut Ungenauigkeiten im Vergleich der spezifischen Mortalität resultierten. In vergleichbaren Studien zur Mortalität wurden als Todesursachen kardiovaskuläre Erkrankungen und Schlaganfälle mit jeweils weniger als 50 \% der Fälle angegeben (Lee et al. 2013; Punjabi et al. 2009; Young et al. 2008). Die in der vorliegenden Arbeit erhöhte Mortalität in der Gruppe der Patienten mit einem milden OSAS kann mutmaßlich auch in primär nicht kardiovaskulären Todesereignissen, wie etwa Tod aufgrund einer onkologischen Erkrankung oder Unfall, begründet sein.

\subsubsection{Geschlechtsspezifische Mortalität}

Eine weitere Forschungshypothese beschäftigte sich mit der geschlechtsspezifischen Mortalität. Obwohl laut Literatur deutlich häufiger Männer an einem OSAS erkranken (Young et al. 1993), war in der vorliegenden Studie die Verteilung der Geschlechter

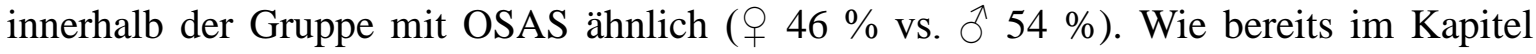
3.3.2 dargestellt, verstarben mehr Männer als Frauen. Die Hypothese, dass Männer aufgrund der in Kapitel 1.2.4 beschriebenen Ursachen und Risikofaktoren nicht nur eine höhere Prävalenz für ein OSAS, sondern auch eine höheres Mortalitätsrate aufweisen, konnte nicht belegt werden. Das männliche Geschlecht ist zwar tendenziell mit einem erhöhten Mortalitätsrisiko assoziiert, jedoch fand sich keine statistische Signifikanz. Entgegen der Annahme ging bei den Männern mit Zunahme des AHI der Anteil der Todesfälle zurück. Beim weiblichen Geschlecht konnte dieser Effekt nicht beobachtet werden. Die Aussagekraft wird allerdings durch die geringe Fallzahl an Ereignissen limitiert.

Erst nachdem eine Adjustierung auf das Alter erfolgte, konnte ein signifikanter Einfluss des Geschlechts auf die Mortalität nachgewiesen werden. Das weibliche Geschlecht wies erwartungsgemäß eine höhere Überlebenszeit auf.

Vergleichbare Literatur zur geschlechtsspezifischen Mortalität ist kaum vorhanden. Punjabi beschrieb, dass für Frauen in keiner Altersgruppe ein Zusammenhang zwischen einem OSAS und erhöhter Mortalität gezeigt werden konnte (Punjabi et al. 2009). In einer Studie von Marshall wurden verschiedene univariate Risikofaktoren für die Mortalität untersucht. Es wurde gezeigt, dass neben einem OSAS (AHI $\geq 15 / \mathrm{h}$ ) Diabetes, Alter und Rauchen die Mortalität signifikant beeinflussen. Übereinstimmend mit unseren Ergebnissen wies das männliche Geschlecht ein tendenziell höheres, statistisch jedoch nicht signifikantes Mortalitätsrisiko auf (Marshall et al. 2008). Die Arbeitsgruppe um Rich hingegen machte in einer univariaten Analyse das männliche Geschlecht als am stärksten 
mit der Gesamtmortalität assoziierte Variable noch vor Alter, BMI und AHI aus (Rich et al. 2012).

\subsubsection{Einfluss des Alters auf den AHI und die Mortalität}

Neben der Variable Geschlecht wurde auch der Einfluss des Alters auf die Schweregradausprägung des OSAS und insbesondere auf die Mortalität untersucht. Die Anzahl der Apnoen und Hypopnoen stieg entsprechend der Hypothese mit zunehmendem Alter signifikant an $(\mathrm{p}=0,005)$. Dieses ist kongruent mit den Daten anderer Studien (Bixler et al. 1998; Duran et al. 2001; Punjabi et al. 2009; Young et al. 2004).

Das Alter nahm auf die Mortalität der an OSAS erkrankten Patienten erwartungsgemäß einen signifikanten Einfluss. Dieses bestätigten auch weitere Studien (Rich et al. 2012). Interessanterweise zeigten die Ergebnisse weiterer Studien, dass Patienten mit OSAS im Alter < 50 Jahre das größte Mortalitätsrisiko haben und das Risiko mit höherem Alter abnimmt (Lavie et al. 2005; Rich et al. 2012). Punjabi beschrieb, dass insbesondere ein schweres OSAS bei Männern im Alter zwischen 40 und 70 Jahren mit einem erhöhten Mortalitätsrisiko assoziiert ist. Mit fortschreitendem Alter ist der Einfluss des OSAS auf die Mortalität schwieriger zu beurteilen, da die Wahrscheinlichkeit, an anderen Krankheiten zu versterben, steigt (Punjabi et al. 2009). Ähnlich wie Punjabi fand auch Lavie, dass mit Zunahme des Alters der Einfluss des OSAS auf die Mortalität nicht mehr vorhanden ist (Lavie et al. 2005). Wiederum bestätigten andere Studien einen Zusammenhang zwischen zunehmendem Alter und erhöhtem Mortalitätsrisiko (Pan et al. 2016).

\subsection{Einfluss des OSAS auf die Inzidenz von MACE}

Zahlreiche publizierte Studien zeigen, dass das OSAS einen unabhängigen Risikofaktor für das metabolische Syndrom sowie für kardio- und zerebrovaskuläre Erkrankungen wie arterielle Hypertonie, KHK, Herzinsuffizienz oder diastolische Dysfunktion darstellt (Bradley und Floras 2009; Fusetti et al. 2012; Peppard et al. 2000a; Shahar et al. 2001; Wachter et al. 2013). Hiervon ausgehend wurde die Hypothese formuliert, dass mit zunehmendem Schweregrad des OSAS ein häufigeres Auftreten kardiovaskulärer Ereignisse korreliert. In der vorliegenden Kohorte nahm jedoch entgegen der Forschungshypothese die Zahl der Ereignisse mit Zunahme des AHI ab. Die meisten MACE traten bei Patienten mit einem AHI $<5 / \mathrm{h}$ auf. 
Es ließ sich kein signifikanter Unterschied für das Auftreten von MACE zwischen den Studienteilnehmern ohne und mit OSAS feststellen (26\% vs. $22 \%$ ). Entgegen der Erwartung scheint jedoch das Vorhandensein eines OSAS (AHI > 5/h) tendenziell das Risiko für das Auftreten eines MACE zu reduzieren.

Nachdem ein Cox-Modell mit einer Adjustierung auf Geschlecht und Alter gerechnet worden ist, zeigt sich sogar eine statistische Signifikanz für den Zusammenhang zwischen einem OSAS und einem niedrigeren Risiko für das Auftreten von MACE. Das Vorhandensein eines OSAS scheint demnach einen protektiven Effekt für das Auftreten von MACE zu haben (HR 0,63, Konfidenzintervall 0,41-0,97, p=0,038). Eine weitere paradoxe Beobachtung war, dass mit Zunahme des OSAS-Schweregrades der protektive Effekt stärker ausfiel.

Dieses Ergebnis ist nicht plausibel mit dem zuvor beschriebenen erhöhten Mortalitätsrisiko für Patienten mit einem $\mathrm{AHI} \geq 5 / \mathrm{h}$. Als statistische Limitation ist dabei jedoch $\mathrm{zu}$ berücksichtigen, dass nur das Erstereignis eines MACE in die Analyse einging. Interessanterweise zeigte sich nach Berechnung der kumulativen Inzidenzen von MACE, dass nur die kardiovaskulären Hospitalisierungen signifikant häufiger in der Gruppe ohne OSAS auftraten $(\mathrm{p}=0,028)$. Hierdurch lässt sich vermutlich auch das insgesamt höhere Auftreten von MACE bei den Patienten mit einem AHI $<5 / \mathrm{h}$ erklären.

Eine mögliche Ursache für mehr kardiovaskuläre Hospitalisierungen in der Gruppe ohne OSAS könnte sein, dass diese Patienten bereits zum Zeitpunkt der Datenerhebung kränker waren und somit die Wahrscheinlichkeit für ein kardiovaskuläres Ereignis höher lag. In diesem Zusammenhang sei nochmals erwähnt, dass das Screeningkollektiv der vorliegenden Studie als Subpopulation aus einer laufenden Herzinsuffizienzstudie rekrutiert wurde. Im Vergleich der Baseline-Daten (vgl. Kapitel 3.2.2) spiegelt sich jedoch kein signifikanter Unterschied für die Begleiterkrankungen zwischen den Gruppen mit und ohne OSAS wider.

Als weitere Limitation ist zu diskutieren, dass kombinierte Endpunkte, wie in dieser Arbeit die MACE, Ereignisse von sehr verschiedener Wertigkeit zusammenfassen (z.B. Tod und kardiovaskuläre Hospitalisierung). Da insbesondere die kardiovaskulären Hospitalisierungen den Hauptteil der MACE bestimmen, ist es möglich, dass dadurch der Gesundheitszustand der Patienten nicht adäquat zum Ausdruck gebracht wird und somit die Patienten ohne OSAS kränker erscheinen.

Laut Recherche sind bislang kaum vergleichbare Daten verfügbar, da ähnliche Studien mehrheitlich den Zusammenhang eines OSAS und kardiovaskulären Erkrankungen einzig 
zum Zeitpunkt der initialen Datenerhebung analysieren.

Lediglich Marin et al. (2005) fanden im 10-Jahres-Follow-up, dass Patienten mit unbehandeltem schweren OSAS eine signifikant höhere Inzidenz für kardiovaskuläre Ereignisse und eine höhere kardiovaskuläre Mortalität als mit CPAP behandelte Patienten, unbehandelte Patienten mit milden oder moderaten OSAS sowie gesunde Probanden aufwiesen. Das Studienkollektiv war im Vergleich zu unseren Teilnehmern ausschließlich männlich, jünger und gesünder.

Wie im Kapitel 1.2.7 beschrieben, zeigten bereits mehrere klinische Beobachtungsstudien, dass durch die CPAP-Therapie neben einer Senkung des systolischen Blutdrucks, Verbesserung der Endothelfunktion und Steigerung der Insulinsensitivität, weniger kardiovaskuläre Komplikationen und Todesfälle aufgrund einer kardiovaskulären Genese zu beobachten sind (Meier und Andreas 2012). Laut Javaheri et al. (2017) sollte nach aktuellem wissenschaftlichen Stand die CPAP-Therapie bei Patienten mit arterieller Hypertonie und OSAS angewandt werden, um das Outcome zu verbessern. Wiederum scheint die CPAP-Therapie nur bei ausreichendem Gebrauch von mehr als vier Stunden pro Nacht einen positiven Einfluss auf kardio- und zerebrovaskuläre Erkrankungen zu haben (Javaheri et al. 2017). Hingegen beschrieben Naughton und Kee (2017), dass die CPAP-Therapie bei Patienten mit Herzinsuffizienz zwar kurzzeitig die kardiale Funktion verbessert, nicht jedoch das Überleben. Zu einem ähnlichen Ergebnis kamen auch McEvoy et al. (2016). Sie zeigten in einer aktuellen internationalen, multizentrischen Studie $(\mathrm{n}=2717)$, dass Patienten mit moderatem und schwerem OSAS sowie nachgewiesener kardiovaskulärer Erkrankung durch die CPAP-Therapie nicht vor dem Auftreten kardiovaskulärer Ereignisse geschützt werden (HR 1,1, 95 \% - Konfidenzintervall 0,911,32, $\mathrm{p}=0,34)$. Allerdings fanden sie, dass die CPAP-Therapie Symptome wie Tagesschläfrigkeit und Schnarchen signifikant reduziert sowie die Lebensqualität verbessert (McEvoy et al. 2016). Anhand dieser Ergebnisse sollte die Indikation zur komplexen CPAP-Therapie für spezielle Patientengruppen kritisch hinterfragt werden. Eine weitere Limitation dieser Studie ist, dass das Vorhandensein und der regelmäßige Gebrauch einer CPAP-Therapie nicht erhoben worden sind. 


\subsection{Evaluation eines prognostischen Parameters für die Mortalität}

\subsubsection{Einfluss der schlafbezogenen Hypoxie auf die Mortalität}

In der Mehrzahl der Studien wurden die Teilnehmer anhand des ermittelten AHI in Gruppen eingeteilt, denen dann ein unterschiedliches Mortalitätsrisiko zugeordnet werden konnte (Punjabi 2016). Es bleibt jedoch offen, ob der Parameter AHI alleinig für die pathophysiologischen Aspekte und klinischen Konsequenzen des OSAS sowie als therapeutische Zielgröße herangezogen werden kann (Hader et al. 2004; Pan et al. 2016). Laut Punjabi sind neben dem AHI weitere verschiedene Parameter notwendig, um die Komplexität der Erkrankung wiederzugeben (Punjabi 2016). Weiterhin ist fraglich, inwieweit der AHI als unabhängiger Prädiktor für eine erhöhte Mortalität gilt (Hader et al. 2004).

Wenige Studien haben bislang den Zusammenhang zwischen der Mortalität und anderen OSAS-relevanten Variablen untersucht und einen Einfluss dieser Variablen auf das Mortalitätsrisiko gefunden. Zu diesen Variablen zählen OSAS-typische Symptomatik, Schlafaufteilung, Sympathikusaktivität und hypoxische Ereignisse (Kendzerska et al. 2014; Pan et al. 2016). In der vorliegenden Studie wurde der Fokus auf die schlafbezogene Hypoxie gelegt.

Die periodisch auftretende Hypoxie ist zusammen mit der zentralnervösen Weckreaktion und den intrathorakalen Druckschwankungen als wesentlicher pathophysiologischer Mechanismus für die kardiovaskuläre Komorbidität verantwortlich (Meier und Andreas 2012; Quack et al. 2010). Die Hypoxie wird bei Patienten mit Schlafapnoe durch die nächtlichen Sauerstoffsättigungsabfälle bedingt. Um die Schwere der Hypoxie während des Schlafs zu quantifizieren, werden Parameter wie die mittlere Sauerstoffsättigung, minimale nächtliche Sauerstoffsättigung oder kumulative Entsättigungszeit herangezogen, die mittels Pulsoxymetrie ermittelt werden (Chaudhary et al. 1998; Punjabi 2008).

Ein weiterer Teil dieser Arbeit beschäftigte sich mit der Fragestellung, ob es abseits vom AHI in der Polygraphie messbare Parameter gibt, die mit einem erhöhten Mortalitätsrisiko korrelieren. Eine ähnliche Fragestellung bearbeiteten Punjabi et al. in der SHHS. Sie vermuteten, dass der Zusammenhang zwischen einer SBAS und Mortalität durch die Ausprägung der schlafbezogenen periodischen Hypoxämie vermittelt wird. Die Studie zeigte, dass der Grad der nächtlichen Hypoxämie als unabhängiger Prädiktor für die Mortalität gilt (Punjabi et al. 2009). Eine weitere Differenzierung der Hypoxämie wurde laut den publizierten Daten nicht vorgenommen. 


\subsubsection{Stellenwert der verschiedenen Parameter}

In der vorliegenden Arbeit wurden als Hypoxie-Indikatoren die vier Parameter kumulative Entsättigungszeit, mittlere und minimale Sauerstoffsättigung sowie der ODI näher betrachtet. Zunächst sollen die erhobenen Ergebnisse der Pulsoxymetrie in Bezug zu anderen Studien, die eine Polysomnographie respektive Polygraphie ausgewertet haben, gesetzt werden.

Dabei ist zu berücksichtigen, dass in den einzelnen Studien die Verteilung der Patienten mit einem milden, moderaten und schweren OSAS unterschiedlich ist. Ferner muss beachtet werden, dass untersuchungsbedingte und technische Messungenauigkeiten den Vergleich von Ergebnissen der Pulsoxymetrie einschränken.

In der vorliegenden Arbeit lag der Median für die minimale Sauerstoffsättigung des Screeningkollektivs bei $81 \%$ und für die mittlere Sauerstoffsättigung bei $94 \%$. Die Dauer der kumulativen Entsättigungszeit betrug 5 Minuten und der ODI 10,7/h im Median. Die Ergebnisse korrelieren weitestgehend mit den Daten anderer Studien. In einer von Yaggi et al. (2005) publizierten Studie ist in den Baseline-Charakteristika der Patienten mit OSAS sowie der Kontrollgruppe die minimale nächtliche arterielle Sauerstoffsättigung aufgeführt. Der Mittelwert beträgt 80,5 \% bei den OSAS-Patienten und 87,2 \% in der Kontrollgruppe und ist somit statistisch signifikant unterschiedlich (Yaggi et al. 2005). Leider wurde in der Studie kein Bezug auf die Mortalität genommen.

In einer weiteren Arbeit, die sich mit der Auswertung der Polysomnographie beschäftigte, wurden bei einem kleinen Kollektiv mit OSAS-Patienten mit einem AHI $\geq 15 / \mathrm{h}$ eine minimale Sauerstoffsättigung von $84,7 \%$ und eine mittlere Sauerstoffsättigung von $95 \%$ gefunden (Loube und Andrada 1999). In einer aktuelleren japanischen Studie, die zusätzlich noch in NREM- und REM-Schlaf unterschied, betrug die minimale Sättigung 83,6 \% bzw. 79,9 \% und die kumulative Entsättigungszeit 1,6 \% bzw. 11,5 \% (Hoshino et al. 2016). Die eingeschlossenen Patienten mit einem AHI $\geq 20 / \mathrm{h}$ hatten eine CPAPTherapie nach Diagnosestellung begonnen. In einer weiteren großen Kohortenstudie wurde eine mittlere Sauerstoffsättigung von 94,4 \% gemessen (Kendzerska et al. 2014).

Gottlieb et al. (2014) untersuchten 318 Patienten mit kardiovaskulären Risikofaktoren mittels ambulanter Polygraphie und ermittelten eine kumulative Entsättigungszeit $\mathrm{t} 90 \%$ von 5,2\% im Median.

Für eine hohe Reliabilität sollte ein Prädiktor eine möglichst geringe Variabilität gewährleisten. In diesem Zusammenhang gibt es eine Studie von Chaudhary et al. (1998). Die Arbeitsgruppe befasste sich nicht primär mit der Mortalitätsfrage, sondern suchte eine 
Messmethode mit der geringsten Variabilität während verschiedener Stunden des Polysomnographie-Monitorings. Sowohl die mittlere Sauerstoffsättigung als auch die kumulative Entsättigungszeit zeigten gegenüber der minimalen Sauerstoffsättigung eine geringere Variabilität. Zudem konnten sie zeigen, dass ein signifikanter Zusammenhang zwischen der mittleren Sauerstoffsättigung und der Schwere der Schlafapnoe besteht. Hinzugefügt werden muss jedoch, dass die Einteilung in die Schweregrade des OSAS nach anderen AHI-Grenzwerten erfolgte (Chaudhary et al. 1998). Auch anhand der vorliegenden Daten war zu beobachten, dass die mittlere Sauerstoffsättigung mit zunehmendem AHI tendenziell abnahm. ${ }^{13}$

In einer weiteren Studie von Hoshino et al. (2016) wird beschrieben, dass die kumulative Entsättigungszeit im Vergleich zur mittleren Sauerstoffsättigung die geeignetere Methode ist, die Schwere der Hypoxämie zu quantifizieren und zugleich die Zeit der Entsättigungen widerzuspiegeln .

\subsubsection{Einfluss der mittleren Sauerstoffsättigung und kumulativen Entsättigungszeit auf die Mortalität}

Eine Forschungshypothese ist, dass es weitere in der Polygraphie messbare Parameter gibt, die auf ein erhöhtes Mortalitätsrisiko schließen lassen und somit als therapeutische Zielgröße geeignet sind. Die vorliegende Arbeit beschäftigt sich insbesondere mit der Datenauswertung der genannten Parameter der pulsoxymetrischen Messung während des ambulanten Schlafscreenings.

Um einen statistischen Vergleich der erhobenen Daten zu ermöglichen, wurden die Messergebnisse des Screeningkollektivs nach Perzentilen eingeteilt (vgl. Kapitel 3.5).

Die Daten zeigten, dass für Patienten mit einer mittleren Sauerstoffsättigung von < $93 \%$ ein signifikant erhöhtes Mortalitätsrisiko besteht (17,2 \% vs. 7,9 \%, p=0,016). Nach Bereinigung der Statistik zeigte sich jedoch keine statistische Signifikanz mehr $(\mathrm{p}=0,166)$. Unter den Patienten, bei denen eine kumulative Entsättigungszeit t90\% von > 30 Minuten bestand, lag tendenziell eine erniedrigte Überlebenszeit vor, jedoch ohne Nachweis einer statistischen Signifikanz. Im Gegensatz dazu wurde für die Parameter ODI und minimale Sauerstoffsättigung kein Unterschied im Mortalitätsrisiko gefunden.

Vergleiche der vorliegenden Ergebnisse mit der Literatur sind nur eingeschränkt möglich, da bisher nur vereinzelt Ergebnisse der Polygraphie in ähnlicher Weise ausgewertet und in

\footnotetext{
${ }^{13}$ Die Ergebnisse der Screening-Polygraphie nach AHI der vorliegenden Studie sind ausführlich in der Arbeit von Dr. med. Daniela Klemmstein dargestellt (Klemmstein 2012).
} 
Verbindung zum Mortalitätsrisiko gesetzt worden sind.

Innerhalb einer Substudie der SHHS untersuchte die Arbeitsgruppe um Punjabi den Zusammenhang zwischen der Glukoseintoleranz und der nächtlichen Hypoxämie, wobei als Parameter die mittlere Sauerstoffsättigung und der Anteil an der Schlafzeit mit einer Sauerstoffsättigung $<90 \%$ betrachtet wurden. Vergleichbar mit unseren Ergebnissen zeigte sich ein signifikanter Einfluss der mittleren Sauerstoffsättigung. Eine mittlere Sauerstoffsättigung von $<93 \%$ korrelierte mit einer erhöhten Glukoseintoleranz (Punjabi et al. 2004).

In einer weiteren Studie wurde ermittelt, dass (nach Bereinigung von Störfaktoren wie Alter, Geschlecht, BMI, Rauchen, Komorbiditäten) der AHI keinen signifikanten Einfluss auf das kardiovaskuläre Outcome nahm. Hingegen korrelierte eine längere Schlafzeit mit einer Sauerstoffsättigung $<90 \%$ mit einem erhöhten Risiko für das Auftreten eines kardiovaskulären Ereignisses (Kendzerska et al. 2014).

Die mittels Polygraphie gemessene mittlere Sauerstoffsättigung und kumulative Entsättigungszeit sind Parameter mit geringer Variabilität. Zudem korreliert die mittlere Sauerstoffsättigung erwartungsgemäß mit der Schwere des OSAS. Die vorliegende Arbeit zeigte, dass diese beiden Parameter tendenziell eine Assoziation mit der Mortalität aufweisen. 


\section{Zusammenfassung}

Das obstruktive Schlafapnoesyndrom ist in den letzten Jahren zunehmend in den Fokus der Wissenschaft gerückt, da es mit einer gesteigerten kardio- und zerebrovaskulären Morbidität und Mortalität assoziiert ist. Aus verschiedensten Gründen bleibt ein OSAS jedoch häufig unterdiagnostiziert. Therapeutisch nimmt die CPAP-Therapie einen hohen Stellenwert ein, wenngleich aktuelle Studien keinen Einfluss auf das Überleben nachweisen konnten.

Das Ziel dieser Studie war, den Einfluss des OSAS auf die Langzeitprognose von Patienten mit kardiovaskulären Risikofaktoren zu ermitteln.

Im Rahmen einer prospektiven Kohortenstudie mit 1283 Patienten erfolgte bei 378 Teilnehmern zusätzlich ein Schlafapnoescreening mit Polygraphie, Bodyplethysmographie und standardisiertem Fragebogen. Das Screeningkollektiv war bezüglich eines OSAS weitestgehend asymptomatisch. Die Teilnehmer wurden anhand des ermittelten AHI vier Gruppen zugeordnet. Bei 140 Teilnehmern (37\%) wurde mit einem AHI $<5 / \mathrm{h}$ ein OSAS ausgeschlossen. Die Prävalenz für ein OSAS lag bei $63 \%$ (57\% der Frauen, $69 \%$ der Männer). Ein mildes OSAS (AHI 5-14/h) wurde bei $39 \%$ der Teilnehmer (n=149) festgestellt. Ein moderates OSAS wurde bei $16 \%(n=59)$ diagnostiziert, und bei $8 \%(n=30)$ der Patienten lag ein schweres OSAS (AHI > 30/h) vor. Patienten mit OSAS waren signifikant älter und häufiger männlich. Die Studienteilnehmer wurden über einen Zeitraum von 9 Jahren beobachtet.

Die Mortalität war in der Gruppe der Patienten mit OSAS im Vergleich zu Patienten ohne OSAS höher (12\% vs. $5 \%, \mathrm{p}=0,023$ ). Nach Adjustierung auf Geschlecht und Alter zeigte sich zwar ein moderater, aber statistisch nicht mehr signifikanter Zusammenhang mit einem erhöhten Mortalitätsrisiko (HR 1,78, 95 \% - Konfidenzintervall 0,76-4,14, p=0,181). Das Alter war erwartungsgemäß mit einer signifikant erhöhten Mortalität assoziiert (HR 1,11, $95 \%$ - Konfidenzintervall 1,06-1,16, p<0,001), während das weibliche Geschlecht mit einem tendenziell geringeren Mortalitätsrisiko einherging ( $\mathrm{HR}$ 0,59, $95 \%$ Konfidenzintervall 0,30-1,18, $\mathrm{p}=0,138$ ). Die höchste Gesamtmortalität wurde bei Patienten mit einem milden OSAS gefunden. Mit zunehmendem Schweregrad nahm das Mortalitätsrisiko tendenziell ab.

Im Vergleich der Patienten mit und ohne OSAS wurde kein statistisch signifikanter Unterschied für die Inzidenz eines MACE gefunden (22\% vs. $26 \%, \mathrm{p}=0,378)$. Nach Adjustierung auf Alter und Geschlecht korrelierte das OSAS sogar mit einem signifikant 
geringeren Risiko für das Auftreten von MACE (HR 0,63, $95 \%$ - Konfidenzintervall 0,410,97, $\mathrm{p}=0,038)$. Innerhalb der Patientengruppe mit OSAS zeigte sich, dass ein höherer Schweregrad nicht mit einem häufigeren Auftreten eines MACE korreliert.

Die vorliegende Arbeit konnte für Patienten mit Risikofaktoren für kardiovaskuläre Erkrankungen keinen signifikant negativen Effekt des OSAS auf die kardiovaskuläre Morbidität und Gesamtmortalität zeigen.

Von den untersuchten Einzelparametern des Schlafapnoescreenings zeigten die mittlere Sauerstoffsättigung (17\% vs. $8 \%, \mathrm{p}=0,166)$ und die kumulative Entsättigungszeit mit einer Sauerstoffsättigung < $90 \%(15 \%$ vs. $8 \%, p=0,057)$ tendenziell eine Assoziation mit der Mortalität. Weitere Studien müssen zeigen, ob diese Parameter als therapeutische Zielgrößen besser als klassische Parameter (z. B. AHI) geeignet sind. 


\section{Literaturverzeichnis}

American Academy of Sleep Medicine Task Force (1999): Sleep-related breathing disorders in adults: recommendations for syndrome definition and measurement techniques in clinical research. Sleep 22, 667-689

Andreas S: Obstructive sleep apnoea: clinical aspects/consequences. In: Simonds AK, de Backer W (Hrsg.): ERS Handbook respiratory sleep medicine. European Respiratory Society, Sheffield 2012, 32-38

Arias MA, Garcia-Rio F, Alonso-Fernandez A, Mediano O, Martinez I, Villamor J (2005): Obstructive sleep apnea syndrome affects left ventricular diastolic function: effects of nasal continuous positive airway pressure in men. Circulation 112, 375-383

Becker HF, Jerrentrup A, Ploch T, Grote L, Penzel T, Sullivan CE, Peter JH (2003): Effect of nasal continuous positive airway pressure treatment on blood pressure in patients with obstructive sleep apnea. Circulation $\underline{107}, 68-73$

Benjamin JA, Lewis KE (2008): Sleep-disordered breathing and cardiovascular disease. Postgrad Med J $\underline{84}, 15-22$

Bixler EO, Vgontzas AN, Ten Have T, Tyson K, Kales A (1998): Effects of age on sleep apnea in men: I. Prevalence and severity. Am J Respir Crit Care Med 157, 144-148

Bixler EO, Vgontzas AN, Lin HM, Ten Have T, Rein J, Vela-Bueno A, Kales A (2001): Prevalence of sleep-disordered breathing in women: effects of gender. Am J Respir Crit Care Med $\underline{163}$, 608-613

Bliwise DL, Bliwise NG, Partinen M, Pursley AM, Dement WC (1988): Sleep apnea and mortality in an aged cohort. Am J Public Health $\underline{78}, 544-547$

Bradley TD, Floras JS (2003): Sleep apnea and heart failure: Part I: obstructive sleep apnea. Circulation 107, 1671-1678

Bradley TD, Floras JS (2009): Obstructive sleep apnoea and its cardiovascular consequences. Lancet $\underline{373}$, 82-93

Brisco MA, Goldberg LR (2010): Sleep apnea in congestive heart failure. Curr Heart Fail Rep 7, 175-184

Carrera M, Barbe F, Sauleda J, Tomas M, Gomez C, Santos C, Agusti AG (2004): Effects of obesity upon genioglossus structure and function in obstructive sleep apnoea. Eur Respir J $\underline{23}, 425-429$

Chaudhary B, Dasti S, Park Y, Brown T, Davis H, Akhtar B (1998): Hour-to-hour variability of oxygen saturation in sleep apnea. Chest $\underline{113}, 719-722$ 
Chowdhury M, Adams S, Whellan DJ (2010): Sleep-disordered breathing and heart failure: focus on obstructive sleep apnea and treatment with continuous positive airway pressure. $\mathbf{J}$ Card Fail 16, 164-174

de Koning L, Merchant AT, Pogue J, Anand SS (2007): Waist circumference and waist-tohip ratio as predictors of cardiovascular events: meta-regression analysis of prospective studies. Eur Heart J $\underline{28}$, 850-856

Deegan PC, McNicholas WT (1995): Pathophysiology of obstructive sleep apnoea. Eur Respir J $\underline{8}, 1161-1178$

Douglas NJ, Polo O (1994): Pathogenesis of obstructive sleep apnoea/hypopnoea syndrome. Lancet $\underline{344}, 653-655$

Duran J, Esnaola S, Rubio R, Iztueta A (2001): Obstructive sleep apnea-hypopnea and related clinical features in a population-based sample of subjects aged 30 to $70 \mathrm{yr}$. Am $\mathrm{J}$ Respir Crit Care Med 163, 685-689

Eckert DJ, Malhotra A (2008): Pathophysiology of adult obstructive sleep apnea. Proc Am Thorac Soc 5, 144-153

Freedman N (2015): COUNTERPOINT: Does Laboratory Polysomnography Yield Better Outcomes Than Home Sleep Testing? No. Chest 148, 308-310

Fritscher LG, Mottin CC, Canani S, Chatkin JM (2007): Obesity and obstructive sleep apnea-hypopnea syndrome: the impact of bariatric surgery. Obes Surg 17, 95-99

Fung JW, Li TS, Choy DK, Yip GW, Ko FW, Sanderson JE, Hui DS (2002): Severe obstructive sleep apnea is associated with left ventricular diastolic dysfunction. Chest $\underline{121}$, 422-429

Fusetti M, Fioretti AB, Valenti M, Masedu F, Lauriello M, Pagliarella M (2012): Cardiovascular and metabolic comorbidities in patients with obstructive sleep apnoea syndrome. Acta Otorhinolaryngol Ital $\underline{32}$, 320-325

Golbin JM, Somers VK, Caples SM (2008): Obstructive sleep apnea, cardiovascular disease, and pulmonary hypertension. Proc Am Thorac Soc $\underline{5}$, 200-206

Gooneratne NS, Richards KC, Joffe M, Lam RW, Pack F, Staley B, Dinges DF, Pack AI (2011): Sleep disordered breathing with excessive daytime sleepiness is a risk factor for mortality in older adults. Sleep $\underline{34}, 435-442$

Gottlieb DJ, Punjabi NM, Mehra R, Patel SR, Quan SF, Babineau DC, Tracy RP, Rueschman M, Blumenthal RS, Lewis EF (2014): CPAP versus oxygen in obstructive sleep apnea. N Engl J Med 370, 2276-2285

Hader C, Sanner B, Rasche K (2004): Das obstruktive Schlafapnoe-Syndrom - Diagnostik. Dtsch Med Wochenschr 129, 566-569 
Hedner J, Bengtsson-Bostrom K, Peker Y, Grote L, Rastam L, Lindblad U (2006): Hypertension prevalence in obstructive sleep apnoea and sex: a population-based casecontrol study. Eur Respir J 27, 564-570

Hein H, Magnussen H (1998): Wie steht es um die medikamentöse Therapie bei schlafbezogenen Atmungsstörungen? Somnologie 2, 77-88

Herold G: Innere Medizin. Gerd Herold (Verlag), Köln 2016

Hoshino T, Sasanabe R, Murotani K, Arimoto M, Inagawa S, Tanigawa T, Uchida Y, Ogawa T, Ueda H, Shiomi T (2016): Polysomnographic parameters during non-rapid eye movement sleep predict continuous positive airway pressure adherence. Nagoya J Med Sci $\underline{78}, 195-203$

Javaheri S, Barbe F, Campos-Rodriguez F, Dempsey JA, Khayat R, Javaheri S, Malhotra A, Martinez-Garcia MA, Mehra R, Pack AI (2017): Sleep Apnea: Types, Mechanisms, and Clinical Cardiovascular Consequences. J Am Coll Cardiol $\underline{69}, 841-858$

Johns MW (1991): A new method for measuring daytime sleepiness: the Epworth sleepiness scale. Sleep $\underline{14}$, 540-545

Jordan AS, McSharry DG, Malhotra A (2014): Adult obstructive sleep apnoea. Lancet $\underline{383}$, 736-747

Kahwash R, Kikta D, Khayat R (2011): Recognition and management of sleep-disordered breathing in chronic heart failure. Curr Heart Fail Rep $\underline{8}, 72-79$

Kasai T (2012): Sleep apnea and heart failure. J Cardiol $\underline{60}, 78-85$

Kasai T, Bradley TD (2011): Obstructive sleep apnea and heart failure: pathophysiologic and therapeutic implications. J Am Coll Cardiol 57, 119-127

Kendzerska T, Gershon AS, Hawker G, Leung RS, Tomlinson G (2014): Obstructive sleep apnea and risk of cardiovascular events and all-cause mortality: a decade-long historical cohort study. PLoS Med 11, e1001599

Klemmstein D: Das obstruktive Schlafapnoesyndrom als Risikofaktor für eine diastolische Ventrikelfunktionsstörung. Med. Diss. Göttingen 2012

Laaban JP, Pascal-Sebaoun S, Bloch E, Orvoen-Frija E, Oppert JM, Huchon G (2002): Left ventricular systolic dysfunction in patients with obstructive sleep apnea syndrome. Chest $\underline{122}, 1133-1138$

Lam JC, Sharma SK, Lam B (2010): Obstructive sleep apnoea: definitions, epidemiology \& natural history. Indian J Med Res $\underline{131}, 165-170$

Lavie P, Lavie L, Herer P (2005): All-cause mortality in males with sleep apnoea syndrome: declining mortality rates with age. Eur Respir J 25, 514-520

Lee JE, Lee CH, Lee SJ, Ryu Y, Lee WH, Yoon IY, Rhee CS, Kim JW (2013): Mortality of patients with obstructive sleep apnea in Korea. J Clin Sleep Med 9, 997-1002 
Leitlinie 2015 zur HNO-spezifischen Therapie der obstruktiven Schlafapnoe bei Erwachsenen. http://www.awmf.org/leitlinien/detail/11/017-069.html; Zugriff am 02.04.2017

Loube DI, Andrada TF (1999): Comparison of respiratory polysomnographic parameters in matched cohorts of upper airway resistance and obstructive sleep apnea syndrome patients. Chest $\underline{115}, 1519-1524$

Lüthje L, Andreas S (2008): Obstructive sleep apnea and coronary artery disease. Sleep Med Rev $\underline{12}, 19-31$

Malhotra A, Orr JE, Owens RL (2015): On the cutting edge of obstructive sleep apnoea: where next? Lancet Respir Med $\underline{3}, 397-403$

Mannarino MR, Di Filippo F, Pirro M (2012): Obstructive sleep apnea syndrome. Eur J Intern Med 23, 586-593

Marin JM, Carrizo SJ, Vicente E, Agusti AG (2005): Long-term cardiovascular outcomes in men with obstructive sleep apnoea-hypopnoea with or without treatment with continuous positive airway pressure: an observational study. Lancet $\underline{365}, 1046-1053$

Marshall NS, Wong KK, Liu PY, Cullen SR, Knuiman MW, Grunstein RR (2008): Sleep apnea as an independent risk factor for all-cause mortality: the Busselton Health Study. Sleep $\underline{31}, 1079-1085$

Mason M, Welsh EJ, Smith I (2013): Drug therapy for obstructive sleep apnoea in adults. Cochrane Database Syst Rev, CD003002

McEvoy RD, Antic NA, Heeley E, Luo Y, Ou Q, Zhang X, Mediano O, Chen R, Drager LF, Liu Z (2016): CPAP for Prevention of Cardiovascular Events in Obstructive Sleep Apnea. N Engl J Med 375, 919-931

McNicholas WT (2008): Diagnosis of obstructive sleep apnea in adults. Proc Am Thorac Soc $\underline{5}, 154-160$

McNicholas WT, Bonsigore MR, Management Committee of ECAB (2007): Sleep apnoea as an independent risk factor for cardiovascular disease: current evidence, basic mechanisms and research priorities. Eur Respir J 29, 156-178

Meier M, Andreas S (2012): Mechanismen der kardiovaskulären Komorbidität bei Patienten mit obstruktivem Schlafapnoesyndrom. Pneumologie 66, 650-657

Morimoto S, Takahashi T, Okaishi K, Okuro M, Nakahashi T, Sakamoto D, Mizuno T, Kanda T, Takahashi M, Toga H (2012): Sleep apnoea syndrome as a risk for mortality in elderly inpatients. J Int Med Res $\underline{40}$, 601-611

Muraja-Murro A, Eskola K, Kolari T, Tiihonen P, Hukkanen T, Tuomilehto H, Peltonen M, Mervaala E, Toyras J (2013): Mortality in middle-aged men with obstructive sleep apnea in Finland. Sleep Breath 17, 1047-1053 
Naughton MT, Kee K (2017): Sleep apnoea in heart failure: To treat or not to treat? Respirology 22, 217-229

Nieto FJ, Young TB, Lind BK, Shahar E, Samet JM, Redline S, D'Agostino RB, Newman AB, Lebowitz MD, Pickering TG (2000): Association of sleep-disordered breathing, sleep apnea, and hypertension in a large community-based study. Sleep Heart Health Study. JAMA $\underline{283}, 1829-1836$

Nieto FJ, Peppard PE, Young T, Finn L, Hla KM, Farre R (2012): Sleep-disordered breathing and cancer mortality: results from the Wisconsin Sleep Cohort Study. Am J Respir Crit Care Med 186, 190-194

O'Connor GT, Caffo B, Newman AB, Quan SF, Rapoport DM, Redline S, Resnick HE, Samet J, Shahar E (2009): Prospective study of sleep-disordered breathing and hypertension: the Sleep Heart Health Study. Am J Respir Crit Care Med 179, 1159-1164

Oliveira W, Campos O, Cintra F, Matos L, Vieira ML, Rollim B, Fujita L, Tufik S, Poyares D (2009): Impact of continuous positive airway pressure treatment on left atrial volume and function in patients with obstructive sleep apnoea assessed by real-time threedimensional echocardiography. Heart $\underline{95}$, 1872-1878

Pack AI, Maislin G (2001): Who should get treated for sleep apnea? Ann Intern Med 134, 1065-1067

Pan L, Xie X, Liu D, Ren D, Guo Y (2016): Obstructive sleep apnoea and risks of all-cause mortality: preliminary evidence from prospective cohort studies. Sleep Breath $\underline{20}, 345-353$

Peled N, Abinader EG, Pillar G, Sharif D, Lavie P (1999): Nocturnal ischemic events in patients with obstructive sleep apnea syndrome and ischemic heart disease: effects of continuous positive air pressure treatment. J Am Coll Cardiol 34, 1744-1749

Peppard PE, Young T, Palta M, Skatrud J (2000a): Prospective study of the association between sleep-disordered breathing and hypertension. N Engl J Med 342, 1378-1384

Peppard PE, Young T, Palta M, Dempsey J, Skatrud J (2000b): Longitudinal study of moderate weight change and sleep-disordered breathing. JAMA 284, 3015-3021

Peppard PE, Young T, Barnet JH, Palta M, Hagen EW, Hla KM (2013): Increased prevalence of sleep-disordered breathing in adults. Am J Epidemiol 177, 1006-1014

Polo O, Berthon-Jones M, Douglas NJ, Sullivan CE (1994): Management of obstructive sleep apnoea/hypopnoea syndrome. Lancet $\underline{344}$, 656-660

Punjabi NM, Shahar E, Redline S, Gottlieb DJ, Givelber R, Resnick HE, Sleep Heart Health Study I (2004): Sleep-disordered breathing, glucose intolerance, and insulin resistance: the Sleep Heart Health Study. Am J Epidemiol 160, 521-530

Punjabi NM (2008): The epidemiology of adult obstructive sleep apnea. Proc Am Thorac Soc $\underline{5}, 136-143$ 
Punjabi NM, Caffo BS, Goodwin JL, Gottlieb DJ, Newman AB, O'Connor GT, Rapoport DM, Redline S, Resnick HE, Robbins JA (2009): Sleep-disordered breathing and mortality: a prospective cohort study. PLoS Med $\underline{6}$, e1000132

Punjabi NM (2016): COUNTERPOINT: Is the Apnea-Hypopnea Index the Best Way to Quantify the Severity of Sleep-Disordered Breathing? No. Chest 149, 16-19

Quack I, Fritz A, Rump LC (2010): Schlafapnoesyndrom bei chronischer Niereninsuffizienz. Nephrologe $\underline{5}, 43-48$

Quan SF, Howard BV, Iber C, Kiley JP, Nieto FJ, O'Connor GT, Rapoport DM, Redline S, Robbins J, Samet JM (1997): The Sleep Heart Health Study: design, rationale, and methods. Sleep 20, 1077-1085

Rasekaba T, Lee AL, Naughton MT, Williams TJ, Holland AE (2009): The six-minute walk test: a useful metric for the cardiopulmonary patient. Intern Med J $\underline{39}, 495-501$

Redline S (2017): Screening for Obstructive Sleep Apnea: Implications for the Sleep Health of the Population. JAMA $\underline{317}$, 368-370

Redline S, Yenokyan G, Gottlieb DJ, Shahar E, O'Connor GT, Resnick HE, Diener-West M, Sanders MH, Wolf PA, Geraghty EM (2010): Obstructive sleep apnea-hypopnea and incident stroke: the sleep heart health study. Am J Respir Crit Care Med 182, 269-277

https://www.resmedshop.de/themenwelt/schlafapnoe; Zugriff am 01.06.2017

Rich J, Raviv A, Raviv N, Brietzke SE (2012): All-cause mortality and obstructive sleep apnea severity revisited. Otolaryngol Head Neck Surg 147, 583-587

Rodenbeck A (2013): Manual der American Academy of Sleep Medicine. Überblick über die Updateversion 2.0. Somnologie 17, 122-130

Ross SD, Sheinhait IA, Harrison KJ, Kvasz M, Connelly JE, Shea SA, Allen IE (2000): Systematic review and meta-analysis of the literature regarding the diagnosis of sleep apnea. Sleep $\underline{23}, 519-532$

S3-Leitlinie 2009: Nicht erholsamer Schlaf/Schlafstörungen. Deutsche Gesellschaft für Schlafforschung und Schlafmedizin (DGSM). Somnologie 13, 4-160

Sanner B, Hader C, Rasche K (2004): Das obstruktive Schlafapnoe-Syndrom (OSAS) Therapie. Dtsch Med Wochenschr 129, 570-576

Schulz R, Olschewski H, Grimminger F, Seeger W (2000): Prävalenz von Schlaganfall und transitorischer ischämischer Attacke (TIA) bei obstruktiver Schlaf-Apnoe: eine retrospektive Erhebung an 187 konsekutiven Patienten. Pneumologie 54, 575-579

Schulz R, Grebe M, Eisele HJ, Mayer K, Weissmann N, Seeger W (2006): Vaskuläre Erkrankungen bei obstruktiver Schlafapnoe. Med Klin 101, 321-327

Schulz R, Blau A, Borgel J, Duchna HW, Fietze I, Koper I, Prenzel R, Schadlich S, Schmitt J, Tasci S, et al. (2007): Sleep apnoea in heart failure. Eur Respir J 29, 1201-1205 
Schwartz AR, Patil SP, Laffan AM, Polotsky V, Schneider H, Smith PL (2008): Obesity and obstructive sleep apnea: pathogenic mechanisms and therapeutic approaches. Proc Am Thorac Soc $\underline{5}, 185-192$

Shahar E, Whitney CW, Redline S, Lee ET, Newman AB, Nieto FJ, O'Connor GT, Boland LL, Schwartz JE, Samet JM (2001): Sleep-disordered breathing and cardiovascular disease: cross-sectional results of the Sleep Heart Health Study. Am J Respir Crit Care Med 163, $19-25$

Shamsuzzaman AS, Gersh BJ, Somers VK (2003): Obstructive sleep apnea: implications for cardiac and vascular disease. JAMA $\underline{290}, 1906-1914$

Siegenthaler W, Blum HE: Klinische Pathophysiologie. Thieme Verlag, Stuttgart 2006

Siegrist J, Peter J, Himmelmann J, Geyer S (1987): Erfahrungen mit einem Anamnesebogen zur Diagnostik der Schlafapnoe. Prax Klin Pneumol 41, 357-363

Sommer UJ, Heiser C, Gahleitner C, Herr RM, Hormann K, Maurer JT, Stuck BA (2016): Tonsillectomy with Uvulopalatopharyngoplasty in Obstructive Sleep Apnea. Dtsch Arztebl Int $\underline{113}, 1-8$

Steiner S, Schwartzkopff B, Strauer BE, Schultze-Werninghaus G, Duchna HW (2005): Schlafapnoe: Bedeutung bei Patienten mit Herzinsuffizienz. Dtsch Med Wochenschr 130, 468-472

Storre JH (2016): Wann Überweisung in ein Schlaflabor? Dtsch Arztebl Int $\underline{113}, 14-17$

Strollo PJ, Jr., Rogers RM (1996): Obstructive sleep apnea. N Engl J Med 334, 99-104

Stuck BA, Maurer JT: Schlafbezogene Atmungsstörungen. Springer Medizin Verlag, Berlin Heidelberg 2013

Tuomilehto H, Seppa J, Uusitupa M (2013): Obesity and obstructive sleep apnea--clinical significance of weight loss. Sleep Med Rev 17, 321-329

Varol E, Akcay S, Ozaydin M, Ozturk O, Cerci SS, Sahin U (2010): Influence of obstructive sleep apnea on left ventricular mass and global function: sleep apnea and myocardial performance index. Heart Vessels $\underline{25}$, 400-404

Wachter R, Luthje L, Klemmstein D, Luers C, Stahrenberg R, Edelmann F, Holzendorf V, Hasenfuss G, Andreas S, Pieske B (2013): Impact of obstructive sleep apnoea on diastolic function. Eur Respir J 41, 376-383

Yaggi HK, Concato J, Kernan WN, Lichtman JH, Brass LM, Mohsenin V (2005): Obstructive sleep apnea as a risk factor for stroke and death. N Engl J Med 353, 2034-2041

Young T, Palta M, Dempsey J, Skatrud J, Weber S, Badr S (1993): The occurrence of sleep-disordered breathing among middle-aged adults. N Engl J Med $\underline{328}$, 1230-1235

Young T, Peppard PE, Gottlieb DJ (2002a): Epidemiology of obstructive sleep apnea: a population health perspective. Am J Respir Crit Care Med 165, 1217-1239 
Young T, Shahar E, Nieto FJ, Redline S, Newman AB, Gottlieb DJ, Walsleben JA, Finn L, Enright P, Samet JM (2002b): Predictors of sleep-disordered breathing in communitydwelling adults: the Sleep Heart Health Study. Arch Intern Med 162, 893-900

Young T, Skatrud J, Peppard PE (2004): Risk factors for obstructive sleep apnea in adults. JAMA $291,2013-2016$

Young T, Finn L, Peppard PE, Szklo-Coxe M, Austin D, Nieto FJ, Stubbs R, Hla KM (2008): Sleep disordered breathing and mortality: eighteen-year follow-up of the Wisconsin sleep cohort. Sleep $\underline{31}$, 1071-1078

Zwiener I, Blettner M, Hommel G (2011): Überlebenszeitanalyse. Dtsch Arztebl Int 108, 163-169 


\section{Danksagung}

Mein besonderer Dank gilt meinem Betreuer Prof. Dr. med. Rolf Wachter für die kompetente und geduldige Betreuung sowie die hilfreiche und konstruktive Unterstützung bei der Fertigstellung der Dissertation.

Weiterhin bedanke ich mich bei Herrn Dr. rer. nat. Meinhard Mende vom Zentrum für Klinische Studien Leipzig - Koordinationszentrum für Klinische Studien, Universität Leipzig für die Beratung und Hilfestellung bei der statistischen Auswertung.

Für die gute Kooperation und Hilfestellung bei der Patientenrekrutierung und der Erfassung der klinischen Daten danke ich den wissenschaftlichen Mitarbeiterinnen und Mitarbeitern der kardiologischen Studienambulanz der Universitätsmedizin Göttingen.

Mein weiterer Dank gilt meinem Ehemann Dr. med. Philipp Koch, der mich bei der Fertigstellung dieser Arbeit jederzeit motiviert und unterstützt hat. 